\title{
Otimização em \\ Sistemas Distribuídos com \\ Partição de Servidores
}

\section{Rosalina de Carvalho Fabbri}

\author{
DISSERTAÇÃO APRESENTADA \\ $\mathrm{AO}$ \\ INSTITUTO DE MATEMÁTICA E ESTATÍSTICA \\ DA \\ UNIVERSIDADE DE SÃO PAULO \\ PARA OBTENÇÃO DO GRAU DE MESTRE \\ EM \\ MATEMÁTICA APLICADA
}

Área de Concentração: Ciência de Computação

Orientador: Prof. Dr. Carlos Humes Júnior

-SÃO PAULO, Fevereiro de $1989^{-}$ 
"O homem se torna muitas vezes

o que ele proprio acredita que é.

Se eu insisto em repetir para mim mesmo

que não posso fazer uma determinada coisa, é possivel que eu acabe me tornando

realmente incapaz de fazê-la.

Ao contrário,

se tenho a convicção de que posso fazê-la,

certamente adquirirei

a capacidade de realizá-la,

mesmo que não a tenha no começo."

Gandhi

A meu pai

Alfredo de Carvalho 


\section{Agradecimentos}

Ao Prof. Carlos Humes Júnior,

por todo incentivo e apoio para a realização desse trabalho, além da sua contribuição como orientador e amigo.

À Ana Flora, que tão bem me recebeu em todas as vezes em que trabalhei em sua casa.

Ao Prof. Carlos A.Dantas, pelas discussões e palpites proveitosos.

Aos funcionários do IME-USP que de alguma forma participaram do trabalho.

À minha família, principalmente à minha mãe, pelo apoio incondicional e por estar sempre tão perto.

Em especial, à Eugênio, por tudo que nem precisa ser dito. 


\section{PREFÁCIO}

A motivação desse trabalho é o estudo de sistemas distribuídos, principalmente do ponto de vista de análise de desempenho.

O capítulo 2 é dedicado a apresentação conceitual de sistemas distribuídos. Neste capítulo são considerados vários fatores que influem no desempenho de um sistema distribuído, entre eles, destacamos a escolha da estratégia de alocação de recursos do sistema ('load sharing'). Destaque este que nos levou à apresentação de algumas dessas estratégias com mais detalhes.

Os capítulos seguintes dedicam-se ao estudo da estratégia estática denominada 'partição dos servidores', que verificou-se ser bastante sensível a variação da distribuição da carga.

No capítulo 3 a estratégia é apresentada formalmente e são feitas análises voltadas a projeto ótimo $((\lambda, \mu)$-problema $)$, a ajuste de desempenho ( $\lambda$-problema) e a planejamento de evolução ( $\mu$-problema) do sistema distribuído com partições singulares.

No capítulo 4 pretende-se estender a análise, considerando particulares grupos não singulares da partição.

A conclusão apresenta alguns dos resultados obtidos no desenrolar deste trabalho, em particular a questão dos níveis de desativação e a comparação parcial entre processamento centralizado e distribuído. São também apresentadas possibilidades de pesquisa futura com breves referências a trabalhos relevantes em linhas com maior ou menor similaridade. 


\section{Capítulo 1}

\section{Introdução}

É fato que as interconexões de processadores, formando os chamados sistemas distribuídos, tem sido de grande interesse, não somente por serem teoricamente viáveis, mas também por serem funcional e economicamente atrativas.

A funcionalidade desses sistemas surge da melhora do desempenho global, obtida através do compartilhamento dos recursos (tais como bases de dados, 'softwares', periféricos, CPU etc.) e da distribuição da carga da rede, sendo que esta distribuição consiste em mover jobs (elementos de carga) para execução em processadores menos carregados.

Para que as vantagens resultantes da interconexão sejam obtidas são necessárias decisões a nivel de projeto. Tem-se utilizado teoria das filas como ferramenta efetiva para o estudo e modelagem de sistemas de computadores. Caracterizou-se então a junção do interesse do estudo de projeto de sistemas distribuídos e do interesse na área de otimização, mais precisamente, na área de programação matemática.

A pesquisa da literatura, nessa área, nos levou ao excelente trabalho de Y.T.Wang ([29]), que é apresentado neste trabalho com mais formalismo devido ao seu grande valor intrínseco. Passamos então a questionar a validade da medida de desempenho apresentada, que utiliza a pior condição de carga sobre o pior projeto possível como paradigma de comparação. Mais ainda, passamos a questionar processamento distribuído versus pro- 
cessamento centralizado, que é um assunto já brevemente tratado por Kleinrock (em [16]) e Humes (em [14]), no caso de sistemas de filas $M / M / K$ homogêneos.

Assim, nosso objetivo passou a ser o estudo de sistemas distribuídos não homogêneos e a discussão da questão da centralização versus descentralização de sistemas. Consideramos para tanto uma classe de sistemas distribuídos, escolhendo uma dentre as estratégias de alocação de recursos apresentadas, a estratégia de 'partição dos servidores'.

Para essa estratégia escolhida, estudamos otimalidade para três problemas definidos: o problema do projeto do sistema distribuído associado, chamado $(\lambda, \mu)$-problema, o problema do ajuste de desempenho, chamado $\lambda$-problema, que surge devido ao aumento da demanda de processamento e o problema da evolução, chamado $\mu$-problema, que surge da impossibilidade da melhora de desempenho do sistema atual devido a um aumento excessivo de demanda. Esse estudo considerou partições onde os jobs que entram por uma origem são todos atendidos por um único processador (servidor) associado, as chamadas partições singulares. A partir desse estudo pode-se comprovar que o balanceamento de carga, que consiste da divisão da carga igualmente entre os servidores, em geral não é uma boa decisão e ainda que sob algumas hipóteses, principalmente a de linearidade entre custos e capacidades de atendimento dos servidores, a centralização pode ser a melhor escolha.

O próximo passo foi o estudo de partições não singulares e não homogêneas, correspondentes a sistemas $M / M / K(K \geq 2)$ não homogêneos, com o intuito de comprovar o fato de que a centralização de atendimento leva ao melhor desempenho e que a homogeneidade dos servidores, tão usual na literatura, nunca o faz. Era nosso interesse, nesse caso, exibir as expressões gerais que descrevem os sistemas $M / M / K$ não homogêneos, mas infelizmente conseguimos apenas para o caso $K=2$ e algumas expressões de difícil manuseio e que foram testadas numericamente para o caso $K=3$, o que nos levou apenas à conjectura dos resultados acima descritos.

Finalizamos com a apresentação de várias possíveis linhas de pesquisa em aberto, sendo que algumas são similares e outras têm enfoques distintos da linha apresentada neste trabalho. 


\section{Capítulo 2}

\section{Análise de Desempenho de Sistemas Distribuídos}

Tem sido amplamente difundida a idéia da utilização de sistemas de computadores distribuídos, que são na verdade microprocessadores interconectados e separados por distâncias curtas, usualmente consideradas como de no máximo dois quilômetros.

Estes sistemas são de baixo custo e têm bom desempenho ('performance') pois fornecem a possibilidade de utilização global dos recursos de processamento e ainda a possibilidade de compartilhamento de informações entre os vários usuários do sistema.

No entanto, o desempenho de um sistema distribuido é afetado diretamente pelos parâmetros que o definem, ou seja, pelo seu projeto ('design'). Entre esses parâmetros, temos a carga do sistema, ou seja, a necessidade de processamento, que usualmente é um dado para o projeto e parâmetros que caracterizam o projeto, tais como: o número de microprocessadores no sistema, a capacidade de processamento do sistema, a distribuição da carga entre os microprocessadores e a capacidade de processamento de cada microprocessador.

Este capítulo tem por objetivo o estudo dos sistemas distribuídos e de como o projeto de um sistema distribuído afeta o seu desempenho global.

Existem vários outros parâmetros importantes do projeto, como o procedimento para roteamento da carga no sistema, a disciplina de atendimento do sistema (prioridade), as 
estratégias de bufferização (pois cada elemento de carga precisa esperar pelo atendimento), controle de erros etc., sendo que estes não serão considerados para simplicidade da análise.

A definição de sistema distribuído local apresentada neste capítulo é baseada no trabalho apresentado por Y.T. Wang, em [29], que motivou inicialmente o nosso estudo.

\subsection{Apresentação de sistemas distribuídos}

Seguindo [29], utilizaremos as seguintes definições e convenções:

DEFINIÇÃO: Um sistema distribuído consiste de um conjunto de microprocessadores interconectados por um meio de comunicação. O sistema distribúido será modelado por um grafo, onde os nós são os microprocessadores e as arestas são o meio de comunicação.

DEFINIÇÃO: Origens são os nós através dos quais as demandas de processamento ('jobs') entram no sistema. O termo job vai ser utilizado para denotar uma seqüência de tarefas a serem executadas por um processador.

DEFINIÇÃO: Servidores são os nós que processam os jobs. Um mesmo nó pode ser origem e servidor simultaneamente.

A fim de podermos avaliar o projeto de um sistema distribuído, introduzimos uma medida de desempenho. Esta pode ser dada pelo tempo médio de um job no sistema ('delay') e/ou pela vazão do sistema ('throughput'), que é o número de jobs que saem do sistema por unidade de tempo. Desta forma, o desempenho ótimo do sistema seria aquele associado a um mínimo tempo médio e/ou a uma máxima vazão.

Poder-se-ia ainda avaliar a adequação de um projeto verificando-se a utilização média dos recursos do sistema. Por exemplo, poder-se-ia verificar se os servidores estão sendo bem utilizados, ou se estão em geral saturados, ou mesmo se estão subutilizados ('idle').

Vamos então considerar o seguinte modelo para o estudo de sistemas distribuídos: 


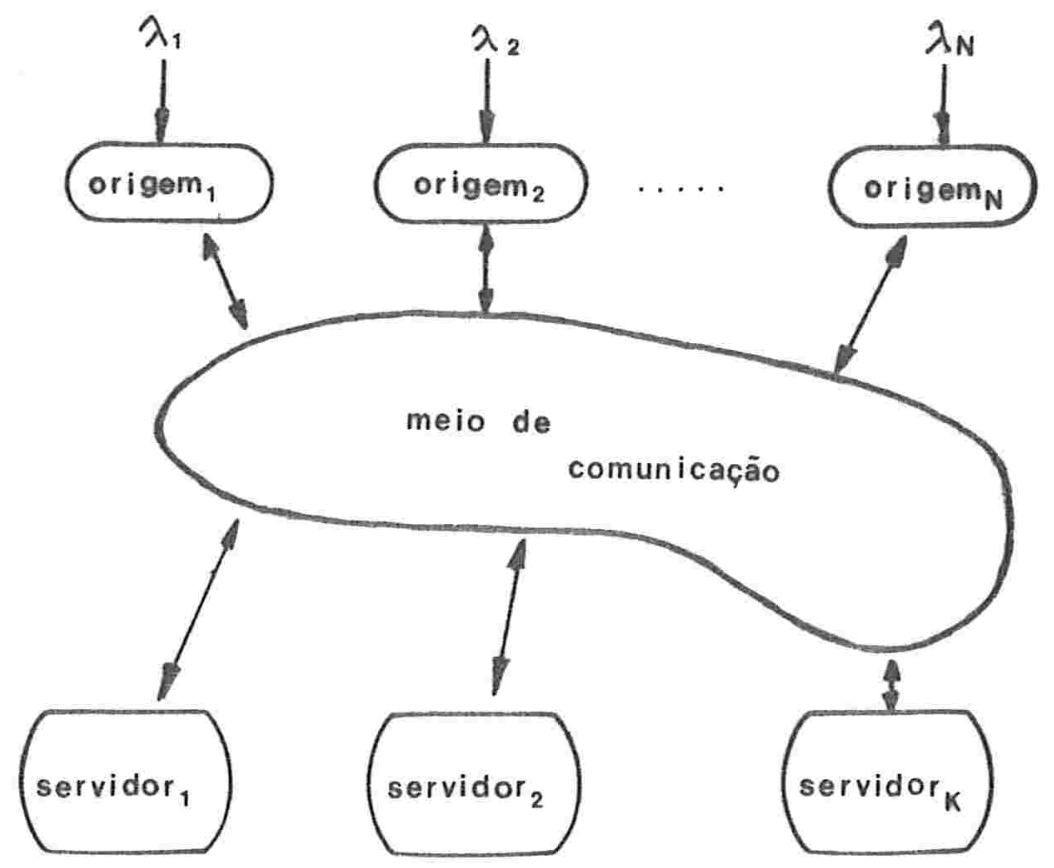

onde usaremos ao longo do trabalho as notações reservadas:

$\mathrm{N}$ - número de nós origem

K - número de nós servidores

$\lambda_{i}$ - taxa média de entrada de jobs no sistema, via o i-ésimo nó origem, $i=1,2, \cdots, N$.

Note-se que esta notação corresponde a uma hipótese de homogeneidade dos jobs.

No texto, podemos nos referir a $\lambda_{i}$ por carga na origem $i$.

Com isto introduzimos uma aleatoriedade na demanda de serviço no sistema através da variável $\lambda_{i}$, que consiste na aleatoriedade de chegadas dos jobs no sistema.

A cada nó servidor está associada uma capacidade de atendimento. Alguns conceitos se fazem necessários para introduzir a notação para a capacidade de atendimento.

Inicialmente vamos considerar que um job pode ser descrito por um conjunto de operações padrão a serem executadas. Esta consideração pode ser entendida da seguinte forma: a todo job corresponde um 'código objeto' que vem a ser uma série de operações, que podem ser consideradas semelhantes em termos de tempo médio de processamento e que chamamos de operações padrão. Dizemos então que o comprimento médio de um job é o número de instruções padrão a que ele corresponde. Suporemos que os jobs que entram 
no sistema têm comprimentos independentes, que são distribu!dos exponencialmente com média $\ell$.

Por outro lado, a cada servidor está associada uma capacidade de processamento, que é dada pelo número de instruções padrão que o servidor pode executar por segundo. Seja $C_{j}$, a capacidade de processamento do servidor $j(j=1,2, \cdots, K)$.

Temos então que o tempo de atendimento de um job no servidor $\mathrm{j}$ é distribúdo exponencialmente com média $\mu_{j}^{-1}$, sendo que

$$
\mu_{j}^{-1}=\frac{\text { comprimento médio de um job }}{\text { capacidade de processamento do servidor } \mathrm{j}}=\frac{\ell}{C_{j}} \quad j=1,2, \cdots, K
$$

Note-se que $\mu_{j}$ corresponde à taxa média de atendimento no servidor $\mathrm{j}$. No texto, podemos nos referir a $\mu_{j}$ por capacidade de atendimento do servidor $j$.

Nestas condições, o caráter aleatório do atendimento é dado pelo tempo de atendimento de um job e tem distribuição exponencial de forma que o servidor tem uma dada capacidade de processamento e os comprimentos dos jobs são distribuídos exponencialmente.

Podemos ainda definir os seguintes parâmetros globais para o sistema:

$\lambda$ - carga total do sistema

$$
\lambda \triangleq \sum_{i=1}^{N} \lambda_{i}
$$

$\mu$ - capacidade total de atendimento do sistema

$$
\mu \triangleq \sum_{j=1}^{K} \mu_{j}
$$

$\rho$ - fator de utilização global do sistema

$$
\rho \triangleq \frac{\lambda}{\mu}
$$

Para facilitar a apresentação, introduzimos a seguinte notação:

$S(\lambda, \mu, N, K)$ denota um sistema distribuído com $\mathrm{N}$ origens e $\mathrm{K}$ servidores, com carga total $\lambda$ e capacidade total de atendimento $\mu$. Obviamente esta notação não descreve peculiaridades locais, isto é, a distribuição da carga nas origens e a alocação de capacidades nos servidores, que são os valores $\lambda_{i}$ e $\mu_{j}$ conforme definidos anteriormente.

Assim, para o sistema $S(\lambda, \mu, N, K)$ apenas os valores $\lambda, \mu, \mathrm{N}, \mathrm{K}$ são pré-determinados, ficando a cargo do texto explicitar outros dados associados ao sistema que sejam conhecidos. 
Ao longo do texto utilizamos para descrever uma fila a notação $A / B / m$, que denota uma fila para $m$ servidores, onde

A - distribuição dos tempos entre chegadas

B - distribuição dos tempos de serviço

Em geral, usamos $M / M / m$, onde $M$ indica distribuição exponencial.

Um ponto importante que surge é o de como processar a carga que entra no sistema, isto é, como distribuir entre os servidores a carga a ser processada.

Para o estudo de possiveis formas de distribuição da carga são necessárias algumas hipóteses. Estas hipóteses são as chamadas hipóteses básicas.

H1. Os jobs são executados individualmente, são logicamente independentes um do outro e podem ser processados por qualquer nó servidor.

H2. As características dos jobs são dadas por uma única distribuição de probabilidade (uma única classe de jobs).

H3. A distribuição dos jobs aos servidores é irrevogável, ou seja, uma vez que um job é designado a um servidor para processamento, ele não pode ser mais tarde redesignado a outro servidor.

H4. O tráfego de mensagem no meio de comunicação tem retardo nulo. Desta forma, o roteamento físico de um job, que pode ser visto como uma mensagem trafegando no meio de comunicação, não vai influir no tempo médio do job no sistema. Esta hipótese é razoável uma vez que, atualmente, a velocidade de transmissão nos meios de comunicação é bastante alta, o que torna o retardo devido à transmissão desprezível.

H5. O 'overhead' devido à comunicação entre processadores é desprezado. Uma vez que cada job é executado integralmente por um servidor, temos que existe apenas a comunicação básica entre processadores. Pela mesma razão citada em H4, vemos que esta hipótese é aceitável e não restritiva. Esta hipótese não seria aceitável, por exemplo, se os jobs fossem divididos em módulos, não necessariamente processados pelo mesmo servidor, pois isto poderia acarretar um aumento muito grande da quantidade de comunicação entre processadores. Este caso foi estudado em [02], [24], [03].

H6. Não existem erros de transmissão e nem de processamento. A detecção e recuperação de erros é uma parte bastante importante do sistema distribuído, mas não será considerada no nosso estudo pois pode ser bastante ampla e complexa, e merece ser estudada 
em separado. Estudos considerando erros de transmissão no sistema foram feitos por [27] e considerando erros de processamento nas tarefas por [02].

H7. O caráter aleatório da demanda e do atendimento cria naturalmente a condição de formação de filas para atendimento, que supomos de capacidade infinita. Assim, estamos assumindo que não há perda de jobs. As filas vão se formar nas origens ou nos servidores, dependendo do modo de atendimento da demanda de serviço, objeto de nossa próxima seção.

Note-se que:

As hipóteses $\mathrm{H} 1$ e H2 garantem que os jobs não são diferenciados sob o ponto de vista do sistema.

As hipóteses $\mathrm{H} 3$ e $\mathrm{H} 6$ foram incluídas a fim de evitar situações de 'deadlock' no sistema.

As hipóteses $\mathrm{H} 4$ e $\mathrm{H} 5$ dizem respeito ao tráfego de mensagens no meio de comunicação.

$\mathrm{E}$ as hipóteses H6 e H7 são importantes para garantir que todos os jobs que entram no sistema são processados.

\subsection{Load Sharing}

O primeiro enfoque para o estudo da influência do projeto no desempenho global de um sistema distribuído será relativo ao fator distribuição da carga entre os servidores.

A distribuição da carga entre os servidores, ou seja, a alocação apropriada dos recursos de processamento do sistema, é também chamada de estratégia de load sharing. Esta estratégia tem como desejáveis as seguintes propriedades:

- Fornecer um desempenho ótimo para o sistema.

A idéia de load sharing é tentar utilizar ao máximo a capacidade de processamento que o sistema oferece, evitando desperdícios de recursos e agilizando o atendimento, isto é, fornecer a máxima vazão com retardos aceitáveis ou mínimo retardo para uma vazão dada;

- Desempenho pouco afetado na presença de falhas parciais do sistema.

No nosso estudo esta propriedade não é relevante, uma vez que temos por hipótese (H6) um sistema sem erros; 
- Tempo de processamento de um job em um dado servidor independente de sua origem, o que é coerente com a hipótese H2.

As estratégias de load sharing podem ser divididas em duas categorias básicas, estática e dinâmica ou adaptativa. Segundo [01], nas estratégias estáticas a distribuição da carga é feita de acordo com regras pré-fixadas, baseadas em informações disponíveis na época do projeto do sistema e que não mudam em função de variação de carga. Isto vem a ser uma desvantagem das estratégias estáticas, pois mudanças das características nominais podem causar sobrecargas em alguns servidores. As estratégias dinâmicas requerem informações sobre o estado do sistema para distribuição da carga. Elas podem ser subdivididas em completas ou incompletas, sendo que essas últimas requerem uma quantidade menor de informação, sendo portanto menos eficientes e podendo inclusive provocar 'deadlock' no sistema. Estas informações são obtidas através de mecanismos que envolvem um 'overhead' significante e a precisão dessas informações dependem do retardo na transmissão. Assim sendo, as estratégias dinâmicas corrigem a desvantagem das estáticas, acima apresentadas, mas são mais difíceis de analisar. Existe ainda uma categoria, denominada semidinâmica, na qual a distribuição da carga é feita baseando-se em algumas informações do passado do sistema. A nossa análise será primordialmente direcionada para estratégias estáticas, pois embora reconheçamos que as estratégias dinâmicas são mais eficientes e mais importantes para a evolução dos sistemas distribuídos, vemos que o estudo e compreensão de estratégias estáticas formam uma base necessária para o estudo das dinâmicas.

As estratégias de load sharing podem ser classificadas quanto ao tipo de nó que toma a iniciativa da alocação dos jobs, isto é, da determinação da ordem de alocação dos jobs para processamento nos servidores ('scheduling') e quanto ao nivel de dependência de informação envolvido na distribuição dos jobs.

Quanto ao tipo de nó que toma a iniciativa da alocação dos jobs, as estratégias estão classificadas em:

- Estratégias com iniciativa do nó origem-onde o nó origem determina qual nó servidor deverá executar um job. Logo, as decisões sobre a alocação são tomadas na chegada e filas tendem a se formar nos servidores.

- Estratégias com iniciativa do nó servidor - onde o nó servidor determina quais jobs, nos diferentes nós origem, cle processará ('server goes looking for work'). Logo, as 
decisões sobre alocação são tomadas nas saídas de jobs do sistema, pois o servidor vai em busca de trabalho quando ele se torna livre e filas tendem a se formar nas origens.

Poder-se-ia conceber estratégias mistas, por exemplo, uma estratégia com iniciativa do nó origem com possibilidade de recusa por parte do servidor. Nós nos concentramos nas duas classes puras acima descritas, inclusive para evitar problemas tipo 'deadlock'.

Confome a descrição acima apresentada, temos que uma estratégia iniciativa do nó origem pode ser descrita por uma função f, que a partir de dadas informações do sistema determina um sevidor, ou seja,

servidor $=\mathrm{f}$ (informações do sistema)

Obviamente, as informações do sistema e a função f podem ser distintas para cada uma das estratégias.

Analogamente, uma estratégia iniciativa do nó servidor pode ser descrita por uma função $f$, tal que

$$
\text { origem }=f(\text { informações do sistema) }
$$

Quanto ao nível de dependência de informação, podemos dizer que uma estratégia (ou um algorítmo) tem nível $i$ de informação quando a função $f$, descrita acima, necessita de $i$ informações sobre o sistema, ou seja, f é uma função de i variáveis.

O nível de dependência de informação indica o quanto um nó origem sabe sobre o status dos servidores, para o caso de estratégias iniciativa do nó origem, ou o quanto um servidor sabe sobre o status das origens, para o caso de estratégias iniciativa do nó servidor. Temos que com um nível maior de informação pode-se obter um desempenho melhor, mas com um custo de comunicação no sistema provavelmente maior.

\subsection{1 - Apresentação das estratégias de load sharing canônicas}

A seguir vamos descrever dez estratégias de load sharing canônicas, sendo cada estratégia descrita por um algoritmo, razão pela qual escreveremos estratégia ou algoritmo indistintamente. Assumiremos que a disciplina nas filas, seja nas origens ou nos servidores, é a FCFS ('First Come First Served'). 
1) Partição das Origens (Source Partition) - $N \geq K$ - O conjunto de nós origens é particionado em grupos e a cada grupo é associado um servidor, de forma que os jobs que entram pelos nós origens de um grupo são por eles enviados, para serem processados, ao nó servidor associado ao grupo.

2) Partição dos Servidores (Server Partition) - $N \leq K$ - O conjunto dos nós servidores é particionado em grupos e cada grupo de servidores processa jobs de uma origem, logo, um job que entrou no sistema é processado por um dos servidores do grupo associado à sua origem.

Este algoritmo pode ser considerado dual do algoritmo partição das origens.

3) Divisấo Aleatória (Random Splitting) - Cada origem distribui os jobs aleatoria e uniformemente aos servidores, isto é, um job é enviado a um dos $\mathrm{K}$ servidores com probabilidade $\frac{1}{K}$

4) Serviço Aleatório (Random Service) - Cada servidor visita uma origem escolhida aleatoria e uniformemente e toma um job dessa origem para processar.

Este algoritmo pode ser considerado dual do algoritmo divisão aleatória.

Pode-se reduzir o overhead de comunicação com a alteração do servidor tomar até B jobs para processamento em cada visita.

5) Divisấo Cíclica (Cyclic Splitting) - Cada origem designa seu i-ésimo job ao servidor $\mathrm{i} \bmod \mathrm{K}$

6) Serviço Cíclico (Cyclic Service) - Cada servidor visita as origens de modo cíclico. Quando o servidor visita a origem i, ele toma até B jobs para processar e a origem i pode ser revisitada até $\mathrm{V}$ vezes ou até que a fila de jobs nesta origem esteja vazia. Então a próxima origem é selecionada.

A sequência de visita e os parâmetros $\mathrm{B}$ e $\mathrm{V}$ podem ser diferentes para cada servidor. Os principais casos são:

$$
\begin{array}{lll}
B=\infty, V=1 & \text { e } & B=1, V=\infty \quad \text { (serviço cíclico exaustivo) } \\
B=1, V=1 & \text { (serviço cíclico limitado) }
\end{array}
$$

$B=1$, limitado $\quad$ (o servidor revisita a origem até que não existam mais jobs na fila ou até que o servidor encontre um job que chegou no sistema após a primeira visita na origem deste ciclo).

Este algoritmo pode ser considerado dual do algoritmo divisão cíclica. 
7) Use o servidor de menor fila (Join The Shortest Queue - JSQ) - Quando um job chega numa origem, ele é enviado ao servidor que tem o menor número de jobs (incluindo o que está sendo processado). No caso de mais de um servidor ter esse menor número de jobs, a escolha de um deles é feita aleatória e uniformemente.

8) Atenda a maior fila (Serve the Longest Queue - SLQ) - Quando um servidor torna-se disponível, ele seleciona um job para ser processado da origem que possui a maior fila. No caso de mais de uma origem possuir a maior fila, a escolha de uma delas é feita aleatória e uniformemente.

9) Prioridade por ordem de chegada (First Come First Served - FCFS) - Quando um servidor torna-se disponível, ele seleciona para ser processado o job que entroutá mais tempo no sistema através de uma das origens.

10) Prioridade para os mais rápidos (Shortest Job First - SJF) - Quando um servidor torna-se disponível, ele seleciona para ser processado o job que requer o menor tempo de processamento.

OBSERVAÇÂA: Para os algoritmos de 7) a 10) usaremos as iniciais em inglês por serem o padrão da literatura.

Estas dez estratégias vão ser classificadas quanto à iniciativa de alocação dos jobs e quanto ao nível de informação, conforme a Tabela I.

Quanto ao nível de informação, em [29] foi dito que as estratégias com nível 1 são estáticas, as de nível 3 são semidinâmicas, as de nível 5 são dinâmicas e os demais níveis podem ser considerados níveis intermediários. 


\section{TABELA I}

\begin{tabular}{|c|c|c|c|}
\hline estratégia & descrição da estratégia & \multicolumn{2}{|c|}{$\begin{array}{l}\text { iniciativa da nível de } \\
\text { schedulagem inf. }\end{array}$} \\
\hline Partição das Origens & servidor $=\mathrm{f}($ origem $)$ & origem & 1 (estática) \\
\hline Partição dos Servidores & origem $=\mathrm{f}($ servidor $)$ & servidor & 1 (estática) \\
\hline Divisão Aleatória & servidor $=\mathrm{f}($ origem,$\omega)$ & origem & 2 \\
\hline Serviço Aleatório & origem $=\mathrm{f}($ servidor,$\omega)$ & servidor & 2 \\
\hline Divisão Cíclica & servidor $=\mathrm{f}($ origem,$\omega$, sequencialização $)$ & origem & 3 (semidin.) \\
\hline Serviço Cíclico & origem $=\mathrm{f}($ servidor,$\omega$, sequencialização $)$ & servidor & 3 (semidin.) \\
\hline JSQ & $\begin{array}{c}\text { servidor }=\mathrm{f}(\text { origem, } \omega, \text { sequencialização, } \\
\text { comprimento das filas dos servidores })\end{array}$ & origem & 4 \\
\hline SLQ & $\begin{array}{c}\text { origem }=\mathrm{f} \text { (servidor, } \omega \text {, sequencialização, } \\
\text { comprimento das filas das origens })\end{array}$ & servidor & 4 \\
\hline FCFS & $\begin{array}{l}\text { origem }=\mathrm{f} \text { (servidor, } \omega \text {, sequencialização, } \\
\text { época de chegada dos jobs nas origens) }\end{array}$ & servidor & 4 \\
\hline SJF & $\begin{array}{l}\text { origem }=\mathrm{f} \text { (servidor, } \omega \text {, sequencialização, } \\
\text { época de chegada dos jobs nas origens, } \\
\text { tempos de execução dos jobs) }\end{array}$ & servidor & 5 (dinâmica) \\
\hline
\end{tabular}

Existem exemplos de outros algoritimos estáticos, que não foram considerados na Tabela I e que foram estudados e modelados como problemas de programação matemática ou de fluxo em redes e cujas soluções foram obtidas através de otimização em relação à alguma medida de desempenho do sistema, considerando a carga total do sistema fixa e conhecida (isto porque, conforme já citado, algoritmos estáticos não respondem à variação de carga). Três deles foram apresentados em [24], [03] e [02] e consideram basicamente o problema de alocar módulos de programas que se comunicam a processadores, de modo a obter o menor custo de comunicação possível (considera-se que um programa é dividido em um ou mais módulos).

A resolução desse problema para dois processadores é feita através da construção de 
um grafo de comunicação onde os nós são os módulos e os processadores e as arestas existem entre módulos comunicantes, com peso associado igual ao custo de comunicaçâo entre eles e entre cada nó processador e todos os módulos, com peso associado igual ao custo de rodar o módulo no outro processador (por exemplo, a aresta que liga o processador P1 ao módulo $\mathrm{X}$ tem peso igual ao custo de rodar X no processador P2). A solução do problema é obtida executando o algoritmo de mínimo corte no grafo de comunicação. Nota-se que a medida de desempenho é dada somente pelo custo de comunicação, não sendo considerada a vazão ou o tempo médio no sistema. Em cada um dos autores foram consideradas variações desse problema básico.

$\mathrm{Na}$ verdade, existem na literatura, vários algoritmos de load sharing, sendo que em geral, eles podem ser vistos como variações ou misturas dos algoritmos canônicos. Assim, os algorítmos canônicos formam uma parte bastante representativa do conjunto geral das estratégias.

Nós nos restringiremos a apresentar uma parte dos estudos e observações sobre os algoritmos canônicos e seu desempenho feitos em [29], sendo que para maiores detalhes e informações recomendamos sua consulta.

Após a apresentação desse estudos, conforme já mencionado, nos concentraremos nas estratégias canônicas com nível 1 de informação, mais precisamente, nas estratégias estáticas partição das origens e partição dos servidores. A análise dessas estratégias vem a ser o ponto principal dos capítulos 3 e 4 .

\subsection{2 - Análise das estratégias canônicas}

Além das hipóteses básicas já apresentadas, serão assumidas as seguintes hipóteses:

- A entrada de jobs no sistema, via uma origem i, forma um processo Poisson com taxa média $\lambda_{i}$. Na verdade, este processo Poisson descreve uma coleção de chegadas tais que os tempos entre-chegadas são independentes e distribuídos exponencialmente com média $\frac{1}{\lambda_{i}}$.

- A distribuição de probabilidade do tempo de atendimento de um servidor do sistema é a exponencial e é a mesma para todos os servidores com média $\bar{\mu}^{-1}$. A hipótese da igualdade da distribuição foi considerada no trabalho apresentado em [29], e portanto 
será utilizada neste capítulo.

Vale lembrar que, segundo as hipóteses básicas, o retardo devido á comunicação não tem efeito sensível na análise.

A análise de um algoritmo canônico pode ser auxiliada por uma modelagem que descreva o problema. Alguns dos algoritmos canônicos podem ser modelados por sistemas de filas, sendo que alguns modelos possuem solução exata e outros foram resolvidos por simulação ou por aproximação em [29]. Neste ponto serão apresentados esses algorítmos com suas respectivas modelagens e exemplos representados esquematicamente.

O algoritmo Partição das Origens pode ser modelado por um sistema composto por $\mathrm{K}$ filas $M / M / 1$.

Exemplo para $N=5$ e $K=2$

origens
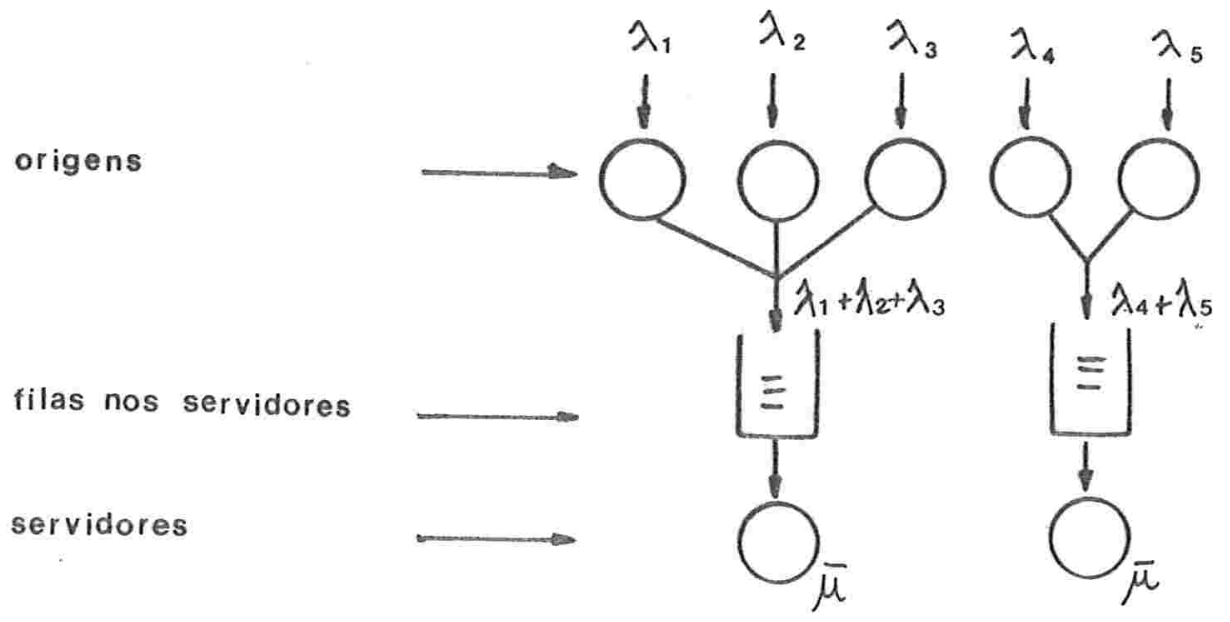

O algoritmo Partição dos Servidores pode ser modelado por um sistema composto por $\mathrm{N}$ filas $M / M / c_{j}$, onde $c_{j}$ é o número de servidores associados à origem $\mathrm{j}$, para $j=$ $1,2, \cdots, N$ e $\quad c_{1}+c_{2}+\cdots+c_{N}=K$. 
Exemplo para $N=2$ e $K=3$

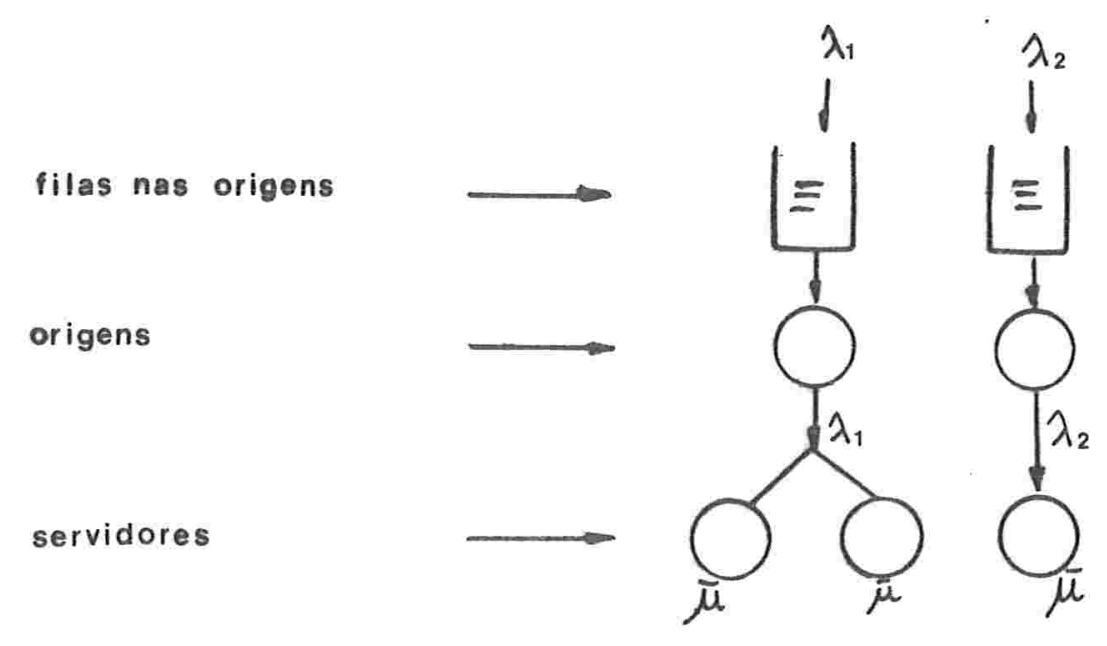

$\mathrm{O}$ algoritmo Divisão Aleatória pode ser modelado por um sistema composto por $\mathrm{K}$ filas $M / M / 1$.

Exemplo para $N=2$ e $K=3$

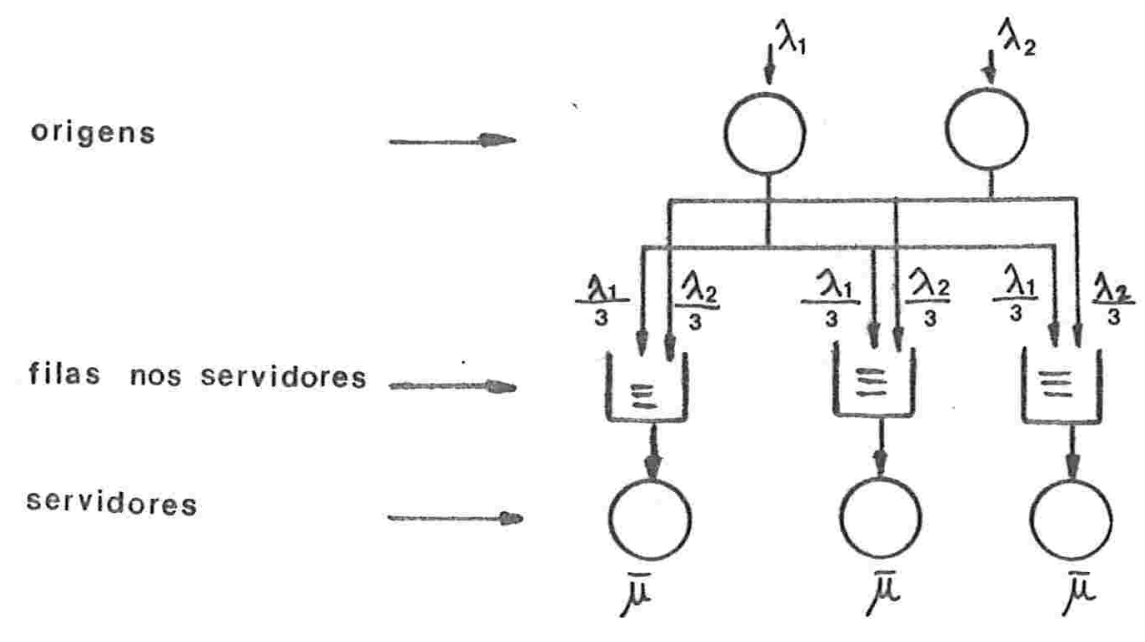

O algoritmo Divisão Cíclica pode seṛ modelado por um sistema composto por $\mathrm{K}$ filas $\sum_{i=1}^{N} E_{K}\left(\lambda_{i}\right) / M / 1$, onde $E_{K}\left(\lambda_{i}\right)$ é uma distribuição Erlang com $K$ estágios e taxa média $\lambda_{i}$, que é na verdade uma coleção estruturada de distribuições exponenciais com taxa média $\lambda_{i}$. Segundo a distribuição $E_{K}\left(\lambda_{i}\right)$, um job entra no sistema no estágio 1 de uma estação de entrada e permanece neste estágio por um tempo aleatório, dado pela distribuição exponencial com taxa média $\lambda_{i}$, passando então para o estágio 2. E assim sucessivamente até chegar ao estágio $\mathrm{K}$, onde ele permanece por um tempo aleatório dado pela distribuição exponencial com taxa média $\lambda_{i}$ e então sai da estação e entra na fila. Um 
novo job não entra na estação até que o anterior saia, ou seja, enquanto a estação estiver 'ocupada'. Desta forma, simula-se o fato de que uma vez que um job seja designado a um servidor, somente o K-ésimo job a partir dele será designado ao mesmo servidor, pois os jobs anteriores ao K-ésimo encontrarão a estação 'ocupada', e assim por diante. Logo, a distribuição Erlang é utilizada para simular o ciclo dos servidores. Note-se que o s!mbolo $\Sigma$ denota superposição de processos.

Exemplo para $N=2$ e $K=3$
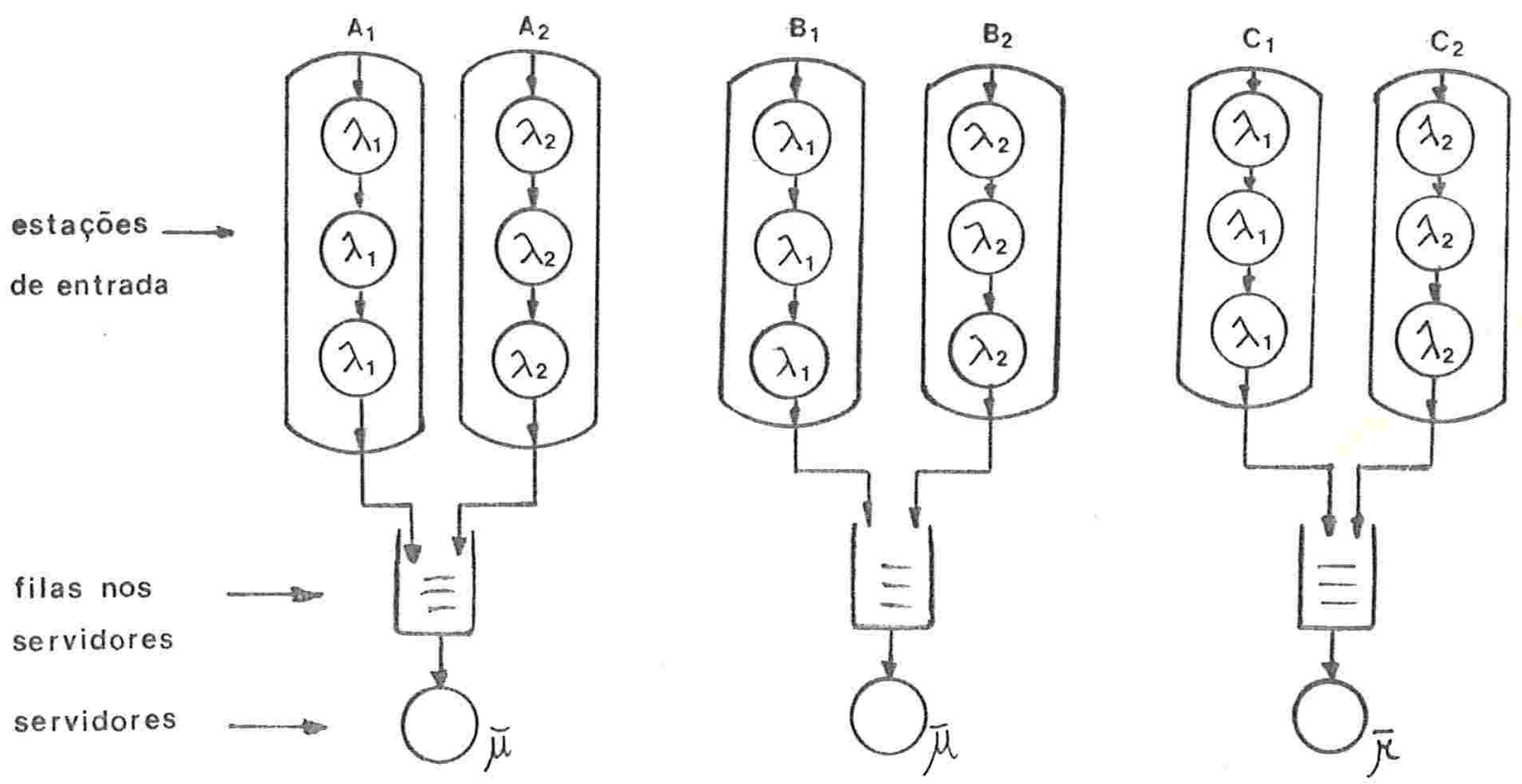

Um job que entra no sistema através da origem 1 é enviado à primeira das estações de entrada, entre A1, B1 e C1 nesta ordem, que não esteja 'ocupada'. Analogamente para a origem 2 e as estações A2, B2 e C2.

Para maiores detalhes sobre a distribuịção Erlang ver [16].

O algoritmo $F C F S$ pode ser modelado por um sistema composto por $N$ filas $M / M / K$. 
Exemplo para $N=2$ e $K=4$

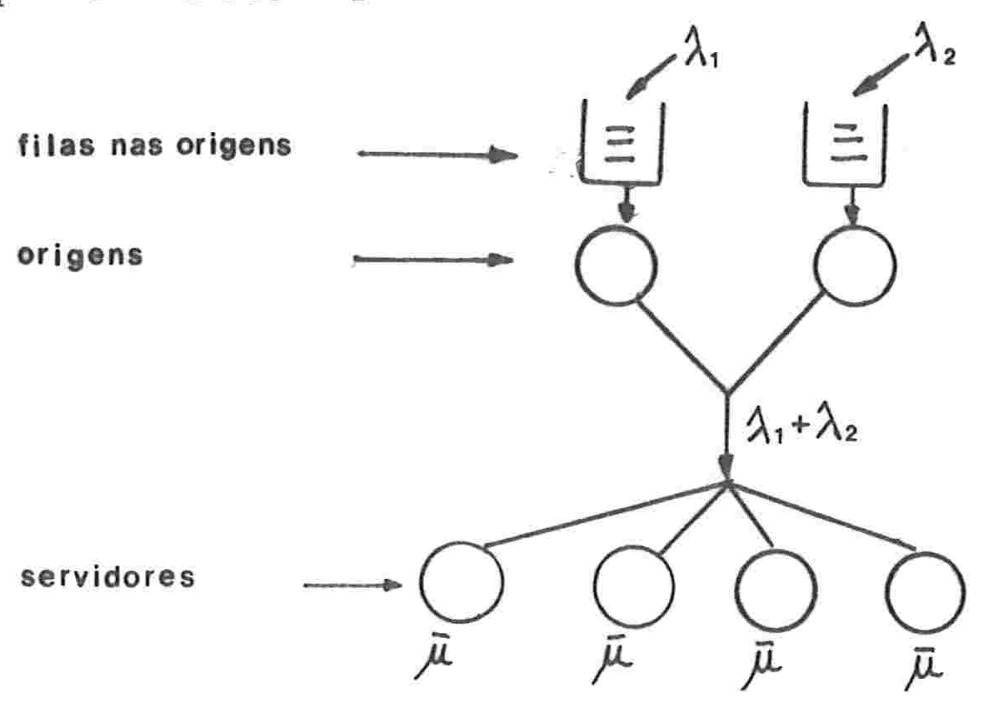

Os demais algoritmos (Serviço Aleatório, Serviço Cíclico, JSQ, SLQ, SJF) são analisados em [29], em geral, através de simulações ou aproximações.

A medida de desempenho utilizada para análise dos algoritmos canônicos é o tempo médio no sistema ( $\mathrm{T})$, que é dado por

$$
T=\sum_{i} \frac{\lambda_{i}}{\lambda} T_{i}
$$

onde $T_{i}$ é o tempo médio no sistema para jobs que entraram pela origem i.

Existe na literatura, vide [16] e [30], uma expressão exata para tempo médio num sistema que consiste de uma fila $M / M / m$, logo a análise dos algoritmos Partição das Origens, Partição dos Servidores, Divisão Aleatória e FCFS é exata. Para uma taxa de entrada $\bar{\lambda}$, a expressão é

$$
\begin{aligned}
T_{m} & =\bar{\mu}^{-1}+\frac{(m \rho)^{m} P_{0}}{m ! m \bar{\mu}(1-\rho)^{2}} \\
\text { onde } \quad \rho & =\frac{\bar{\lambda}}{m \bar{\mu}} \mathrm{e} \\
P_{0} & =\left[\frac{(m \rho)^{m}}{m !(1-\rho)}+\sum_{n=0}^{m-1} \frac{(m \rho)^{n}}{n !}\right]^{-1}
\end{aligned}
$$

Para o algoritmo Divisão Cíclica não existe expressão exata para o tempo médio no 
sistema e é feita uma análise aproximada.

Conforme já descrito, o algorítmo Divisão Cíclica pode ser modelado por filas do tipo $\sum_{i=1}^{N} E_{K}\left(\lambda_{i}\right) / M / 1$ e portanto o tempo médio no sistema é dado pelo tempo médio numa dessas filas. Segundo [31], valem as seguintes considerações:

- O coeficiente de variação (c) da distribuição do processo superposto (processo este que define a entrada na fila) pode ser aproximado por

$$
c^{2}=\omega c_{a}^{2}+(1-\omega) c_{m}^{2}
$$

onde

$c_{a}$ é o coeficiente de variação calculado pelo método assintótico e é dado por

$$
c_{a}^{2}=\sum_{i=1}^{N} \frac{\lambda_{i}}{\sum_{j=1}^{N} \lambda_{j}} c_{i}^{2}
$$

$c_{i}$ é o coeficiente de variação do processo i definido pela distribuição $E_{K}\left(\lambda_{i}\right)$, $i=1,2, \cdots, N$.

$c_{m}$ é o coeficiente de variação da distribuição exponencial

$$
\begin{aligned}
& \omega=\left[1+2.1(1-\rho)^{1,8} \nu\right]^{-1} \\
& \nu=\left[\sum_{i=1}^{N}\left(\frac{\lambda_{i}}{\sum_{j=1}^{N} \lambda_{j}}\right)^{2}\right]^{-1}
\end{aligned}
$$

Vale lembrar que o coeficiente de variação de uma distribuição é dado pelo quociente de seu desvio padrão, que é a raiz quadrada da variância, pela sua média.

Assim temos $c_{m}=1$ e $c_{i}=\frac{\sqrt{K}}{K}$, e portanto $c_{a}^{2}=\frac{1}{K}$.

Logo,

$$
c^{2}=\omega c_{a}^{2}+(1-\omega)=1+\omega\left(\frac{1}{K}-1\right) \leq 1
$$

- O tempo médio de espera na fila $\sum_{i=1}^{N} E_{K}\left(\lambda_{i}\right) / M / 1$ pode ser aproximado por

$$
E W=\bar{\mu}^{-1} \rho\left(c^{2}+1\right) g / 2(1-\rho)
$$


onde

$g=\exp \left(\frac{-2(1-\rho)}{3 \rho} \frac{\left(1-c^{2}\right)^{2}}{c^{2}+1}\right)$

$\rho$ - fator de utilização do sistema que consiste desta fila.

Então,o tempo médio no sistema pode ser aproximado por

$$
\hat{T}_{D C}=\bar{\mu}^{-1}+E W
$$

Naturalmente, existe erro na aproximação assim obtida, mas segundo [31], tanto a aproximação para o coeficiente de variação c quanto a aproximação para tempo médio de espera na fila, se comportam relativamente bem.

Para o algorítmo JSQ, temos que um job que entra no sistema pode ser processado por qualquer dos $\mathrm{K}$ servidores, sendo escolhido o que tem menor fila. O tempo médio no sistema, é dado em [29], baseado em aproximações feitas em [21] e [08].

$$
\hat{T}_{J S Q}=\bar{\mu}^{-1}+\frac{\lambda^{K}\left[K+\left(1-\frac{1}{K}\right) \lambda\right]}{d(\lambda)}
$$

onde $d(\lambda)=(K-\lambda)\left[K !\left(1-\frac{\lambda}{K}\right) \sum_{\ell=0}^{K-1} \frac{\lambda^{\ell}}{\ell !}+\lambda^{K}\right]$

Também foi observado por Y.T.Wang [29], que o erro dessa aproximação em relação a resultados obtidos por simulação cresce com $\rho$ e também cresce com K. Para valores pequenos de $K$, o erro na aproximação pode ser considerado aceitável.

\subsubsection{1 - Análise com carga homogênea}

Neste item mostramos a comparação gráfica de alguns dos algoritmos canônicos, quanto a tempo médio no sistema como função do fator de utilização global do sistema, considerando a carga homogênea, isto é, a mesma carga em todas as origens.

Para cada um dos gráficos da FIG. 2.1 indicamos a expressão utilizada para tempo médio no sistema.

O gráfico do algoritmo Partição dos Servidores (PS) é o gráfico da função T, definida a seguir. 


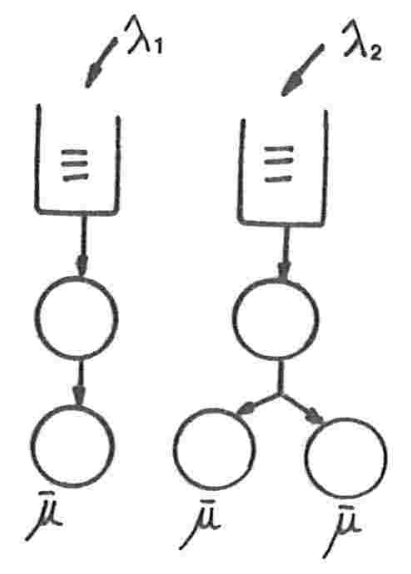

$$
\begin{aligned}
T & =\frac{\lambda_{1}}{\lambda} T_{1}+\frac{\lambda_{2}}{\lambda} T_{2}=\frac{1}{2}\left(T_{1}+T_{2}\right) \\
T_{1} & =\frac{1}{\bar{\mu}\left(1-\rho_{1}\right)}=\frac{1}{1-\rho_{1}} \quad \text { onde } \quad \rho_{1}=\frac{\lambda_{1}}{\bar{\mu}}=\frac{\lambda}{2} \\
T_{2} & =\frac{1}{\bar{\mu}\left(1-\rho_{2}^{2}\right)}=\frac{1}{1-\rho_{2}^{2}} \quad \text { onde } \quad \rho_{2}=\frac{\lambda_{2}}{2 \bar{\mu}}=\frac{\lambda}{4}
\end{aligned}
$$

Obviamente devemos ter $\rho_{1}<1$ e $\rho_{2}<1$ para que não ocorra saturação de uma das filas, o que implica em termos a carga total $\lambda<2$.

O gráfico do algorítmo Divisão Aleatória (DA) é dado pela função T abaixo

$$
T=\frac{1}{\bar{\mu}} \frac{1}{1-\tilde{\rho}} \quad \text { onde } \quad \tilde{\rho}=\frac{\tilde{\lambda}}{\bar{\mu}}=\frac{\lambda}{3}
$$

Analogamente, devemos ter $\tilde{\rho}<1$, ou equivalentemente, $\lambda<3$.

O gráfico do algoritmo Divisão Cíclica (DC) é dado pela função T abaixo

$$
\begin{aligned}
T & =1+\rho\left(c^{2}+1\right) g / 2(1-\rho) \\
\operatorname{com} g & =\exp \left(\frac{-2(1-\rho)}{3 \rho} \frac{\left(1-c^{2}\right)^{2}}{c^{2}+1}\right) \\
c^{2} & =1-\frac{2}{3}\left[\frac{1}{1+4.2(1-\rho)^{1.8}}\right] \\
\rho & =\frac{\lambda}{3}
\end{aligned}
$$

O gráfico do algoritmo JSQ é o gráfico da função que é uma aproximação para o tempo médio no sistema, já definida anteriormente, que aplicada aos valores de $\mathrm{N}, \mathrm{K}, \bar{\mu}$ considerados é 


$$
T=1+\frac{\lambda^{3}\left(3-\frac{2}{3} \lambda\right)}{d(\lambda)}
$$

$\operatorname{com} d(\lambda)=(3-\lambda)\left[(6-2 \lambda)\left(1+\lambda+\lambda^{2}\right)+\lambda^{3}\right]$.

O gráfico do algoritmo FCFS é dado pela função T abaixo

$$
T=\frac{1}{\bar{\mu}}\left[1+\frac{3 \rho^{3}}{\left(2+4 \rho+3 \rho^{2}\right)(1-\rho)}\right] \quad \text { onde } \quad \rho=\frac{\lambda}{3 \bar{\mu}}=\frac{\lambda}{3}
$$

Analogamente, devemos ter $\rho<1$, ou equivalentemente, $\lambda<3$. Vale lembrar que este também é o gráfico dos algoritmos Serviço Aleatório, Serviço Cíclico e SLQ, conforme observação anterior.

postegar $(4123)$

As seguintes interpretações, quanto ao desempenho dos algoritmos canônicos são apresentadas em [29]:

- O uso do número de jobs numa fila como indicação de carga é inadequado, uma vez que esse dado não fornece informação sobre os jobs na fila, podendo ocorrer a situação de jobs esperando por um servidor enquanto um outro servidor está livre. Isto explica o fato do algoritmo JSQ ter um desempenho pior que o FCFS.

- Nos algoritmos Divisão Aleatória e Divisão Cíclica pode ocorrer com maior frequência a existência de servidores livres com jobs esperando nas filas, isto porque esses algoritmos não conhecem os estados dos servidores, e portanto esses algoritmos têm seu desempenho deteriorado, sendo que o algoritmo Divisão Cíclica tem um desempenho um pouco melhor que o de Divisão Aleatória, devido à regularidade introduzida pelo ciclo dos servidores, que faz com que todos os servidores recebam cargas semelhantes.

- Entre os algoritmos canônicos, é esperado que o algoritmo SJF tenha o melhor desempenho, uma vez que é o único algoritmo que conhece os tempos de serviço requeridos pelos jobs (tem um nível de informação alto). Uma motivação forte para esta observação é que uma fila M/G/1 (G-distribuição geral), com disciplina de atendimento $\mathrm{SJF}$, onde um job que tenha um tempo de processamento conhecido de $\mathrm{x}$ segundos, junta-se à fila atrás de todos os jobs cujos tempos de processamento são menores que $\mathrm{x}$, fornece o menor tempo médio de espera quando comparada com outras disciplinas de atendimento, tais como FCFS, LCFS etc. (vide [16]). 
- Os algoritmos iniciativa do servidor têm desempenho melhor que os algoritmos iniciativa da origem com mesmo nível de informação. Isto porque nos algoritmos iniciativa do servidor não ocorre de existirem servidores livres e jobs esperando para serem atendidos, pois assim que um servidor fica livre vai em busca de novo trabalho. Este fato pode ser observado, por exemplo, para os algoritmos canônicos nível 2 (Divisâo Aleatória e Serviço Aleatório).

- Os algoritmos Serviço Aleatório, Serviço Cíclico $(B=1)$, SLQ e FCFS fornecem mesmo tempo médio no sistema, portanto eles não são diferenciados por essa medida de desempenho escolhida. Isto vem do fato de que um job que entra no sistema pode ser processado por qualquer dos $\mathrm{K}$ servidores, pois cada servidor atende a todas origens, sendo os algoritmos diferenciados apenas por uma mudança da ordem de processamento dos jobs, lembrando que no algoritmo Serviço Aleatório um servidor atende às origens aleatoriamente, no algoritmo Serviço Cíclico um servidor atende às origens de modo ć́clico, no algoritmo SLQ um servidor atende à origem de maior fila e no algoritmo FCFS um servidor atende à origem que possui o job que chegou ${ }^{H}$ á mais tempo no sistema. Assim, pelo resultado de Little (vide [16]), vem que todos esses algoritmos fornecem mesmo tempo médio no sistema. Este resultado também pode ser entendido ao observarmos que uma fila $\mathrm{M} / \mathrm{M} / \mathrm{K}$ pode ser vista como um processo vida e morte.

Estes algoritmos podem ser comparados sob outros pontos de vista ou até mesmo por várias medidas de desempenho a fim de serem diferenciados. 
FIG. 2.1

$$
N=2 \quad K=3 \quad \pi^{-1}=1 \quad \lambda_{1} / \lambda_{2}=1
$$

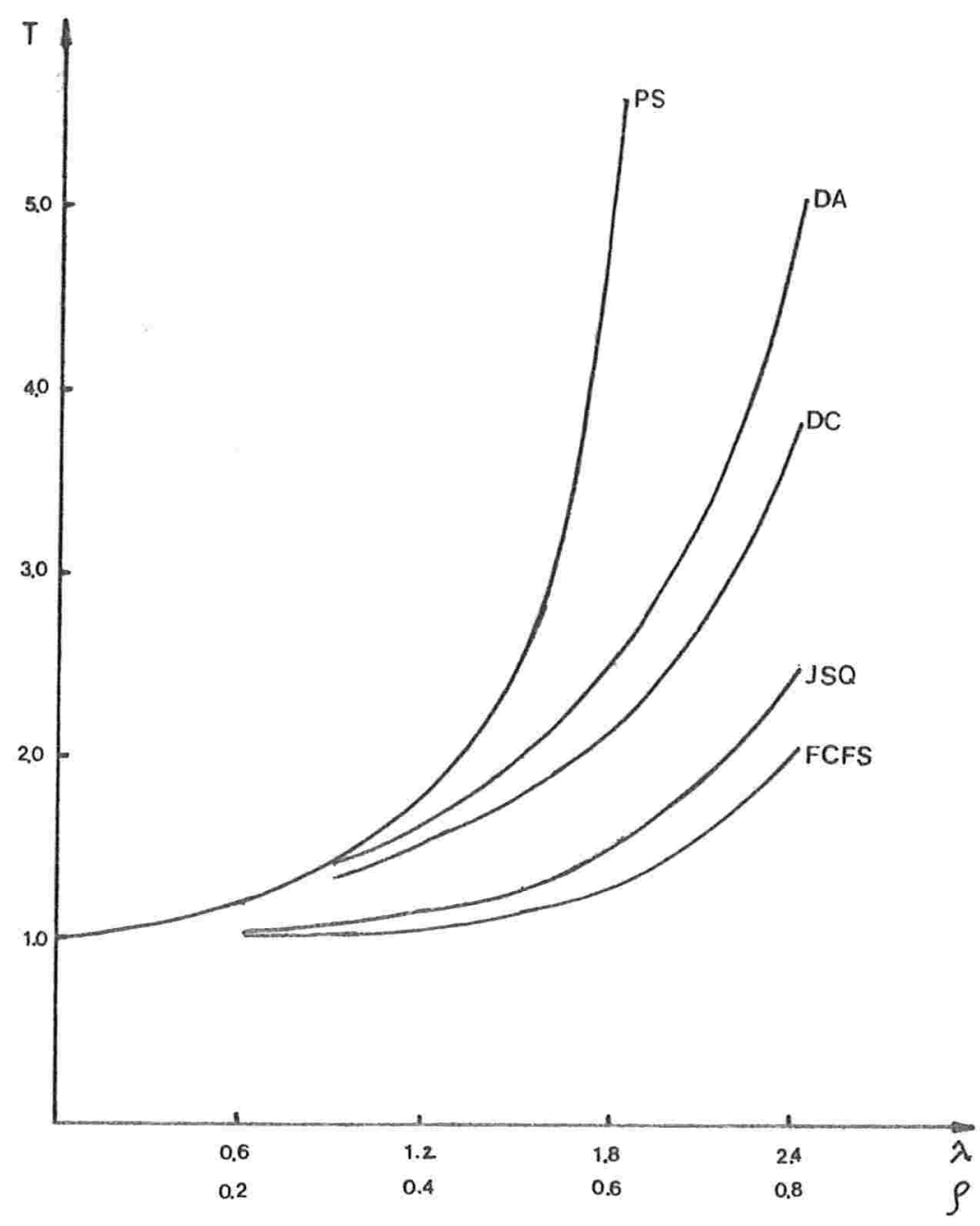




\subsubsection{Análise com carga não homogênea}

A té aqui foi considerado o desempenho dos algoritmos canônicos considerando-se carga homogênea. Vamos agora considerar o desempenho desses algoritmos com carga não homogênea, de forma a verificar o efeito da variação da divisão da carga entre as origens no desempenho global desses algoritmos.

A análise desse efeito foi feita em [29], em geral, com a utilização de simulações. A seguir apresentaremos as principais conclusões por ele obtidas, consistentes com a modelagem utilizada (Markoviana).

\section{1) Efeito no algoritmo Partição dos Servidores}

Este algoritmo é bastante afetado pela variação da distribuição da carga entre as origens. Para sentir este efeito, utilizamos o caso $N=2, K=3, \mu^{-1}=1$ e consideramos várias possíveis distribuições da carga, seguindo o seguinte esquema.

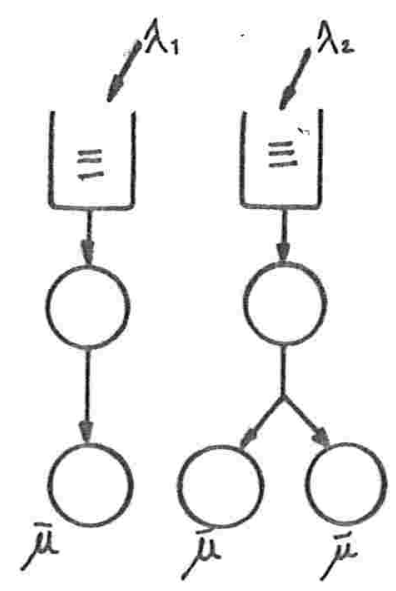

$\operatorname{com} \quad \lambda_{2}=p \lambda_{1} \quad$, ou seja, $\quad \lambda_{1}=\frac{1}{p+1} \lambda \quad$ e $\quad \lambda_{2}=\frac{p}{p+1} \lambda$

Temos que o tempo médio no sistema é dado por

$$
\begin{aligned}
T & =\frac{\lambda_{1}}{\lambda} T_{1}+\frac{\lambda_{2}}{\lambda} T_{2}=\frac{1}{p+1}\left(T_{1}+p T_{2}\right) \\
T_{1} & =\frac{1}{\bar{\mu}\left(1-\rho_{1}\right)}=\frac{1}{1-\rho_{1}} \quad \text { onde } \quad \rho_{1}=\frac{\lambda}{(p+1) \bar{\mu}}=\frac{\lambda}{p+1} \\
T_{2} & =\frac{1}{\bar{\mu}\left(1-\rho_{2}^{2}\right)}=\frac{1}{1-\rho_{2}^{2}} \quad \text { onde } \quad \rho_{2}=\frac{p \lambda}{(p+1) 2 \bar{\mu}}=\frac{p \lambda}{2(p+1)}
\end{aligned}
$$


Devemos ter as filas insaturadas, ou seja, $\rho_{1}<1$ e $\rho_{2}<1$.

$\mathrm{O}$ caso $p=0$ corresponde a colocar toda a carga na origem 1 . O caso $p=\infty$ corresponde a colocar toda a carga na origem 2 , sendo que para esse caso as expressões acima não podem ser consideradas e a expressão para $\mathrm{T}$ é dada por

$$
T=\frac{1}{\bar{\mu}\left(1-\left(\frac{\lambda}{2 \bar{\mu}}\right)^{2}\right)}=\frac{1}{1-\left(\frac{\lambda}{2}\right)^{2}}
$$

Na FIG. 2.2 observamos que os melhores tempos são conseguidos com uma carga maior na origem que está associada ao maior número de servidores e ainda que o algoritmo FCFS tem desempenho melhor que o algoritmo Partição dos Servidores, independentemente da distribuição da carga entre as origens.

Podemos então perceber a grande influência da distribuição da carga entre os servidores no desempenho do algoritmo Partição dos Servidores. Este fato nos motivou a estudar com mais detalhe esse algoritmo e os vários fatores que influem no seu desempenho. Este estudo será objeto dos próximos capítulos. 


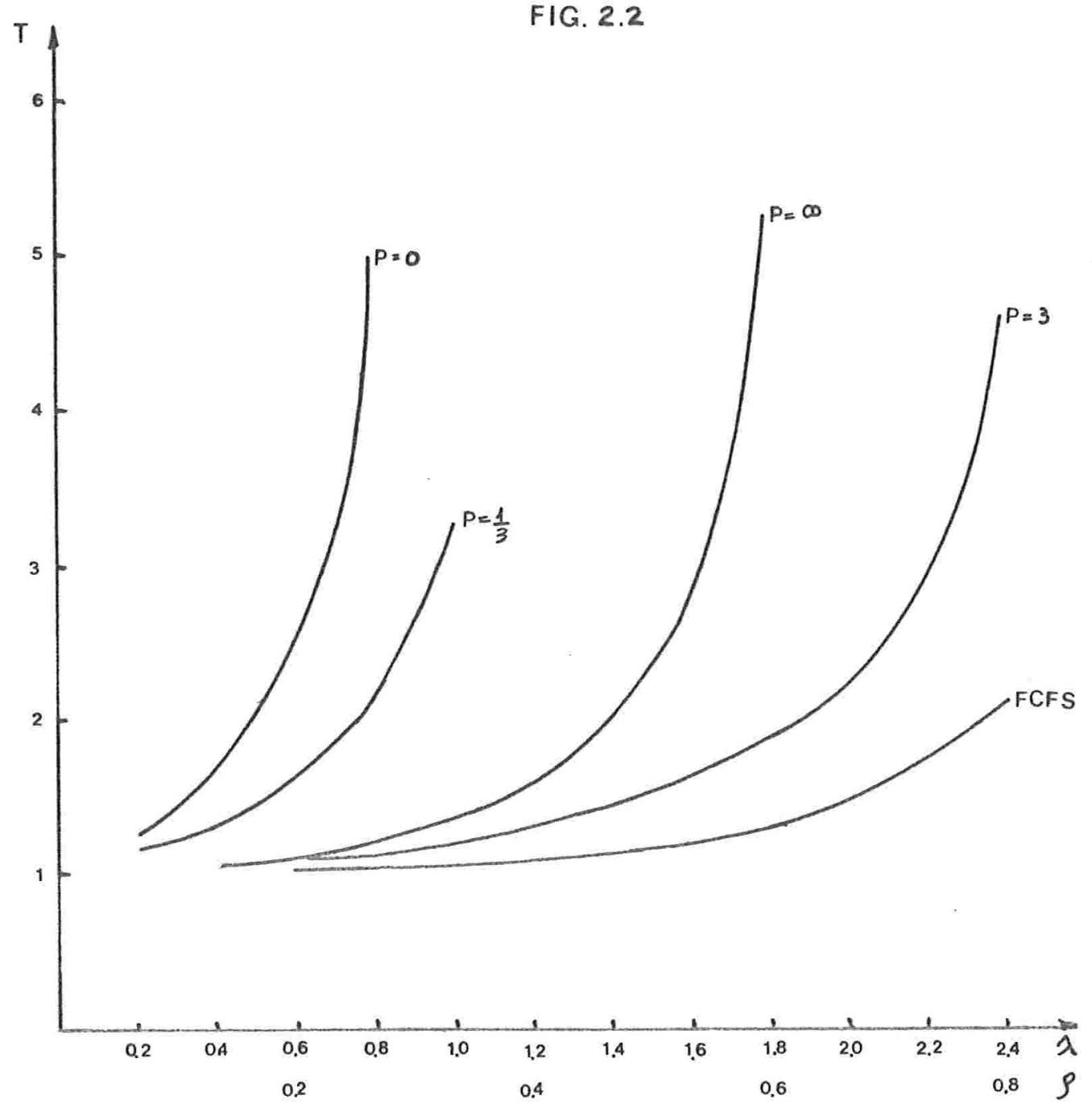


2) O algoritmo $J S Q$ não é afetado pela variação da carga entre as origens, isto pois a escolha do servidor que processará os jobs independe da origem do job.

3) O algoritmo $S L Q(K \geq 2)$ degrada com não homogeneidade de carga, devido ao fato de que as origens menos carregadas têm baixa prioridade em ganhar serviço. Este fato pode ser percebido facilmente quando temos uma origem com carga média próxima de zero, uma vez que um job que chega nesta origem só vai ser atendido por um dos servidores quando todas as demais filas nas origens tiverem menos jobs que a origem em questão.

4) O algoritmo Serviço Cíclico $(B=1, V=\infty)$ também degrada com não homogeneidade de carga, isto pois um job que chega numa origem de baixa carga tem que esperar até que esta origem seja selecionada no ciclo, e para que isto ocorra toas as origens anteriores a ela no ciclo devem ter tido suas filas esvaziadas.

5) O algoritmo Divisâo Aleatória não é afetado pela variação da distribuição da carga entre as origens, isto pois a origem do job não é relevante para o algoritmo. Já o algoritmo Divisão Cíclica tem seu desempenho ligeiramente afetado.

6) Os algoritmos Serviço Aleatório, FCFS, SJF também não são afetados pela variação da distribuição da carga.

\subsubsection{Comentários adicionais}

- Efeito do 'overhead' devido à comunicação entre processadores.

A análise de desempenho dos algoritmos se torna bastante difícil quando se considera que o efeito do 'overhead' devido à comunicação entre processadores é não desprezível. Isto porque este efeito num algoritmo depende de sua implementação e é bastante subjetivo.

Vale lembrar que este efeito aumenta à medida que aumenta o nível de informação de um algoritmo. Assim, podemos verificar que, entre os algoritmos iniciativa da origem, o algoritmo JSQ tem o melhor desempenho, quando se despreza o efeito do 'overhead', mas por outro lado, é o que envolve o maior 'overhead'. 
- Análise de desempenho de algoritmos com relação a uma nova medida definida.

Em [29] foi introduzida uma medida para avaliar o desempenho dos algoritmos canônicos, definida por

$$
Q_{A}(\rho)=\frac{\text { tempo médio no sistema sobre FCFS }}{\sup _{\frac{\sum_{i=1}^{N \bar{\mu}} \lambda_{i}}{N}=\rho} \max _{i}\{\text { tempo médio no sistema sobre algoritmo A, na origem i }\}}
$$

Essa medida fornece um fator que mostra o quão próximo os algoritmos estão do algoritmo FCFS. Esta definição vem do fato que o algoritmo FCFS tem um comportamento que emula o conceito intuitivo usual de processamento distribuido, pois possui as seguintes características:

- Não permite que qualquer servidor fique livre com jobs esperando para serem processados;

- Não discrimina jobs em função das suas origens.

Assim, essa medida tem o intuito de considerar o comportamento global do sistema, em relação à distribuição da carga, detectando as características acima apresentadas, que constituem ineficiências do sistema.

Note-se que para calcular o Q para um algoritmo é necessário encontrar a combinação de cargas nas origens que causa o pior desempenho, o que não necessariamente é um trabalho simples.

O autor sugeriu a utilização dessa medida para analisar os algoritmos Serviço Cíclico $(B=1)$, SLQ, Serviço Aleatório e FCFS, que têm o mesmo comportamento com cargas homogêneas. Também mostrou que o Q para o algoritmo Serviço Cíclico $(B=1, V=\infty)$ é igual ao Q para o algoritmo SLQ. Para esses dois algoritmos, o Q é determinado quando a carga numa das origens tende a zero $\left(\lambda_{i} \rightarrow 0\right.$ para algum i). O autor ainda observou que a maioria dos algoritmos degradam (Q diminui) à medida que $\rho \rightarrow 1$, sendo que os algoritmos Serviço Cíclico possuem Q's razoáveis, exceto para $\rho \rightarrow 1$. A melhor versão desses algoritmos é a $B=1$, limitado. Nada foi dito a respeito do $\mathrm{Q}$ do algoritmo Serviço Aleatório. 


\section{Críticas:}

A expressão $\max _{i}\{$ tempo médio no sistema sobre algoritmo $A$, na origem i fornece o pior tempo médio no sistema para um determinado job, pois considera o tempo médio no sistema para esse job quando ele entra por cada uma das origens. Logo, esta expressão define o usuário pior atendido pelo sistema, se considerarmos cada origem como sendo um terminal e um usuário requisitando o processamento do job nesse terminal. Isto pode ser considerado compativel com uma proposta de medida de desempenho.

O supremo colocado a nível mais externo do denominador considera todas as possíveis distribuições de carga que fornecem um dado fator de utilização do sistema e toma a pior delas, no sentido que ela fornece o pior tempo médio no sistema para um job. Isto pode ter pouco sentido prático, pois pressupõe que os terminais (origens) estão muito mal distribuídos e que portanto o sistema está mal projetado. Seria mais razoável supormos um projeto inteligente com terminais alocados da melhor forma possível, tomando inf ao invés de sup.

Um outro ponto a ser discutido a respeito dessa medida é a respeito da dificuldade em encontrar a pior distribuição de carga que implica, na verdade, na resolução de um problema de otimização.

- Considerações sobre o algoritmo Serviço Cíclico.

Algumas considerações interessantes serão feitas a respeito do algoritmo Serviço Cíclico, baseados no trabalho de [18].

Primeiramente lembramos que o algoritmo Serviço Cíclico $(B=1)$ tem o mesmo desempenho que o algoritmo FCFS, com cargas homogêneas, e tem seu desempenho degradado com não homogeneidade de cargas.

Se, ao escolhermos um algoritmo de 'load sharing', considerarmos custo de comunicação desprezível é melhor tomarmos um algoritmo que use maior quantidade de informação, de forma a obter menor tempo médio no sistema. Se considerarmos que o custo de comunicação não pode ser desprezado em relação ao tempo de execução, o algoritmo Serviço Cíclico pode ser interessante.

No algorítmo Serviço Cíclico, cada servidor visita as origens de modo cíclico. O ciclo 
de um servidor é definido por uma rota a ser seguida para visita às origens, ou seja, é uma ordem para percorrer as origens e é dada por uma permutação dos inteiros $1,2, \ldots, N$. Esta rota pode ser diferente para cada servidor.

Em [18] foi observado que o desempenho do algoritmo serviço cíclico é afetado pelas rotas ć́clicas dos servidores. Se todos os servidores seguem a mesma rota, existe uma tendência deles se aglutinarem deteriorando o tempo médio no sistema dos jobs. Logo, é desejável que os servidores tenham diferentes rotas durante seus ciclos de modo a atenderem pontos de congestionamento do sistema mais efetivamente. São as chamadas rotas dispersas.

No trabalho indicado foram apresentadas aproximações que descrevem o comportamento do sistema que se utiliza desse algoritmo e também uma análise de seu desempenho, considerando

- Efeitos resultantes de situações onde os servidores se aglutinam;

- Como as rotas dispersas aliviam o problema da aglutinação e melhoram o desempenho.

O trabalho conclui que, de modo geral, o algoritmo serviço cíclico pode fornecer um bom desempenho, mesmo com não homogeneidade de cargas, a um nível de informação razoavelmente baixo.

\subsection{Conclusão}

Para uma classe de sistemas distribuídos, onde é considerado um meio de comunicação capaz apenas de transportar mensagens, os seguintes pontos foram indicados:

- A escolha de uma estratégia de load sharing é uma decisão crítica no projeto de um sistema distribuído;

- As estratégias iniciativa do servidor aparentam ter desempenho melhor que as estratégias iniciativa da origem com mesmo nível de informação;

- Quanto maior o nível de informação de uma estratégia melhor é o seu desempenho, se não levarmos em consideração na medida de desempenho o 'overhead' adicional 
em controle e comunicação introduzido na utilização da informação;

- O algoritmo Serviço Cíclico é bastante interessante, uma vez que pode fornecer um bom desempenho e envolve um 'overhead' devido a comunicação razoavelmente baixo.

Além disso, apresentamos duas críticas à medida de desempenho $Q_{A}$ proposta em [29]: a nâo praticidade do $\sup _{\lambda_{i}}$ e a dificuldade no cômputo do denominador.

Estes dois pontos nos levaram a estudar a influência da distribuição da carga entre as origens no desempenho do sistema e, além disso, como obter bons projetos e boas regras de gerência para dados algoritmos. Com esta perspectiva estudamos o algoritmo Partição dos Servidores, objeto dos próximos capítulos, que mostrou ser bastante afetado pela variação da distribuição da carga. Como decorrência desses estudos, discutimos também distribuição versus concentração de capacidades de atendimento nos servidores, em condições de custo linear. 


\section{Capítulo 3}

\section{Análise do Algoritmo Partição dos Servidores}

No capítulo anterior foram apresentados os algoritmos canônicos de load sharing. Este capítulo tem por objetivo estudar o mais simples dos algoritmos canônicos, que é o algoritmo partição dos servidores.

O algoritmo partição dos servidores (PS) consiste basicamente em dividir os servidores em tantos grupos quantas forem as origens de entrada de jobs (obviamente é suposto que o número de servidores é maior ou igual ao número de origens) e associar cada grupo a uma origem, de forma que:

- a uma origem podem estar associados vários servidores;

- um servidor só pode estar associado a uma origem.

A associação entre as origens e os servidores é feita a priori e não é mais alterada. Uma vez definida esta associação, a designação de um job a um servidor depende unicamente dela, ou seja, a definição de qual servidor vai processar o job só depende de sua origem, porisso diz-se que o algoritmo PS é de nível 1 de informação.

A distribuição dos jobs entre os servidores é feita baseada nesta associação fixa entre as origens e servidores, ou seja, existe uma regra fixa para distribuição dos jobs: um job que entra na origem $i$ é processado por um dos servidores associados à origem $i$. Porisso 
o algoritmo PS é dito estático.

O esquema geral de funcionamento do algoritmo PS é o seguinte: quando um servidor fica livre (termina o processamento de um job), ele toma para processamento o job que chegou há mais tempo na origem associada a ele. Portanto é um algoritmo com iniciativa do servidor ('server goes looking for work').

O objetivo principal do estudo desse algoritmo é a análise do seu desempenho, considerando os vários fatores que a influenciam. Boa parte desse capítulo é dedicada ao estudo da condição particular em que há tantas origens quantos servidores.

\section{1 - Formulação do algoritmo}

Uma partição de servidores para $N$ origens e $K$ servidores $(N \leq K)$, denotada por $P_{N, K}$, é definida por $N$ grupos de servidores:

$P_{N, K}=\left\{I_{1}, I_{2}, \ldots, I_{N}\right\}$

onde $I_{j}=$ conjunto que define os servidores do grupo $j$ $\equiv\{\ell \in\{1,2, \ldots, K\} \mid \ell$-ésimo servidor está associado à origem $j\}$

para $j=1,2, \ldots, N$

Define-se $c_{j}=$ número de servidores no grupo $j(\geq 1) \equiv \sharp I_{j} \quad$ para $j=1,2, \ldots, N$ Claramente $\sum_{j=1}^{N} c_{j}=K$

Serão assumidas as seguintes hipóteses:

- A entrada de jobs no sistema, via uma origem $j$, forma um processo Poisson com taxa média $\lambda_{j}$. Obviamente está implícita a hipótese $\lambda_{j}>0$, mas para estudos de casos limites admitiremos a possibilidade do aparente contrasenso prático $\lambda_{j}=0$. Temos ainda $\sum_{j=1}^{N} \lambda_{j}=\lambda$.

- A distribuição de probabilidade do tempo de atendimento de um servidor $\ell$ do sistema é exponencial, com média $\mu_{\ell}^{-1}$. Mais ainda, suporemos $\sum_{\ell=1}^{K} \mu_{\ell}=\mu$, consistente com a hipótese de custos lineares de capacidade de atendimento.

- Uma vez que o algoritmo é iniciativa do servidor, existe uma tendência para formação de filas de jobs nas origens, sendo uma fila em cada origem. A disciplina de atendimento em cada uma das filas é a FCFS. 
Assim, podemos dizer que na origem $j$ existe a formação de uma fila do tipo $M / M / c_{j}$.

Podemos também considerar o grupo $j$ correspondente a um subsistema do tipo $S\left(\lambda_{j}, \sum_{\ell \in I_{j}} \mu_{\ell}, 1, c_{j}\right)$. Desta forma, o sistema $S(\lambda, \mu, N, K)$ é constituído dos $N$ subsistemas $S\left(\lambda_{j}, \sum_{\ell \in I_{j}} \mu_{\ell}, 1, c_{j}\right), j=1, \ldots, N /$.

Seja o seguinte exemplo esquema de uma partição de servidores com $N=3$ e $K=7$ $\left(P_{3,7}\right)$.

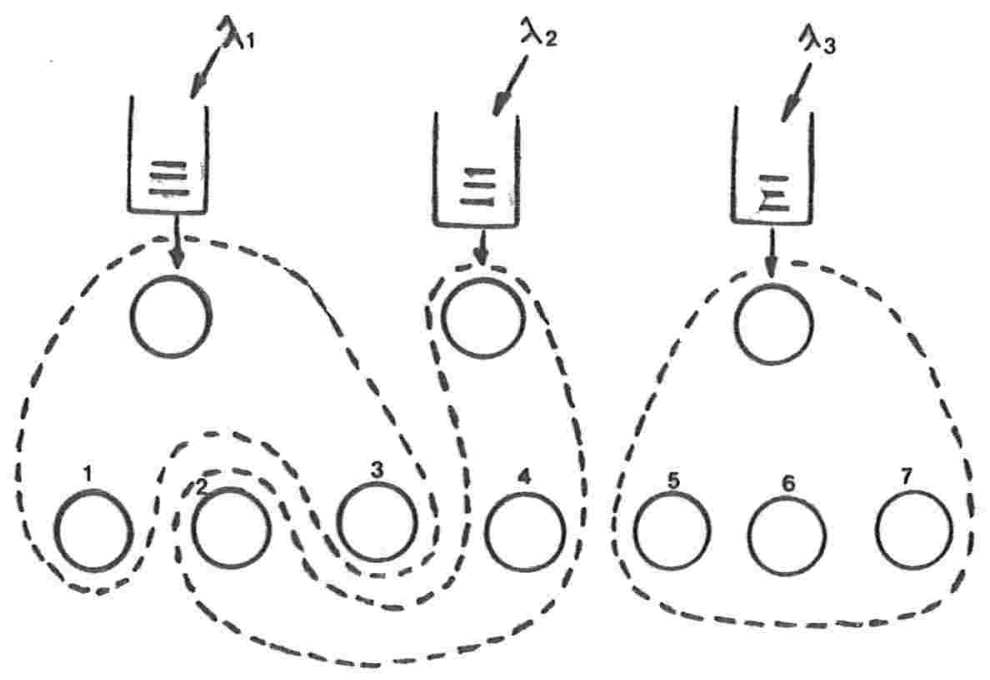

A partição acima gerou 3 grupos, sendo

$$
\begin{gathered}
I_{1}=\{1,3\}, \quad c_{1}=2 \\
I_{2}=\{2,4\}, \quad c_{2}=2 \\
I_{3}=\{5,6,7\}, \quad c_{3}=3 .
\end{gathered}
$$

A fila da origem 1 e a fila da origem 2 são ambas $M / M / 2$ e a fila da origem 3 é $M / M / 3$.

Não há perda da generalidade em assumir uma renumeração de servidores de forma que:

$$
I_{j}=\left\{i \in\{1,2, \ldots, K\} \mid \sum_{\ell=1}^{j-1} c_{\ell}<i \leq \sum_{\ell=1}^{j} c_{\ell}\right\}, \quad \text { com } \quad c_{o}=0 .
$$

No exemplo acima teríamos que renumerar os servidores 2 e 3 , de forma a obter $I_{1}=\{1,2\},, I_{2}=\{3,4\}$ e $I_{3}=\{5,6,7\}$.

A medida de desempenho utilizada para análise do algoritmo PS é o tempo médio no sistema. Dado um sistema $S(\lambda, \mu, N, K)$, o tempo médio no sistema ( $\mathrm{T}$ ) é dado por

$$
T=\sum_{j=1}^{N} \frac{\lambda_{j}}{\lambda} T_{j}
$$


onde $T_{j}$ é o tempo médio no sistema para jobs que entraram pela origem $j$, ou seja, o tempo médio no subsistema correspondente ao grupo $j$.

Também é utilizada como medida de desempenho o tempo médio na fila do sistema $\left(T_{f}\right)$, dado por

$$
T_{f}=\sum_{j=1}^{N} \frac{\lambda_{j}}{\lambda} T_{f_{j}}
$$

onde $T_{f_{j}}$ é o tempo médio na fila do subsistema correspondente ao grupo $j$.

Em cada ponto do texto será identificada claramente a medida de desempenho sendo utilizada.

Uma vez fixada uma partição de servidores, podemos analisar o desempenho do algoritmo sobre 3 formas diferentes:

1a. forma - Análise de desempenho em função da variação da distribuição da carga entre as origens.

Dada uma partição e a distribuição da capacidade total entre os servidores, determinar qual a melhor distribuição da carga total entre as origens, ou seja, qual a distribuição de cargas que fornece o melhor desempenho para o sistema.

Para esta forma de análise associaremos o $\lambda$-problema definido a seguir:

Dados um sistema $S(\lambda, \mu, N, K)$

$$
\begin{aligned}
& \mathrm{e} \quad \mu_{1}, \mu_{2}, \ldots, \mu_{K}, \quad \mu_{j} \geq 0 \quad j=1,2, \ldots, K \quad \text { e } \quad \sum_{j=1}^{K} \mu_{j}=\mu \\
& \min \quad T\left(\lambda_{1}, \lambda_{2}, \ldots, \lambda_{N}\right) \\
& \text { sujeito a } \quad \sum_{i=1}^{N} \lambda_{i}=\lambda \\
& \frac{\lambda_{i}}{\sum_{j \in I_{i}}^{\mu_{j}}}<1 \quad i=1,2, \ldots, N \\
& \lambda_{i} \geq 0 \quad i=1,2, \ldots, N .
\end{aligned}
$$

O segundo conjunto de restrições serve para garantir a não saturação de cada grupo de servidores.

A solução ótima do problema acima será denotada por $\lambda_{1}^{*}, \lambda_{2}^{*}, \ldots, \lambda_{N}^{*}$.

2a. forma - Análise de desempenho em função da distribuição da capacidade entre os servidores. 
Dada uma partição e a distribuição da carga total entre as origens, determinar qual a melhor distribuição da capacidade total entre os servidores, ou seja, qual a distribuição de capacidades que fornece o melhor desempenho para o sistema. Para esta forma de análise associaremos o $\mu$-problema definido a seguir:

Dados um sistema $S(\lambda, \mu, N, K)$

e $\quad \lambda_{1}, \lambda_{2}, \ldots, \lambda_{N} \quad$ tais que $\quad \lambda_{i} \geq 0 \quad i=1,2, \ldots, N \quad$ e $\sum_{i=1}^{N} \lambda_{i}=\lambda$ $\min T\left(\mu_{1}, \mu_{2}, \ldots, \mu_{K}\right)$

sujeito a $\quad \sum_{j=1}^{K} \mu_{j}=\mu$

$$
\begin{gathered}
\frac{\lambda_{i}}{\sum_{j \in I_{i}} \mu_{j}}<1 \quad i=1,2, \ldots, N \\
\mu_{j} \geq 0 \quad j=1,2, \ldots, N
\end{gathered}
$$

Valem aqui as mesmas observações feitas para o $\lambda$-problema.

A solução ótima para o problema acima definido será denotada por $\mu_{1}^{*}, \mu_{2}^{*}, \ldots, \mu_{K}^{*}$.

3a. forma - Análise de desempenho em função da variação da distribuição da carga entre as origens e da variação da distribuição da capacidade entre os servidores.

Dada uma partição, determinar qual a melhor distribuição da carga total entre as origens e da capacidade total entre os servidores, ou seja, qual a distribuição de cargas e capacidades fornece o melhor desempenho para o sistema.

Para essa forma de análise associaremos o $(\lambda, \mu)$-problema definido a seguir:

Dado um sistema $S(\lambda, \mu, N, K)$

$$
\begin{aligned}
\min & T\left(\lambda_{1}, \lambda_{2}, \ldots, \lambda_{N}, \mu_{1}, \mu_{2}, \ldots, \mu_{K}\right) \\
\text { sujeito a } & \sum_{i=1}^{N} \lambda_{i}=\lambda \\
& \sum_{j=1}^{K} \mu_{j}=\mu \\
& \frac{\lambda_{i}}{\sum_{j \in I_{i}}{ }^{\mu_{j}}}<1 \quad i=1, \ldots, N \\
& \lambda_{i} \geq 0 \quad i=1, \ldots, N \\
& \mu_{j} \geq 0 \quad j=1,2, \ldots, K .
\end{aligned}
$$

Valem aqui as mesmas observações feitas para o $\lambda$-problema.

A solução ótima para o problema acima definido será denotada por $\lambda_{1}^{*}, \lambda_{2}^{*}, \ldots, \lambda_{N}^{*}, \mu_{1}^{*}, \mu_{2}^{*}, \ldots, \mu_{K}^{*}$. 
Uma forma ainda mais geral de análise do algoritmo PS consistiria em determinar também qual a partição fornece o melhor desempenho para o sistema. Essa forma mais genérica é mais difícil de ser analisada.

Este capítulo vai apresentar, basicamente, a análise do algoritmo PS para $N=K$, que considera a partição de servidores composta por $N$ grupos com apenas 1 servidor $\left(c_{j}=1, j=1,2, \ldots, N\right)$, a que chamaremos de partição singular. Para este caso particular, podemos dizer que o algoritmo partição dos servidores é equivalente ao algoritmo partição das origens.

\section{2 - Análise do algoritmo para $N=K$}

Este caso particular considera grupos de apenas um servidor.

A análise é feita utilizando-se a medida de desempenho tempo médio no sistema $(\mathrm{T})$, que conforme já descrito, é dado por

$$
T=\sum_{j=1}^{N} \frac{\lambda_{j}}{\lambda} T_{j}
$$

onde $T_{j}$ é dado pelo tempo médio no sistema $M / M / 1$.

Portanto,

$$
T=\sum_{j=1}^{N} \frac{\lambda_{j}}{\lambda} \frac{1}{\mu_{j}-\lambda_{j}}=\frac{1}{\lambda} \sum_{j=1}^{N} \frac{\lambda_{j}}{\mu_{j}-\lambda_{j}}
$$

Para este caso particular, vale que a carga na origem $i$, associada ao conjunto de jobs que entram através desta origem, vai ser processada apenas pelo servidor $i$. Assim, vamos ao longo do texto, usar a expressão 'carga no servidor $i$ ', denotando a carga que entra pela origem $i$ e vai ser processada pelo correspondente servidor. 


\subsection{1 - Análise do $\lambda$-problema}

Problema da alocação ótima de cargas nas origens

\subsubsection{1 - Análise para $\mathrm{N}=\mathrm{K}=2$}

Para este caso particular, o $\lambda$-problema reduz-se ao seguinte problema, a que chamare$\operatorname{mos} P_{o}$ :

Dados $\mu_{1}, \mu_{2}>0$

$$
\begin{array}{ll}
P_{o}: \quad \min & T\left(\lambda_{1}, \lambda_{2}\right) \\
\text { sujeito a } & \lambda_{1}+\lambda_{2}=\lambda \\
& 0 \leq \lambda_{1}<\mu_{1} \\
& 0 \leq \lambda_{2}<\mu_{2}
\end{array}
$$

A análise deste caso consiste basicamente em determinar as cargas $\lambda_{1}^{*}, \lambda_{2}^{*}$, que são solução de $P_{o}$, bem como verificar o comportamento da variação das cargas ótimas em função da variação da carga total do sistema. A determinação da solução ótima de $P_{o}$ é resultante dos teoremas apresentados a seguir, sob a hipótese natural $\lambda<\mu$.

TEOREMA 3.1: $P_{o}$ tem solução ótima.

DEMONSTRAÇÃO:

\section{FATO 3.1.1:}

a) O problema $P_{o}$ é viável

b) $\exists \bar{\alpha} \in \mathbf{R}, 0<\bar{\alpha}<1$ tal que

$\forall(x, y)$ viável em $P_{o} \operatorname{com} x>\bar{\alpha} \mu_{1}$ ou $y>\bar{\alpha} \mu_{2}$

$T(x, y)>T\left(x_{0}, y_{0}\right) \quad$ onde $\left(x_{0}, y_{0}\right)$ é um ponto viável de $P_{o}$.

\section{DEMONSTRAÇÃO:}

a) Seja $x_{0}=\frac{\mu_{1}}{\mu_{1}+\mu_{2}} \lambda$ e $y_{0}=\frac{\mu_{2}}{\mu_{1}+\mu_{2}} \lambda$.

Verifica-se trivialmente que:
a.1) $x_{0} \geq 0 \quad$ e $\quad y_{0} \geq 0$
a.2) $x_{0}+y_{0}=\lambda$
a.3) $x_{0}<\mu_{1} \quad$ e $\quad y_{0}<\mu_{2}$, pois $\lambda<\mu=\mu_{1}+\mu_{2}$. 
Logo, $\left(x_{0}, y_{0}\right)$ é um ponto viável em $P_{o}$.

b) Seja $(x, y)$ viável em $P_{o}$.

b.1) Para $x>\bar{\alpha} \mu_{1}$

$$
\begin{aligned}
T(x, y) & \geq \frac{1}{\lambda} \frac{x}{\mu_{1}-x} & & \left(\text { pois } \frac{1}{\lambda} \frac{y}{\mu_{2}-y} \geq 0\right) \\
& >\frac{1}{\lambda} \frac{\mu_{1}}{\mu_{1}-\bar{\alpha} \mu_{1}} & & \left(\text { pois } \frac{x}{\mu_{1}-x} \quad \text { é estritamente crescente em } x\right) \\
& =\frac{1}{\lambda} \frac{\bar{\alpha}}{1-\bar{\alpha}} . & &
\end{aligned}
$$

Definamos então $\bar{\alpha}$ de forma que

$$
\frac{1}{\lambda} \frac{\bar{\alpha}}{1-\bar{\alpha}}=T\left(x_{0}, y_{0}\right)
$$

ou equivalentemente

$$
\bar{\alpha}=\frac{\lambda T\left(x_{0}, y_{0}\right)}{1+\lambda T\left(x_{0}, y_{0}\right)} .
$$

Obviamente $0<\bar{\alpha}<1$.

b.2) Para $y>\bar{\alpha} \mu_{2}$, de forma análoga a b.1) obtém-se o mesmo valor para $\bar{\alpha}$.

Portanto $\exists \bar{\alpha}, 0<\bar{\alpha}<1, \quad$ tal que

$\forall(x, y) \quad$ viável em $P_{o}, \operatorname{com} \quad x>\bar{\alpha} \mu_{1} \quad$ ou $\quad y>\bar{\alpha} \mu_{2}$

temos $T(x, y)>T\left(x_{0}, y_{0}\right)$.

FATO 3.1.2: $P_{o}$ pode ser reescrito conforme a seguir:

$$
\begin{array}{cl}
P_{c}: \quad \min \quad T(x, y) \\
\text { sujeito a } \quad x+y=\lambda \\
(x, y) \in V \\
V=\left\{z \in \mathbf{R}^{2} \mid 0 \leq z \leq \alpha\left(\mu_{1}, \mu_{2}\right)\right\}, \quad \text { onde } \alpha \in(0,1) .
\end{array}
$$

\section{DEMONSTRAÇÃO:}

Basta tomar $\alpha=\bar{\alpha}$ dado pelo Fato 3.1.1.

Pelo Fato 3.1.2 temos que se existir um ótimo para $P_{c}$, ele é ótimo para $P_{o}$. 
Pode-se verificar facilmente que

- $T(\cdot, \cdot)$ é uma função contínua em $V$

- $V$ é um conjunto compacto

- $B=\left\{\left(z_{1}, z_{2}\right) \in \mathbf{R}^{2} \mid z_{1}+z_{2}=\lambda\right\}$ é fechado.

Logo $B \cap V$ é compacto e $T(\cdot, \cdot)$ é contínua neste compacto, portanto $P_{c}$ tem solução ótima.

TEOREMA 3.2: $P_{o}$ pode ser resolvido considerando-se 3 problemas, $P_{x o}, P_{x y}$ e $P_{o y}$, conforme a seguir:

$$
\begin{aligned}
& P_{x o}: \quad \min T(x, 0) \\
& \text { sujeito a } x+0=\lambda \\
& 0 \leq x \leq \mu_{1} \\
& P_{x y}: \quad \min T(x, y) \\
& \text { sujeito a } x+y=\lambda \\
& (x, y) \in \chi^{o} \\
& \chi^{o}=\left\{z \in \mathbf{R}^{2} \mid 0<z<\left(\mu_{1}, \mu_{2}\right)\right\} \\
& P_{\text {oy }}: \quad \min T(0, y) \\
& \text { sujeito a } 0+y=\lambda \\
& 0 \leq y \leq \mu_{2}
\end{aligned}
$$

\section{DEMONSTRAÇÃO:}

É feita por simples verificação.

TEOREMA 3.3: Seja $\left(\lambda_{1}^{*}, \lambda_{2}^{*}\right)$ solução ótima de $P_{o}$. Se $\left(\lambda_{1}^{*}, \lambda_{2}^{*}\right) \in \chi^{o}$ então $\exists M \in \mathbf{R}$ tal que $\left(\lambda_{1}^{*}, \lambda_{2}^{*}, M\right)$ obedece Lagrange para $P_{x y}$ e então

$$
\begin{aligned}
& \lambda_{1}^{*}=\mu_{1}-(\mu-\lambda) \frac{\sqrt{\mu_{1}}}{\sqrt{\mu_{1}}+\sqrt{\mu_{2}}} \\
& \lambda_{2}^{*}=\mu_{2}-(\mu-\lambda) \frac{\sqrt{\mu_{2}}}{\sqrt{\mu_{1}}+\sqrt{\mu_{2}}}
\end{aligned}
$$




\section{DEMONSTRAÇÃO:}

As hipóteses de Lagrange verificam-se trivialmente para $P_{x y}$ :

- $\left(\lambda_{1}^{*}, \lambda_{2}^{*}\right)$ solve $P_{x y}$, pois solve $P_{o}$ e pertence a $\chi^{o}$.

- $\chi^{o}$ é aberto.

- T é uma função continuamente diferenciável.

- $h(x, y)=x+y$ é continuamente diferenciável.

- $\nabla h=(1,1)$.

Então $\exists M \in \mathbf{R}$ tal que $\left(\lambda_{1}^{*}, \lambda_{2}^{*}, M\right)$ obedece Lagrange para $P_{x y}$.

Aplicando Lagrange para $P_{x y}$ temos então

$$
\begin{aligned}
& \frac{\partial T}{\partial x}\left(\lambda_{1}^{*}, \lambda_{2}^{*}\right)=M \frac{\partial h}{\partial x}\left(\lambda_{1}^{*}, \lambda_{2}^{*}\right) \\
& \frac{\partial T}{\partial y}\left(\lambda_{1}^{*}, \lambda_{2}^{*}\right)=M \frac{\partial h}{\partial y}\left(\lambda_{1}^{*}, \lambda_{2}^{*}\right)
\end{aligned}
$$

Mas

$$
\begin{aligned}
& \frac{\partial T}{\partial x}=\frac{\mu_{1}}{\lambda\left(\mu_{1}-x\right)^{2}}>0 \quad \text { e } \quad \frac{\partial T}{\partial y}=\frac{\mu_{2}}{\lambda\left(\mu_{2}-y\right)^{2}}>0 \\
& \frac{\partial h}{\partial x}=\frac{\partial h}{\partial y}=1 .
\end{aligned}
$$

Portanto $M>0$ e podemos escrever que

$$
\lambda_{1}^{*}=\mu_{1}-\frac{\sqrt{\mu_{1}}}{\sqrt{\lambda M}} \quad \text { e } \quad \lambda_{2}^{*}=\mu_{2}-\frac{\sqrt{\mu_{2}}}{\sqrt{\lambda M}}
$$

Temos também $\lambda_{1}^{*}+\lambda_{2}^{*}=\lambda$, logo

$$
\begin{gathered}
\mu_{1}-\frac{\sqrt{\mu_{1}}}{\sqrt{\lambda M}}+\mu_{2}-\frac{\sqrt{\mu_{2}}}{\sqrt{\lambda M}}=\lambda \\
\Longleftrightarrow \frac{1}{\sqrt{\lambda M}}=\frac{\mu-\lambda}{\sqrt{\mu_{1}}+\sqrt{\mu_{2}}}
\end{gathered}
$$

E aplicando (2) em (1)

$$
\begin{aligned}
& \lambda_{1}^{*}=\mu_{1}-(\mu-\lambda) \frac{\sqrt{\mu_{1}}}{\sqrt{\mu_{1}}+\sqrt{\mu_{2}}} \quad \mathrm{e} \\
& \lambda_{2}^{*}=\mu_{2}-(\mu-\lambda) \frac{\sqrt{\mu_{2}}}{\sqrt{\mu_{1}}+\sqrt{\mu_{2}}}
\end{aligned}
$$

COROLÁRIO 3.4: Se $\nexists\left(\lambda_{1}^{*}, \lambda_{2}^{*}, M\right)$ que obedece Lagrange para $P_{x y}$ então a solução ótima de $P_{o}$ é dada pela solução de $P_{x o}$ ou pela solução de $P_{o y}$. 


\section{DEMONSTRAÇÃO:}

Imediata.

TEOREMA 3.5: $\mathrm{Se}\left(\lambda_{1}^{*}, \lambda_{2}^{*}\right)$ é viável em $P_{x y}$ e $\exists \bar{M}>0$ tal que $\left(\lambda_{1}^{*}, \lambda_{2}^{*}, \bar{M}\right)$ obedece Lagrange para $P_{x y}$, então $\left(\lambda_{1}^{*}, \lambda_{2}^{*}\right)$ solve $P_{x y}$.

\section{DEMONSTRAÇÃO:}

Seja $L$, o Lagrangeano definido para $P_{x y}$ :

$$
L(x, y, M)=\frac{1}{\lambda}\left(\frac{x}{\mu_{1}-x}+\frac{y}{\mu_{2}-y}\right)+M(x+y-\lambda) \quad \forall(x, y) \in \chi^{0}, M \in \mathbf{R}
$$

Obviamente $L(\cdot, \cdot, M)$ é uma função convexa em $\chi^{\circ}$, pois é soma de função convexas.

Temos que $L(x, y, M)=T(x, y), \forall(x, y) \in \chi^{o}$.

Assim, $\forall\left(\lambda_{1}, \lambda_{2}\right) \in \chi^{\circ}$

$$
\begin{aligned}
T\left(\lambda_{1}, \lambda_{2}\right)= & L\left(\lambda_{1}, \lambda_{2}, \bar{M}\right) \\
\geq & L\left(\lambda_{1}^{*}, \lambda_{2}^{*}, \bar{M}\right)+<\nabla L\left(\lambda_{1}^{*}, \lambda_{2}^{*}, \bar{M}\right),\left(\lambda_{1}, \lambda_{2}\right)-\left(\lambda_{1}^{*}, \lambda_{2}^{*}\right)> \\
& \quad\left(\text { pois } \quad L(\cdot, \cdot, \bar{M}), \quad \text { é convexa em } \chi^{o}\right) \\
= & L\left(\lambda_{1}^{*}, \lambda_{2}^{*}, \bar{M}\right) \quad\left(\text { pois } \quad\left(\lambda_{1}^{*}, \lambda_{2}^{*}, \bar{M}\right) \quad \text { obedece Lagrange }\right) \\
= & T\left(\lambda_{1}^{*}, \lambda_{2}^{*}\right) \quad\left(\text { pois } \quad\left(\lambda_{1}^{*}, \lambda_{2}^{*}\right) \in \chi^{o}\right)
\end{aligned}
$$

Logo $\left(\lambda_{1}^{*}, \lambda_{2}^{*}\right)$ solve $P_{x y}$.

TEOREMA 3.6: Seja $\mu_{1}>\mu_{2}$, a solução de $P_{o y}$ nunca é solução para $P_{o}$.

\section{DEMONSTRAÇÃO:}

Se $P_{o y}$ é inviável o teorema está demonitrado.

Supondo então $P_{o y}$ viável, existe apenas um ponto viável pära $P_{o y}$, logo este ponto é solução de $P_{\text {oy }}$, e o valor ótimo é dado por

$$
\frac{1}{\mu_{2}-\lambda}
$$

A solução ótima de $P_{o}$, dada por $\left(\lambda_{1}^{*}, \lambda_{2}^{*}\right)$ é da forma

$$
\lambda_{1}^{*}=p \lambda \quad \text { e } \quad \lambda_{2}^{*}=(1-p) \lambda, \quad \text { para algum } \quad p \in[0,1] .
$$


O valor ótimo da função objetivo $T$, pode ser dado pela função $T_{p}$ definida por:

$$
T_{p}(p)=\frac{p}{\mu_{1}-p \lambda}+\frac{1-p}{\mu_{2}-(1-p) \lambda} \quad p \in[0,1] .
$$

Vamos então mostrar que:

$$
\begin{aligned}
& \left.\left.T_{p}(p)<\frac{1}{\mu_{2}-\lambda}, \quad \forall p \in\right] 0,1\right] . \\
\Longleftrightarrow & \frac{p}{\mu_{1}-p \lambda}+\frac{1-p}{\mu_{2}-(1-p) \lambda}<\frac{1}{\mu_{2}-\lambda} \\
\Longleftrightarrow & p\left(\mu_{2}-(1-p) \lambda\right)\left(\mu_{2}-\lambda\right)+(1-p)\left(\mu_{1}-p \lambda\right)\left(\mu_{2}-\lambda\right)<\left(\mu_{1}-p \lambda\right)\left(\mu_{2}-(1-p) \lambda\right) \\
\Longleftrightarrow & \left(\mu_{2}-\lambda\right)\left[p\left(\mu_{2}-(1-p) \lambda\right)+(1-p)\left(\mu_{1}-p \lambda\right)-\left(\mu_{1}-p \lambda\right)\right]<p \lambda\left(\mu_{1}-p \lambda\right) \\
\Longleftrightarrow & \left(\mu_{2}-\lambda\right) p\left[\mu_{2}-(1-p) \lambda-\left(\mu_{1}-p \lambda\right)\right]<p \lambda\left(\mu_{1}-p \lambda\right) \\
\Longleftrightarrow & \left(\mu_{2}-\lambda\right)\left[\mu_{2}-\lambda+p \lambda-\left(\mu_{1}-p \lambda\right)\right]<\lambda\left(\mu_{1}-p \lambda\right) \quad(\text { pois } \quad p>0) \\
\Longleftrightarrow & \left(\mu_{2}-\lambda\right)^{2}+\left(\mu_{2}-\lambda\right) p \lambda-\mu_{2}\left(\mu_{1}-p \lambda\right)<0 \\
\Longleftrightarrow & \left(\mu_{2}-\lambda\right)^{2}-\mu_{2} \mu_{1}+p \lambda\left(\mu_{2}-\lambda+\mu_{2}\right)<0 \\
\Longleftrightarrow & p<\frac{\mu_{2} \mu_{1}-\left(\mu_{2}-\lambda\right)^{2}}{\left(2 \mu_{2}-\lambda\right) \lambda} \quad\left(p o i s \quad \lambda<\mu_{2} \quad \text { e } \quad \lambda>0\right) \\
\Longleftrightarrow & p<\frac{\mu_{2} \mu_{1}-\left(\mu_{2}^{2}-2 \mu_{2} \lambda+\lambda^{2}\right)}{\left(2 \mu_{2}-\lambda\right) \lambda}=\frac{\mu_{2}\left(\mu_{1}-\mu_{2}\right)+\left(2 \mu_{2}-\lambda\right) \lambda}{\left(2 \mu_{2}-\lambda\right) \lambda} \\
\Longleftrightarrow & p<1+\frac{\mu_{2}\left(\mu_{1}-\mu_{2}\right)}{\left(2 \mu_{2}-\lambda\right) \lambda} \\
\mathrm{Mas} & \mu_{1}>\mu_{2} \quad \mathrm{e} \quad \lambda<\mu_{2} \Longleftrightarrow \frac{\mu_{2}\left(\mu_{1}-\mu_{2}\right)}{\left(2 \mu_{2}-\lambda\right) \lambda}>0
\end{aligned}
$$

Logo,

$$
\left.\left.T_{p}(p)<\frac{1}{\mu_{2}-\lambda}, \quad \forall p \in\right] 0,1\right]
$$

TEOREMA 3.7: Se $\mu_{1}>\mu_{2}$, então existe $\bar{\lambda}, 0<\bar{\lambda}<\mu$, tal que se $\lambda \leq \bar{\lambda}$, então a solução ótima de $P_{o}$ é dada pela solução de $P_{x o}$ se $\lambda \in] \bar{\lambda}, \mu\left[\right.$ então a solução ótima de $P_{o}$ é dada pela solução de $P_{x y}$. 


\section{DEMONSTRAÇÃO:}

A solução de $P_{x y}$, se existir, é dada por $\left(\lambda_{1}^{*}, \lambda_{2}^{*}\right)$, com

$$
\lambda_{1}^{*}=\mu_{1}-(\mu-\lambda) \frac{\sqrt{\mu_{1}}}{\sqrt{\mu_{1}}+\sqrt{\mu_{2}}} \quad \text { e } \quad \lambda_{2}^{*}=\mu_{2}-(\mu-\lambda) \frac{\sqrt{\mu_{2}}}{\sqrt{\mu_{1}}+\sqrt{\mu_{2}}} .
$$

$\operatorname{Temos} \lambda_{1}^{*}<\mu_{1}$ e $\lambda_{2}^{*}<\mu_{2}$

$$
\begin{aligned}
\lambda_{1}^{*}<0 & \Longleftrightarrow \mu_{1}-(\mu-\lambda) \frac{\sqrt{\mu_{1}}}{\sqrt{\mu_{1}}+\sqrt{\mu_{2}}}<0 \\
& \Longleftrightarrow \mu_{1}+\sqrt{\mu_{1}} \sqrt{\mu_{2}}-\left(\mu_{1}+\mu_{2}-\lambda\right)<0 \\
& \Longleftrightarrow \lambda<\mu_{2}-\sqrt{\mu_{1}} \sqrt{\mu_{2}}=\bar{\lambda}_{1}^{*} .<0
\end{aligned}
$$

Analogamente

$$
\lambda_{2}^{*}<0 \Longleftrightarrow \lambda<\mu_{1}-\sqrt{\mu_{1}} \sqrt{\mu_{2}}=\bar{\lambda}_{2}^{*}
$$

$\mathrm{E} \bar{\lambda}_{2}^{*}>\bar{\lambda}_{1}^{*}$ pois $\mu_{1}>\mu_{2}$.

Seja então $\bar{\lambda}=\bar{\lambda}_{2}^{*}$.

$$
\begin{aligned}
& \bar{\lambda}=\mu_{1}-\sqrt{\mu_{1}} \sqrt{\mu_{2}}=\sqrt{\mu_{1}}\left(\sqrt{\mu_{1}}-\sqrt{\mu_{2}}\right)>0 \\
& \bar{\lambda}=\mu_{1}-\sqrt{\mu_{1}} \sqrt{\mu_{2}}=\mu-\left(\mu_{2}+\sqrt{\mu_{1}} \sqrt{\mu_{2}}\right)<\mu .
\end{aligned}
$$

Se $\lambda \leq \bar{\lambda}$ então o ponto $\left(\lambda_{1}^{*}, \lambda_{2}^{*}\right)$ obtido pelas condições de Lagrange para $P_{x y}$, não é viável para este problema e pelo corolário 3.4 e pelo teorema 3.6 temos que a solução de $P_{o}$ é dada pela solução de $P_{x o}$, que é $(\lambda, 0)$.

Se $\lambda>\bar{\lambda}$ então temos que $\left(\lambda_{1}^{*}, \lambda_{2}^{*}\right)$ é viável e obedece Lagrange para $P_{x y}$, e portanto pelo teorema 3.5 temos que é solução para $P_{x y}$.

Vamos então mostrar que $\left(\lambda_{1}^{*}, \lambda_{2}^{*}\right)$ é melhor que $(\lambda, 0)$.

O valor da função objetivo $T\left(\lambda_{1}^{*}, \lambda_{2}^{*}\right)$ pode ser escrito por $T_{p}$ :

$$
T_{p}=\frac{p}{\mu_{1}-p \lambda}+\frac{1-p}{\mu_{2}-(1-p) \lambda}
$$

para $p \in] 0,1\left[\right.$ tal que $\lambda_{1}^{*}=p * \lambda \quad$ e $\quad \lambda_{2}^{*}=(1-p) \lambda$. 
Basta mostrar que

$$
\begin{aligned}
& T_{p}<\frac{1}{\mu_{1}-\lambda} \quad \text { para } \lambda<\bar{\lambda} \\
\Longleftrightarrow & \frac{p}{\mu_{1}-p \lambda}+\frac{1-p}{\mu_{2}-(1-p) \lambda}<\frac{1}{\mu_{1}-\lambda} \\
\Longleftrightarrow & p\left[\left(\mu_{2}-(1-p) \lambda\right]\left(\mu_{1}-\lambda\right)+(1-p)\left(\mu_{1}-p \lambda\right)\left(\mu_{1}-\lambda\right)\right. \\
& <\left(\mu_{1}-p \lambda\right)\left[\mu_{2}-(1-p) \lambda\right] \\
\Longleftrightarrow & p \mu_{2}\left(\mu_{1}-\lambda\right)-p(1-p) \lambda\left(\mu_{1}-\lambda\right)+(1-p)\left(\mu_{1}-p \lambda\right)\left(\mu_{1}-\lambda\right) \\
& <\mu_{1} \mu_{2}-\lambda \mu_{1}(1-p)-p \lambda \mu_{2}+p(1-p) \lambda^{2} \\
\Longleftrightarrow & -p(1-p) \lambda \mu_{1}+(1-p)\left(\mu_{1}-p \lambda\right)\left(\mu_{1}-\lambda\right)<(1-p) \mu_{1} \mu_{2}-\lambda \mu_{1}(1-p) \\
\Longleftrightarrow & (1-p) \lambda \mu_{1}+\left(\mu_{1}-p \lambda\right)\left(\mu_{1}-\lambda\right)<\mu_{1} \mu_{2} \\
\Longleftrightarrow & \lambda \mu_{1}-p \lambda \mu_{1}+\mu_{1}^{2}-\mu_{1} \lambda-p \lambda \mu_{1}+p \lambda^{2}<\mu_{1} \mu_{2} \\
\Longleftrightarrow & \mu_{1}^{2}-2 p \lambda \mu_{1}+p \lambda^{2}-\mu_{1} \mu_{2}<0
\end{aligned}
$$

Seja então $F(p)=\mu_{1}^{2}-2 p \lambda \mu_{1}+p \lambda^{2}-\mu_{1} \mu_{2}=p\left(\lambda^{2}-2 \lambda \mu_{1}\right)+\left(\mu_{1}^{2}-\mu_{1} \mu_{2}\right)$

Vamos analisar o sinal da função $F$ :

i) $F(0)=\mu_{1}^{2}-\mu_{1} \mu_{2}=\mu_{1}\left(\mu_{1}-\mu_{2}\right)>0 \quad$ pois $\mu_{1}<\mu_{2}$

ii) $F(1)=\lambda^{2}-2 \lambda \mu_{1}+\mu_{1}^{2}-\mu_{1} \mu_{2}=G(\lambda)$

$G(\lambda)=0 \quad$ para $\quad \lambda_{1}=\mu_{1}-\sqrt{\mu_{1}} \sqrt{\mu_{2}}=\bar{\lambda} \quad$ e $\quad \lambda_{2}=\mu_{1}+\sqrt{\mu_{1}} \sqrt{\mu_{2}}>\mu_{1}+\mu_{2}=\mu$

$G(\lambda) \geq 0 \quad$ para $\quad \lambda \leq \bar{\lambda}$ e $\lambda \geq \lambda_{2}$

$G(\lambda)<0 \quad$ para $\quad \bar{\lambda}<\lambda<\lambda_{2}$

Portanto $\lambda>\bar{\lambda} \Rightarrow F(1)<0$.

iii) $F(\bar{p})=0 \Rightarrow \bar{p}=\frac{\mu_{1}\left(\mu_{1}-\mu_{2}\right)}{\lambda\left(2 \mu_{1}-\lambda\right)}$

Seja

$$
p^{*}=\frac{\lambda_{1}^{*}}{\lambda}=\frac{1}{\lambda}\left(\mu_{1}-(\mu-\lambda) \frac{\sqrt{\mu_{1}}}{\sqrt{\mu_{1}} \sqrt{\mu_{2}}}\right)=\frac{1}{\lambda}\left(\mu_{1}-(\mu-\lambda) \frac{\left(\mu_{1}-\sqrt{\mu_{1}} \sqrt{\mu_{2}}\right)}{\mu_{1}-\mu_{2}}\right) .
$$

Para provar que $F\left(p^{*}\right)<0$, pelos resultados (A), (B) e (C), basta provar que $\bar{p}<p^{*}$. 
Seja então

$$
\begin{aligned}
G & =\left(\bar{p}-p^{*}\right) * \lambda \\
& =\frac{\mu_{1}\left(\mu_{1}-\mu_{2}\right)}{2 \mu_{1}-\lambda}-\frac{\mu_{1}\left(\mu_{1}-\mu_{2}\right)-(\mu-\lambda)\left(\mu_{1}-\sqrt{\mu_{1}} \sqrt{\mu_{2}}\right)}{\mu_{1}-\mu_{2}} \\
& =\frac{\mu_{1}\left(\mu_{1}-\mu_{2}\right)\left(\mu_{1}-\mu_{2}\right)-\mu_{1}\left(\mu_{1}-\mu_{2}\right)\left(2 \mu_{1}-\lambda\right)+\left(2 \mu_{1}-\lambda\right)(\mu-\lambda)\left(\mu_{1}-\sqrt{\mu_{1}} \sqrt{\mu_{2}}\right)}{\left(2 \mu_{1}-\lambda\right)\left(\mu_{1}-\mu_{2}\right)} \\
& =\frac{\mu_{1}\left(\mu_{1}-\mu_{2}\right)\left(\mu_{1}-\mu_{2}-2 \mu_{1}+\lambda\right)+\left(2 \mu_{1}-\lambda\right)(\mu-\lambda)\left(\mu_{1}-\sqrt{\mu_{1}} \sqrt{\mu_{2}}\right)}{\left(2 \mu_{1}-\lambda\right)\left(\mu_{1}-\mu_{2}\right)} \\
& =\frac{(\mu-\lambda)}{\left(2 \mu_{1}-\lambda\right)\left(\mu_{1}-\mu_{2}\right)}\left(-\mu_{1}\left(\mu_{1}-\mu_{2}\right)+\left(2 \mu_{1}-\lambda\right)\left(\mu_{1}-\sqrt{\mu_{1}} \sqrt{\mu_{2}}\right)\right)
\end{aligned}
$$

Seja $H=-\mu_{1}\left(\mu_{1}-\mu_{2}\right)+\left(2 \mu_{1}-\lambda\right)\left(\mu_{1}-\sqrt{\mu_{1}} \sqrt{\mu_{2}}\right)$

temos $\sin a l(\mathrm{G})=\operatorname{sinal}(\mathrm{H})$

$$
\begin{aligned}
H & =-\mu_{1}\left(\mu_{1}-\mu_{2}\right)+2 \mu_{1}\left(\mu_{1}-\sqrt{\mu_{1}} \sqrt{\mu_{2}}\right)-\lambda\left(\mu_{1}-\sqrt{\mu_{1}} \sqrt{\mu_{2}}\right) \\
& <-\mu_{1}\left(\mu_{1}-\mu_{2}\right)+2 \mu_{1}\left(\mu_{1}-\sqrt{\mu_{1}} \sqrt{\mu_{2}}\right)-\left(\mu_{1}-\sqrt{\mu_{1}} \sqrt{\mu_{2}}\right)^{2} \quad(\text { pois } \quad \lambda<\bar{\lambda}) \\
& =-\mu_{1}^{2}+\mu_{1} \mu_{2}+2 \mu_{1}^{2}-2 \mu_{1} \sqrt{\mu_{1}} \sqrt{\mu_{2}}-\left(\mu_{1}^{2}-2 \mu_{1} \sqrt{\mu_{1}} \sqrt{\mu_{2}}+\mu_{1} \mu_{2}\right) \\
& =0
\end{aligned}
$$

Portanto $G<0$ e $\bar{p}<p^{*}$.

RESULTADO 3.8: Seja $p^{*}$ a função que dada uma carga $\lambda$, define a distribuição ótima dessa carga entre as origens, da seguinte forma:

$$
p^{*}(\lambda)=\frac{\lambda_{1}^{*}(\lambda)}{\lambda}, \quad 0<\lambda<\mu .
$$

Vale que:

a) $p^{*}(\lambda)=1, \quad 0<\lambda \leq \bar{\lambda}$

b) Se os dois servidores têm capacidades. diferentes (podemos supor, sem perda de generalidade, $\left.\mu_{1}>\mu_{2}\right)$ e $\lambda>\bar{\lambda}$ temos que:

b.1) $p^{*}(\lambda)$ é decrescente $\operatorname{com} \lambda$

b.2) $p^{*}(\lambda)$ é convexa em $\lambda$

c) $\lim _{\lambda \rightarrow \mu} p^{*}(\lambda)=\frac{\mu_{1}}{\mu}$

ou equivalentemente $\quad \rho \rightarrow 1 \Rightarrow p^{*} \rightarrow \frac{\mu_{1}}{\mu}$

d) $\lim _{\varepsilon \rightarrow 0} p^{*}(\bar{\lambda}+\varepsilon)=1$ 


\section{DEMONSTRAÇÃO:}

a) É imediato do teorema 3.7

b) Pelo teorema 3.7 temos que a distribuição ótima de carga é dada por $\lambda_{1}^{*}=\mu_{1}-(\mu-\lambda) \frac{\sqrt{\mu_{1}}}{\sqrt{\mu_{1}}+\sqrt{\mu_{2}}}$ e $\lambda_{2}^{*}=\lambda-\lambda_{1}^{*}, \quad$ para $\lambda>\bar{\lambda}$.

Logo,

$$
\begin{aligned}
p^{*}(\lambda) & =\frac{1}{\lambda}\left(\mu_{1}-(\mu-\lambda) \frac{\sqrt{\mu_{1}}}{\sqrt{\mu_{1}}+\sqrt{\mu_{2}}}\right) \\
& =\frac{1}{\lambda}\left(\mu_{1}-\mu \frac{\sqrt{\mu_{1}}}{\sqrt{\mu_{1}}+\sqrt{\mu_{2}}}\right)+\frac{\sqrt{\mu_{1}}}{\sqrt{\mu_{1}}+\sqrt{\mu_{2}}}
\end{aligned}
$$

Sejam $A=\mu_{1}-\mu \frac{\sqrt{\mu_{1}}}{\sqrt{\mu_{1}}+\sqrt{\mu_{2}}} \quad$ e $\quad B=\frac{\sqrt{\mu_{1}}}{\sqrt{\mu_{1}}+\sqrt{\mu_{2}}}$, temos

$$
p^{*}(\lambda)=\frac{A}{\lambda}+B \Rightarrow p^{*^{\prime}}(\lambda)=-\frac{A}{\lambda^{2}}
$$

b.1) Basta provar que $A \geq 0$

$$
\begin{aligned}
& A \geq 0 \\
\Longleftrightarrow & \mu_{1}-\mu \frac{\sqrt{\mu_{1}}}{\sqrt{\mu_{1}}+\sqrt{\mu_{2}}} \geq 0 \\
\Longleftrightarrow & \mu_{1}\left(\sqrt{\mu_{1}}+\sqrt{\mu_{2}}\right)-\sqrt{\mu_{1}}\left(\mu_{1}+\mu_{2}\right) \geq 0 \\
\Longleftrightarrow & \sqrt{\mu_{1}}\left(\sqrt{\mu_{1}} \sqrt{\mu_{2}}-\mu_{2}\right) \geq 0 \\
\Longleftrightarrow & \sqrt{\mu_{2}}\left(\sqrt{\mu_{1}}-\sqrt{\mu_{2}}\right) \geq 0
\end{aligned}
$$

Mas $\mu_{1}>\mu_{2}$, por hipótese, logo $A \geq 0$.

b.2) Dados $\lambda_{1}, \lambda_{2} \in \mathbf{R}^{+}, \lambda_{1} \neq \lambda_{2}$, temos

$$
\begin{aligned}
{\left[p^{*^{\prime}}\left(\lambda_{2}\right)-p^{*^{\prime}}\left(\lambda_{1}\right)\right]\left(\lambda_{2}-\lambda_{1}\right) } & =\left[-\frac{A}{\lambda_{2}^{2}}+\frac{A}{\lambda_{1}^{2}}\right]\left(\lambda_{2}-\lambda_{1}\right)=\frac{A\left(\lambda_{2}-\lambda_{1}\right)\left(-\lambda_{1}^{2}+\lambda_{2}^{2}\right)}{\lambda_{1}^{2} \lambda_{2}^{2}} \\
& =\frac{A\left(\lambda_{2}-\lambda_{1}\right)^{2}\left(\lambda_{1}+\lambda_{2}\right)}{\lambda_{1}^{2} \lambda_{2}^{2}} \geq 0
\end{aligned}
$$

Portanto $p^{*}$ é convexa em $\lambda$.

c) $\lim _{\lambda \rightarrow \mu} p^{*}(\lambda)=\lim _{\lambda \rightarrow \mu} \frac{1}{\lambda}\left(\mu_{1}-(\mu-\lambda) \frac{\sqrt{\mu_{1}}}{\sqrt{\mu_{1}}+\sqrt{\mu_{2}}}\right)=\frac{\mu_{1}}{\mu}$

d) $p^{*}(\bar{\lambda}+\varepsilon)=\frac{\lambda_{1}^{*}(\bar{\lambda}+\varepsilon)}{\bar{\lambda}+\varepsilon}=\frac{1}{\bar{\lambda}+\varepsilon}\left(\mu_{1}-(\mu-(\bar{\lambda}+\varepsilon)) \frac{\sqrt{\mu_{1}}}{\sqrt{\mu_{1}}+\sqrt{\mu_{2}}}\right)$

Portanto

$$
\begin{aligned}
\lim _{\varepsilon \rightarrow 0} p^{*} & (\bar{\lambda}+\varepsilon)=\frac{1}{\lambda}\left(\mu_{1}-(\mu-\bar{\lambda}) \frac{\sqrt{\mu_{1}}}{\sqrt{\mu_{1}}+\sqrt{\mu_{2}}}\right) \\
& =\frac{1}{\mu_{1}-\sqrt{\mu_{1}} \sqrt{\mu_{2}}}\left(\mu_{1}-\left(\mu-\mu_{1}+\sqrt{\mu_{1}} \sqrt{\mu_{2}}\right) \frac{\sqrt{\mu_{1}}}{\sqrt{\mu_{1}}+\sqrt{\mu_{2}}}\right)
\end{aligned}
$$




$$
\begin{aligned}
& =\frac{1}{\mu_{1}-\sqrt{\mu_{1}} \sqrt{\mu_{2}}}\left(\mu_{1}-\frac{\mu_{2} \sqrt{\mu_{1}}+\mu_{1} \sqrt{\mu_{2}}}{\sqrt{\mu_{1}}+\sqrt{\mu_{2}}}\right) \\
& =\frac{1}{\mu_{1}-\sqrt{\mu_{1}} \sqrt{\mu_{2}}}\left(\mu_{1}-\sqrt{\mu_{1}} \sqrt{\mu_{2}} \frac{\left(\sqrt{\mu_{2}}+\sqrt{\mu_{1}}\right)}{\sqrt{\mu_{1}}+\sqrt{\mu_{2}}}\right) \\
& =1
\end{aligned}
$$

O resultado 3.8 nos permite traçar o gráfico da razão da carga ótima no servidor 'mais forte' (mais capacidade) e a carga total em função da variação da carga total.

Neste gráfico adotamos $p^{*}(0)=1$, que pode ser entendido da seguinte forma: toda a carga (nula) é atribuída ao servidor 'mais forte'.

A partir do gráfico podemos extrair as seguintes informações:

- Para cargas baixas do sistema,o melhor desempenho pode ser obtido concentrando-se a carga em apenas um servidor.

- A carga no servidor 'mais forte' diminui à medida em que a carga total aumenta, sendo limitada inferiormente por $\frac{\mu_{1}}{\mu_{1}+\mu_{2}}$.

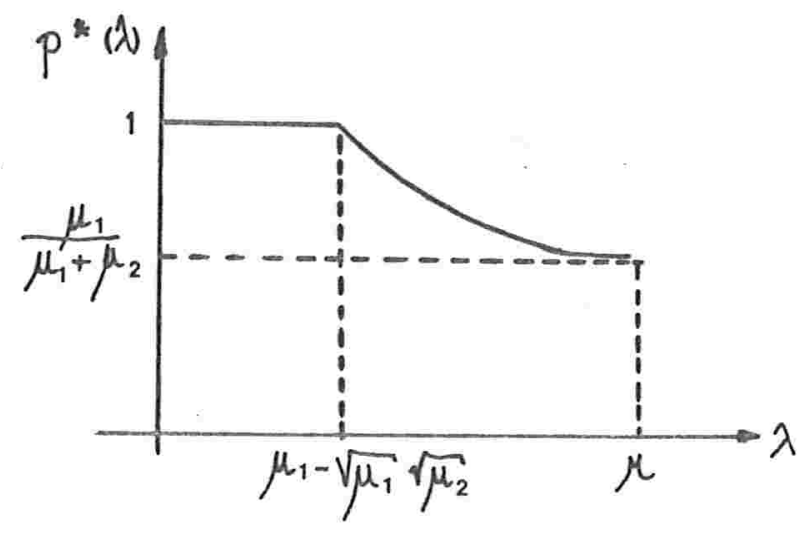

TEOREMA 3.9: Se $\mu_{1}=\mu_{2}$, então a solução de $P_{o}$ é dada pela solução de $P_{x y}$, que é definida por $\lambda_{1}^{*}=\lambda_{2}^{*}=\frac{\lambda}{2}$.

\section{DEMONSTRAÇÃO:}

Neste caso, os problemas $P_{x o}$ e $P_{o y}$ são equivalentes (mesma função objetivo e mesmo conjunto de restrições).

A solução de $P_{x y}$, se existir, é $\left(\lambda_{1}^{*}, \lambda_{2}^{*}\right)$ tal que

$$
\lambda_{i}^{*}=\mu_{i}-(\mu-\lambda) \frac{\sqrt{\mu_{i}}}{\sqrt{\mu_{1}}+\sqrt{\mu_{2}}}=\frac{\lambda}{2}>0 \quad i=1,2
$$


Logo, $\left(\lambda_{1}^{*}, \lambda_{2}^{*}\right)$ é viável para $P_{x y}$ e pelo teorema 3.5 , solve $P_{x y}$.

Para obter a solução de $P_{o}$, basta então comparar as soluções de $P_{x y}$ e $P_{x o}$.

$\mathrm{O}$ valor ótimo de $P_{x y}$ é

$$
T\left(\lambda_{1}^{*}, \lambda_{2}^{*}\right)=\frac{1}{\lambda}\left(\frac{\lambda_{1}^{*}}{\mu_{1}-\lambda_{1}^{*}}+\frac{\lambda_{2}^{*}}{\mu_{2}-\lambda_{2}^{*}}\right)=\frac{2}{\mu-\lambda}
$$

O valor ótimo de $P_{x o}$ é

$$
T(\lambda, 0)=\frac{1}{\frac{\mu}{2}-\lambda}=\frac{2}{\mu-2 \lambda}
$$

Mas

$$
\begin{aligned}
& T\left(\lambda_{1}^{*}, \lambda_{2}^{*}\right)<T(\lambda, 0) \\
\Longleftrightarrow & \frac{2}{\mu-\lambda}<\frac{2}{\mu-2 \lambda} \\
\Longleftrightarrow & \mu-\lambda>\mu-2 \lambda \\
\Longleftrightarrow & \lambda>0
\end{aligned}
$$

Portanto $\left(\lambda_{1}^{*}, \lambda_{2}^{*}\right)=\left(\frac{\lambda}{2}, \frac{\lambda}{2}\right)$ é solução de $P_{o}$.

3.2.1.2 - Análise para $\mathrm{N}=\mathrm{K}=\mathrm{m}$

Esta análise consiste basicamente em determinar as cargas $\lambda_{1}^{*}, \lambda_{2}^{*}, \ldots, \lambda_{m}^{*}$, que são a solução do seguinte problema, a que chamaremos $P_{o}$.

Dados $\mu_{1}, \mu_{2}, \ldots, \mu_{m}>0$

$$
\begin{aligned}
P_{o}: \quad \min & T\left(\lambda_{1}, \lambda_{2}, \ldots, \lambda_{m}\right) \\
\text { sujeito a } & \sum_{i=1}^{m} \lambda_{i}=\lambda \\
& 0 \leq \lambda_{i}<\mu_{i} \quad i=1,2, \ldots, m
\end{aligned}
$$

Esta solução será obtida de forma análoga ao caso $m=2$, através de generalização de alguns dos resultados lá demonstrados.

TEOREMA 3.10: $P_{o}$ tem solução ótima.

\section{DEMONSTRAÇÃO:}

Será omitida por ser análoga à do Teorema 3.1. 
TEOREMA 3.11: $P_{o}$ pode ser resolvido recursivamente considerando $(m+1)$ problemas, $P_{1}, P_{2}, \ldots, P_{m}$ e $P_{x}$, conforme a seguir

$$
\begin{aligned}
P_{j}: \quad \min \quad & T\left(x_{1}, \ldots, x_{j}, \ldots, x_{m}\right) \\
\text { sujeito a } \quad x_{j}=0 & \\
& \sum_{i=1}^{m} x_{i}=\lambda \\
& 0 \leq x_{i} \leq \mu_{i} \quad i=1,2, \ldots, m \\
j=1,2, \ldots, m & \\
P_{x}: \quad & T\left(x_{1}, x_{2}, \ldots, x_{m}\right) \\
\text { min } \quad \text { sujeito a } \quad & \sum_{i=1}^{m} x_{i}=\lambda \\
& \left(x_{1}, x_{2}, \ldots, x_{m}\right) \in \chi^{o} \\
& \chi^{o}=\left\{z \in \mathbf{R}^{m} \mid 0<z<\left(\mu_{1}, \mu_{2}, \ldots, \mu_{m}\right)\right\}
\end{aligned}
$$

\section{DEMONSTRAÇÃO:}

É feita por simples verificação, observando que para resolver qualquer $P_{i}$ temos que resolver recursivamente $(m-1)$ problemas derivados analogamente.

T⿱EOREMA 3.12: Seja $\left(\lambda_{1}^{*}, \lambda_{2}^{*}, \ldots, \lambda_{m}^{*}\right)$ solução de $P_{o}$. Se $\left(\lambda_{1}^{*}, \lambda_{2}^{*}, \ldots, \lambda_{m}^{*}\right) \in \chi^{o}$, então $\exists M \in \mathbf{R}$ tal que $\left(\lambda_{1}^{*}, \lambda_{2}^{*}, \ldots, \lambda_{m}^{*}, M\right)$ obedece Lagrange e então

$$
\lambda_{i}^{*}=a_{i} * \lambda+b_{i} \quad i=1,2, \ldots, m
$$

onde

$$
\begin{aligned}
& a_{i}=\frac{\sqrt{\mu_{i}}}{\sum_{j=1}^{m} \sqrt{\mu_{j}}} \\
& b_{i}=\mu_{i}-\mu \frac{\sqrt{\mu_{i}}}{\sum_{j=1}^{m} \sqrt{\mu_{j}}}=\frac{\mu_{i} \sum_{\substack{j \neq i \\
j=1}}^{m} \sqrt{\mu_{j}}-\sqrt{\mu_{i}} \sum_{\substack{j \neq i \\
j=1}}^{m} \mu_{j}}{\sum_{j=1}^{m} \sqrt{\mu_{j}}}
\end{aligned}
$$

\section{DEMONSTRAÇÃO:}

As hipóteses de Lagrange verificam-se trivialmente para $P_{x}$ (analogamente ao caso $m=2$ ).

Então $\exists M \in \mathbf{R}$ tal que $\left(\lambda_{1}^{*}, \lambda_{2}^{*}, \ldots, \lambda_{m}^{*}\right)$ obedece Lagrange para $P_{x}$.

Por Lagrange temos então, para $i=1,2, \ldots, m$ :

$$
\frac{\partial T}{\partial x_{i}}\left(\lambda_{1}^{*}, \lambda_{2}^{*}, \ldots, \lambda_{m}^{*}\right)=M * \frac{\partial h}{\partial x_{i}}\left(\lambda_{1}^{*}, \lambda_{2}^{*}, \ldots, \lambda_{m}^{*}\right) \quad \text { onde } \quad h\left(x_{1}, x_{2}, \ldots, x_{m}\right)=\sum_{j=1}^{m} x_{j}
$$


Mas

$$
\frac{\partial T}{\partial x_{i}}=\frac{\mu_{i}}{\lambda\left(\mu_{i}-x_{i}\right)^{2}}>0 \quad \text { e } \quad \frac{\partial h}{\partial x_{i}}=1
$$

Portanto $\mu>0$ e podemos escrever que

$$
\lambda_{i}^{*}=\mu_{i}-\frac{\sqrt{\mu_{i}}}{\sqrt{\lambda M}}
$$

Mas

$$
\lambda=\sum_{j=1}^{m} \lambda_{j}^{*}=\sum_{j=1}^{m}\left(\mu_{j}-\frac{\sqrt{\mu_{j}}}{\sqrt{\lambda M}}\right)
$$

Portanto

$$
\frac{1}{\sqrt{\lambda M}}=\frac{(\mu-\lambda)}{\sum_{j=1}^{m} \sqrt{\mu_{j}}}
$$

E aplicando (2) em (1)

$$
\begin{aligned}
\lambda_{i}^{*} & =\mu_{i}-\sqrt{\mu_{i}} \frac{(\mu-\lambda)}{\sum_{j=1}^{m} \sqrt{\mu_{j}}} \\
& =\frac{\sqrt{\mu_{i}}}{\sum_{j=1}^{m} \sqrt{\mu_{i}}} * \lambda+\mu_{i}-\mu \frac{\sqrt{\mu_{i}}}{\sum_{j=1}^{m} \sqrt{\mu_{j}}}
\end{aligned}
$$

Basta então definir

$$
a_{i}=\frac{\sqrt{\mu_{i}}}{\sum_{j=1}^{m} \sqrt{\mu_{j}}} \text { e } b_{i}=\mu_{i}-\mu \frac{\sqrt{\mu_{i}}}{\sum_{j=1}^{m} \sqrt{\mu_{j}}}
$$

Temos então

$$
b_{i}=\frac{\mu_{i} \sum_{j=1}^{m} \sqrt{\mu_{j}}-\mu \sqrt{\mu_{i}}}{\sum_{j=1}^{m} \sqrt{\mu_{j}}}=\frac{\mu_{i} \sum_{\substack{j \neq i \\ j=1}}^{m} \sqrt{\mu_{j}}-\sqrt{\mu_{i}} \sum_{\substack{j \neq i \\ j=1}}^{m} \mu_{j}}{\sum_{j=1}^{m} \sqrt{\mu_{j}}}
$$

\section{Interpretação da solução de Lagrange}

Se a solução do $\lambda$-problema tem a forma dada pelo teorema 3.12 , isto é,

$$
\lambda_{i}^{*}=\frac{\sqrt{\mu_{i}}}{\sum_{j=1}^{m} \sqrt{\mu_{j}}} * \lambda+\mu_{i}-\mu \frac{\sqrt{\mu_{i}}}{\sum_{j=1}^{m} \sqrt{\mu_{j}}} \quad i=1,2, \ldots, m
$$

que ainda pode ser escrita como

$$
\lambda_{i}^{*}=\mu_{i}-(\mu-\lambda) \frac{\sqrt{\mu_{i}}}{\sum_{j=1}^{m} \sqrt{\mu_{j}}}
$$


podemos dar a seguinte interpretação para a distribuição ótima da carga nas origens, adaptada a partir de [15]:

- A cada origem é atribuído o máximo fluxo de jobs possível, dado pela capacidade do servidor a ela associado.

- O excesso do fluxo de jobs $(\mu-\lambda)$ é excluído das origens, na proporção das raízes quadradas das capacidades dos servidores a elas associados.

Veremos a seguir que este tipo de solução só ocorre para cargas maiores que um certo valor limite.

COROLÁRIO 3.13: Se $\exists\left(\lambda_{1}^{*}, \lambda_{2}^{*}, \ldots, \lambda_{m}^{*}, M\right)$ que obedece Lagrange para $P_{x}$ então a solução ótima de $P_{o}$ é dada pela solução de algum dos $P i, i \in\{1,2, \ldots, m\}$.

\section{DEMONSTRAÇÃO:}

Imediata.

TEOREMA 3.14: Se $\left(\lambda_{1}^{*}, \lambda_{2}^{*}, \ldots, \lambda_{m}^{*}\right)$ é viável em $P_{x}$ e $\exists \bar{M}>0$ tal que $\left(\lambda_{1}^{*}, \lambda_{2}^{*}, \ldots, \lambda_{m}^{*}, \bar{M}\right)$ obedece Lagrange para $P_{x}$, então $\left(\lambda_{1}^{*}, \lambda_{2}^{*}, \ldots, \lambda_{m}^{*}\right)$ solve $P_{x}$.

\section{DEMONSTRAÇÃO:}

Será omitida por ser análoga à do teorema 3.5 , para o caso $m=2$.

TEOREMA 3.15: Se $\mu_{i} \geq \mu_{i+1}, i \in\{1,2, \ldots, m-1\}$ temos que valor ótimo $\left(P_{i}\right) \geq$ valor ótimo $\left(P_{i+1}\right)$ sendo que se $\mu_{i}>\mu_{i+1}$ vale que valor ótimo $\left(P_{i}\right)>$ valor ótimo $\left(P_{i+1}\right)$ e se $\mu_{i}=\mu_{i+1}$ vale que valor ótimo $\left(P_{i}\right)=$ valor ótimo $\left(P_{i+1}\right)$.

\section{DEMONSTRAÇÃO:}

Seja $A=\left(\lambda_{1}^{*}, \lambda_{2}^{*}, \ldots, \lambda_{i-1}^{*}, 0, \lambda_{i+1}^{*}, \ldots, \lambda_{m}^{*}\right)$ a. solução de $P i$, valor ótimo $(P i)=T(A)$

Tomemos $B=\left(\lambda_{1}^{*}, \ldots, \lambda_{i-1}^{*}, \lambda_{i+1}^{*}, 0, \ldots, \lambda_{m}^{*}\right)$. 
FATO 3.15.1: $B$ é viável em $P_{i+1}$.

\section{DEMONSTRAÇÃO:}

$A$ é viável em $P i$, portanto

$$
\begin{aligned}
& \sum_{\substack{j \neq i \\
j=1}}^{m} \lambda_{j}^{*}=\lambda \\
& 0 \leq \lambda_{j}^{*}<\mu_{j} \quad j \in\{1,2, \ldots,(i-1),(i+1), \ldots, m\}
\end{aligned}
$$

Para mostrar que $B$ é viável em $P_{i+1}$, resta mostrar que $\lambda_{i+1}^{*}<\mu_{i}$.

Mas $\lambda_{i+1}^{*}<\mu_{i+1} \leq \mu_{i}$ e portanto $B$ é viável em $P_{i+1}$.

FATO 3.15.2: $T(B) \leq T(A) \quad$ se e somente se $\quad \mu_{i} \geq \mu_{i+1}$. Sendo que

$T(B)<T(A) \quad$ se e somente se $\quad \mu_{i}>\mu_{i+1}$

$T(B)=T(A) \quad$ se e somente se $\quad \mu_{i}=\mu_{i+1}$

\section{DEMONSTRAÇÃO:}

$T(B)=\frac{1}{\lambda}\left(\sum_{j=1}^{i-1} \frac{\lambda_{j}^{*}}{\mu_{j}-\lambda_{j}^{*}}+\frac{\lambda_{i+1}^{*}}{\mu_{i}-\lambda_{i+1}^{*}}+0+\sum_{j=i+2}^{m} \frac{\lambda_{j}^{*}}{\mu_{j}-\lambda_{j}^{*}}\right)$

$T(A)=\frac{1}{\lambda}\left(\sum_{j=1}^{i-1} \frac{\lambda_{j}^{*}}{\mu_{j}-\lambda_{j}^{*}}+0+\frac{\lambda_{i+1}^{*}}{\mu_{i+1}-\lambda_{i+1}^{*}}+\sum_{j=i+2}^{m} \frac{\lambda_{j}^{*}}{\mu_{j}-\lambda_{j}^{*}}\right)$

Logo

$$
\begin{aligned}
& T(B) \leq T(A) \\
\Longleftrightarrow & \frac{\lambda_{i+1}^{*}}{\mu_{i}-\lambda_{i+1}^{*}} \leq \frac{\lambda_{i+1}^{*}}{\mu_{i+1}-\lambda_{i+1}^{*}} \\
\Longleftrightarrow & \mu_{i} \geq \mu_{i+1}
\end{aligned}
$$

É fácil notar que

$T(B)<T(A)$ se e somente se $\mu_{i}>\mu_{i+1} \quad$ e

$T(B)=T(A)$ se e somente se $\mu_{i}=\mu_{i+1}$.

Temos então que se $\mu_{i} \geq \mu_{i+1}$

$$
\begin{aligned}
& \text { valor ótimo }\left(P_{i}\right)=T(A) \\
& \text { (por (1)) } \\
& \geq T(B) \\
& \text { (pelo Fato 3.15.2) } \\
& \geq \text { valor ótimo }\left(P_{i+1}\right)
\end{aligned}
$$

Se $\mu_{i}>\mu_{i+1}$, pelo Fato 3.15 .2 , temos 
$T(A)>T(B)$ e portanto valor ótimo $(P i)>$ valor ótimo $\left(P_{i+1}\right)$.

Analogamente, se $\mu_{i}=\mu_{i+1}$ temos que valor ótimo $\left(P_{i}\right)=$ valor ótimo $\left(P_{i+1}\right)$.

COROLÁRIO 3.16: Se $\mu_{1} \geq \mu_{2} \geq \ldots \mu_{m}$ temos

valor ótimo $\left(P_{m}\right) \leq$ valor ótimo $\left(P_{m-1}\right) \leq \ldots \leq$ valor ótimo $\left(P_{1}\right)$.

\section{DEMONSTRAÇÃO:}

Imediata a partir do teorema 3.15 .

Do corolário 3.16 surge naturalmente a seqüencia de problemas $P L_{p}, \quad p=m,(m-$ 1), . , 2, onde cada $P l_{p}$ corresponde ao problem $P_{o}$ considerando $p$ componentes 'ativas' para o conjunto de pontos viáveis, ou seja, um ponto viável $\left(x_{1}, x_{2}, \ldots, x_{p}, x_{p+1}, \ldots, x_{m}\right)$ é tal que $x_{p+1}=\ldots=x_{m}=0$. Assim cada $P L_{p}$ é definido por:

$$
\begin{aligned}
P L_{p}: \quad \min & T\left(x_{1}, x_{2}, \ldots, x_{p}, 0, \ldots, 0\right) \\
\text { sujeito a } & \sum_{i=1}^{p} x_{i}=\lambda \\
& 0 \leq x_{i} \leq \mu_{i} \quad i=1,2, \ldots, p
\end{aligned}
$$

Esta definição decorre do fato que pelo corolário 3.16, quando se quer zerar uma componente o melhor é zerar a última $\left(x_{m}=0\right)$, quando se quer zerar duas componentes o melhor é zerar as duas últimas $\left(x_{m-1}=x_{m}=0\right)$ e assim por diante. Obviamente isto pressupõe $\mu_{1} \geq \mu_{2} \geq \ldots \geq \mu_{m}$, que é a hipótese básica do corolário 3.16 .

TEOREMA 3.17: Consideremos as condições de Lagrange para a seqüência de problemas $P L_{p}, \quad p=m,(m-1), \ldots, 2$ acima definidos. Se $\mu_{1} \geq \mu_{2} \geq \ldots \geq \mu_{m}$, vale que:

a) Para o ponto $\left(\ell_{1}^{*}, \ldots, \ell_{p}^{*}, 0 \ldots, 0\right)$ que obedece Lagrange para $P L_{p}$, $\exists \ell_{p-1}, \quad 0 \leq \ell_{p-1}<\mu, \quad$ tal que $\quad \ell_{j}^{*}>0 \quad j=1,2, \ldots, p$ para $\lambda>\ell_{p-1}$, e é dado por

$$
\ell_{p-1}=\sum_{j=1}^{p-1} \mu_{j}-\sqrt{\mu_{p}} \sum_{j=1}^{p-1} \sqrt{\mu_{j}}
$$

b) $\ell_{i} \geq \ell_{i-1} \quad \forall i \in\{(m-1), \ldots, 2\}$ sendo que

$\ell_{i}=\ell_{i-1} \quad$ se e somente se $\quad \mu_{i}=\mu_{i+1}$

$\ell_{i}>\ell_{i-1} \quad$ se e somente se $\quad \mu_{i}>\mu_{i+1}$ 
Observação: Não é necessário considerar o caso $p=1$ pois basta tomar $\ell_{0}=0$ e $\lambda>\ell_{0}$ para ter pelo menos uma componente ativa. É interessante observar que $\ell_{1}>0$.

\section{DEMONSTRAÇÃO:}

a) $\left(\ell_{1}^{*}, \ldots, \ell_{p}^{*}, 0, \ldots, 0\right)$ obedece Lagrange para $P L_{p}$, portanto

$$
\begin{gathered}
\ell_{i}^{*}=\frac{\sqrt{\mu_{i}}}{\sum_{j=1}^{p} \sqrt{\mu_{j}}} * \lambda+\mu_{i}-\sum_{j=1}^{p} \mu_{j} \frac{\sqrt{\mu_{i}}}{\sum_{j=1}^{p} \sqrt{\mu_{j}}} \quad i=1,2, \ldots, p \\
\ell_{i}^{*}<0 \\
\Longleftrightarrow \sqrt{\mu_{i}}+\lambda+\mu_{i} \sum_{j=1}^{p} \sqrt{\mu_{j}}-\sum_{j=1}^{p} \mu_{j} \sqrt{\mu_{i}}<0 \\
\Longleftrightarrow \lambda<\sum_{j=1}^{p} \mu_{j}-\sqrt{\mu_{i}} \sum_{j=1}^{p} \sqrt{\mu_{j}}
\end{gathered}
$$

Seja $\bar{\ell}_{i}^{*}=\sum_{j=1}^{p} \mu_{j}-\sqrt{\mu_{i}} \sum_{j=1}^{p} \sqrt{\mu_{j}} \quad i=1,2, \ldots, p$

Temos que $\bar{\ell}_{1}^{*} \leq \bar{\ell}_{2}^{*} \leq \ldots \leq \bar{\ell}_{p}^{*}$

pois se tomarmos $u, v \in\{1,2, \ldots\}, \mu<v$

$$
\bar{\ell}_{u}^{*}=\sum_{j=1}^{p} \mu_{j}-\sqrt{\mu_{u}} \sum_{j=1}^{p} \sqrt{\mu_{j}} \leq \sum_{j=1}^{p} \mu_{j}-\sqrt{\mu_{v}} \sum_{j=1}^{p} \sqrt{\mu_{j}}=\bar{\ell}_{v}^{*}
$$

Definamos então $\ell_{p-1}=\bar{\ell}_{p}^{*}$

$$
\ell_{p-1}=\sum_{j=1}^{p} \mu_{j}-\sqrt{\mu_{p}} \sum_{j=1}^{p} \sqrt{\mu_{j}}=\sum_{j=1}^{p-1} \mu_{j}-\sqrt{\mu_{p}} \sum_{j=1}^{p-1} \sqrt{\mu_{j}}
$$

Por (1) e (2) temos que $\ell_{i}^{*}>0 \quad i=1,2, \ldots, p \quad$ para $\lambda<\ell_{p-1}$.

Resta mostrar que $0 \leq \ell_{p-1}<\mu$.

Mas

$$
\ell_{p-1}=\sum_{j=1}^{p-1} \mu_{j}-\sqrt{\mu_{p}} \sum_{j=1}^{p-1} \sqrt{\mu_{j}} \geq \sum_{j=1}^{p-1} \mu_{j}-\sum_{j=1}^{p-1} \sqrt{\mu_{j}} \sqrt{\mu_{j}}=0
$$

É interessante notar que $\ell_{p-1}=0$ se e somente se $\mu_{1}=\mu_{2}=\ldots=\mu_{p-1}$.

E também

$$
\ell_{p-1}=\sum_{j=1}^{p-1} \mu_{j}-\sqrt{\mu_{p}} \sum_{j=1}^{p-1} \sqrt{\mu_{j}}<\sum_{j=1}^{p-1} \mu_{j}<\mu
$$


b) $\ell_{i}-\ell_{i-1}=\sum_{j=1}^{i} \mu_{j}-\sqrt{\mu_{i+1}} \sum_{j=1}^{i} \sqrt{\mu_{j}}-\left(\sum_{j=1}^{i-1} \mu_{j}-\sqrt{\mu_{i}} \sum_{j=1}^{i-1} \sqrt{\mu_{j}}\right)$

$$
\begin{aligned}
& =\mu_{i}+\sum_{j=1}^{i-1} \sqrt{\mu_{j}}\left(\sqrt{\mu_{i}}-\sqrt{\mu_{i+1}}\right)-\sqrt{\mu_{i+1}} \sqrt{\mu_{i}} \\
& =\sqrt{\mu_{i}}\left(\sqrt{\mu_{i}}-\sqrt{\mu_{i+1}}\right)+\sum_{j=1}^{i-1} \sqrt{\mu_{j}}\left(\sqrt{\mu_{i}}-\sqrt{\mu_{i+1}}\right) \\
& =\left(\sqrt{\mu_{i}}-\sqrt{\mu_{i+1}}\right) \sum_{j=1}^{i} \sqrt{\mu_{j}} \geq 0
\end{aligned}
$$

e ainda

$\ell_{i}=\ell_{i-1} \quad$ se e somente se $\quad \sqrt{\mu_{i}}=\sqrt{\mu_{i+1}}$

$\ell_{i}>\ell_{i-1} \quad$ se e somente se $\sqrt{\mu_{i}}>\sqrt{\mu_{i+1}}$

A distribuição ótima da carga entre as origens é dada pela solução do $\lambda$-problema.

Obviamente para $\lambda<0$ e $\lambda \geq \mu$ não existe solução para o $\lambda$-problema e o caso $\lambda=0$ não tem interesse prático.

Enunciaremos o teorema 3.18, que descreve a solução do $\lambda$-problema para $0<\lambda<\mu$. Denotaremos esta solução por $\left(\lambda_{1}^{*}, \lambda_{2}^{*}, \ldots, \lambda_{m}^{*}\right)$.

TEOREMA 3.18: Para $\mu \geq \mu_{2} \ldots \geq \mu_{m}$, e $0<\lambda<\mu$, a solução do $\lambda$-problema obedece à seguinte propriedade:

$\exists \ell_{1}, \ell_{2}, \ldots, \ell_{m-1} \quad$ ordenados crescente $\quad\left(\ell_{1} \leq \ell_{2} \leq \ldots \leq \ell_{m-1}\right)$

tais que

$$
\begin{aligned}
& \left.\lambda \in] \ell_{i-1}, \ell_{i}\right] \Rightarrow\left[\lambda_{j}^{*}>0 \Longleftrightarrow j \leq i\right] \quad \text { para } i=1,2, \ldots,(m-1) \\
& \lambda \in] \ell_{m-1}, \mu\left[\Rightarrow\left[\lambda_{j}^{*}>0 \Longleftrightarrow j \leq m\right]\right.
\end{aligned}
$$

onde $\ell_{0}=0 \quad \mathrm{e}$

$$
\lambda_{j}^{*}=\frac{\sqrt{\mu_{j}}}{\sum_{i=1}^{j} \sqrt{\mu_{i}}} * \lambda+\mu_{j}-\sum_{i=1}^{j} \mu_{i} \frac{\sqrt{\mu_{j}}}{\sum_{i=1}^{j} \sqrt{\mu_{i}}}
$$

\section{DEMONSTRAÇÃO:}

Vamos demonstrar por indução em $m$. $m=2$

Para $\mu_{1}>\mu_{2}$, pelo teorema 3.7 , basta tomar $\ell_{1}=\bar{\lambda}$, segue que

$$
\begin{array}{cccc}
\lambda \leq \ell_{1} & \Rightarrow & \lambda_{1}^{*}=\lambda & \lambda_{2}^{*}=0 \\
\lambda \in] \ell_{1}, \mu[ & \Rightarrow & \lambda_{1}^{*}>0 & \lambda_{2}^{*}>0
\end{array}
$$

Para $\mu_{1}=\mu_{2}$, pelo teorema 3.9, temos que a solução de $P_{o}$ é tal que $\lambda_{1}^{*}=\lambda_{2}^{*}=\frac{\lambda}{2}>0, \quad \forall 0<\lambda<\mu, \quad$ bastando então tomar $\ell_{1}=0$. 
Hipótese de Indução

Para $(m-1)$ servidores com capacidades $\mu_{1}, \ldots, \mu_{m-1}, \quad \mu_{1} \geq \mu_{2} \geq \ldots \geq \mu_{m-1}$ $\exists \ell_{1}, \ell_{2}, \ldots, \ell_{m-2} \quad$ ordenados crescente e $\ell_{0}=0$ tais que

$$
\begin{aligned}
& \left.\lambda \in] \ell_{i-1}, \ell_{i}\right] \Rightarrow\left[\lambda_{j}^{*}>0 \Longleftrightarrow j \leq i\right] \quad i=1,2, \ldots,(m-2) \\
& \lambda>\ell_{m-2} \Rightarrow\left[\lambda_{j}^{*}>0 \Longleftrightarrow j \leq m-1\right]
\end{aligned}
$$

\section{Passo de Indução}

Sejam $\left(\ell_{1}^{*}, \ell_{2}^{*}, \ldots, \ell_{m}^{*}\right)$ na forma do teorema 3.12 e $\ell_{m-1}$ conforme o teorema 3.17 .

Para $\lambda>\ell_{m-1}$, temos então que $\left(\ell_{1}^{*}, \ell_{2}^{*}, \ldots, \ell_{m}^{*}\right)$ é viável para $P_{x}$, e pelo teorema 3.14 , temos que $\left(\ell_{1}^{*}, \ell_{2}^{*}, \ldots, \ell_{m}^{*}\right)$ solve $P_{x}$.

FATO 3.18.1: Para $\lambda>\ell_{m-1}$, a solução ótima de $P_{x}$ solve $P_{o}$.

DEMONSTRAÇÃO:

Vamos demonstrar por indução em $m$

$m=2$

Já foi mostrado no teorema 3.7.

\section{Hipótese de Indução}

Para $\lambda>\ell_{m-2}$, a solução do problema com $(m-1)$ servidores é dada pela solução de Lagrange, ou seja, pela solução com $(m-1)$ componentes 'ativas'.

\section{Passo de Indução}

Tomemos $\lambda>\ell_{m-1}$. Pelo teorema 3.17 temos $\lambda>\ell_{m-2}$.

A solução ótima de $P_{o}$ é dada pela solução do problema $P_{x}$ ou pela solução de $P_{m}$ (de acordo com os teoremas 3.10 e 3.11 e corolário 3.16$)$.

Pela Hipótese de Indução temos que a solução de $P_{m}$ é dada por $A=\left(a_{1}^{*}, a_{2}^{*}, \ldots, a_{m-1}^{*}, 0\right) \quad \operatorname{com} a_{i}^{*}>0, i=1, \ldots,(m-1)$.

Seja $B=\left(a_{1}^{*}-\Delta, a_{2}^{*}, \ldots, a_{m-1}^{*}, \Delta\right), \quad 0<\Delta<\min \left(a_{1}^{*}, \mu_{m}\right)$.

É fácil verificar que $T(B)<T(A) \quad$ para $\lambda>\ell_{m-1}$ 


$$
\begin{aligned}
& T(B)=\frac{1}{\lambda}\left(\frac{a_{1}^{*}-\Delta}{\mu_{1}-a_{1}^{*}+\Delta}+\sum_{i=2}^{m-1} \frac{a_{i}^{*}}{\mu_{i}-a_{i}^{*}}+\frac{\Delta}{\mu_{m}-\Delta}\right) \\
& T(A)=\frac{1}{\lambda} \sum_{i=1}^{m-1} \frac{a_{i}^{*}}{\mu_{i}-a_{i}^{*}} \\
& T(B)<T(A) \\
\Longleftrightarrow & \frac{a_{1}^{*}-\Delta}{\mu_{1}-a_{1}^{*}+\Delta}+\frac{\Delta}{\mu_{m}-\Delta}<\frac{a_{1}^{*}}{\mu_{1}-a_{1}^{*}} \\
\Longleftrightarrow & \left(a_{1}^{*}-\Delta\right)\left(\mu_{m}-\Delta\right)\left(\mu_{1}-a_{1}^{*}\right)+\Delta\left(\mu_{1}-a_{1}^{*}+\Delta\right)\left(\mu_{1}-a_{1}^{*}\right)<a_{1}^{*}\left(\mu_{1}-a_{1}^{*}+\Delta\right)\left(\mu_{m}-\Delta\right) \\
\Longleftrightarrow & -\Delta\left(\mu_{m}-\Delta\right)\left(\mu_{1}-a_{1}^{*}\right)+\Delta\left(\mu_{1}-a_{1}^{*}+\Delta\right)\left(\mu_{1}-a_{1}^{*}\right)<a_{1}^{*} \Delta\left(\mu_{m}-\Delta\right) \\
\Longleftrightarrow & -\left(\mu_{m}-\Delta\right) \mu_{1}+\left(\mu_{1}-a_{1}^{*}+\Delta\right)\left(\mu_{1}-a_{1}^{*}\right)<0 \\
\Longleftrightarrow & \Delta\left(\mu_{1}+\mu_{1}-a_{1}^{*}\right)<\mu_{1} \mu_{m}-\left(\mu_{1}-a_{1}^{*}\right)^{2} \\
\Longleftrightarrow & \Delta<\frac{\mu_{1} \mu_{m}-\left(\mu_{1}-a_{1}^{*}\right)^{2}}{2 \mu_{1}-a_{1}^{*}}
\end{aligned}
$$

Seja $X=\sum_{j=1}^{m-1} \mu_{j} \quad$ e $\quad Y=\sum_{j=1}^{m-1} \sqrt{\mu_{j}}$

Temos que $\lambda>\ell_{m-1}=X-\sqrt{\mu_{m}} Y \quad$ e $\quad a_{1}^{*}=\mu_{1}-\sqrt{\mu_{1}} \frac{(X-\lambda)}{Y}$

Assim

$$
\begin{aligned}
\mu_{1}-a_{1}^{*} & =\sqrt{\mu_{1}} \frac{(X-X)}{Y}=\sqrt{\mu_{1}} \frac{X}{Y}-\lambda \frac{\sqrt{\mu_{1}}}{Y} \\
& <\sqrt{\mu_{1}} \frac{X}{Y}-\left(X-\sqrt{\mu_{m}} Y\right) \frac{\sqrt{\mu_{1}}}{Y}=\sqrt{\mu_{1}} \sqrt{\mu_{m}}
\end{aligned}
$$

Portanto $\left(\mu_{1}-a_{1}^{*}\right)^{2}<\mu_{1} \mu_{m}$.

Como $a_{1}^{*}<\mu_{1}<2 \mu_{1}$, temos que

$$
\frac{\mu_{1} \mu_{2}-\left(\mu_{1}-a_{1}^{*}\right)^{2}}{2 \mu_{1}-a_{1}^{*}}>0 .
$$

Assim, $\exists \Delta>0$ tal que $T(B)<T(A)=$ valor ótimo $\left(P_{m}\right)$ e como $B$ é viável em $P_{x}$, temos que a solução ótima de $P_{o}$ é dada pela solução de $P_{x}$.

Logo, para $\lambda<\ell_{m-1}$ a solução ótima de $P_{x}$ solve $P_{o}$, bastando então tomar $\lambda_{i}^{*}=\ell_{i}^{*}>0 \quad i=1,2, \ldots, m$.

Passemos para a análise do caso $\lambda \leq \ell_{m-1}$. 
Vamos mostrar que para $\lambda \leq \ell_{m-1}$, a solução ótima é tal que a carga no $m$-ésimo servidor $\left(\lambda_{m}^{*}\right)$ é igual a zero.

Para tanto, sejam

$p \lambda$ a carga no m-ésimo servidor, $p \in[0,1]$

$\lambda^{\prime}=(1-p) \lambda$

a carga nos demais servidores (servidores $1,2, \ldots,(m-1))$

$\mu^{\prime}=\sum_{i=1}^{m-1} \mu_{i}$

Considerando o subsistema $S\left(\lambda^{\prime}, \mu^{\prime}, m-1, m-1\right)$, constituído dos $(m-1)$ primeiros grupos do sistema original, seja $\left(x_{1}^{*}, x_{2}^{*}, \ldots, x_{m-1}^{*}\right)$ dado conforme o teorema 3.12 por

$$
x_{i}^{*}=\mu_{i}-\left(\mu^{\prime}-\lambda^{\prime}\right) \frac{\sqrt{\mu_{i}}}{\sum_{j=1}^{m-1} \sqrt{\mu_{j}}} \quad i=1,2, \ldots,(m-1)
$$

E então

$$
\begin{aligned}
\frac{x_{i}^{*}}{\mu_{i}-x_{i}^{*}} & =\frac{\mu_{i}-\left(\mu^{\prime}-\lambda^{\prime}\right) \frac{\sqrt{\mu_{i}}}{\sum_{j=1}^{m-1} \sqrt{\mu_{j}}}}{\left(\mu^{\prime}-\lambda^{\prime}\right) \frac{\sqrt{\mu_{i}}}{\sum_{j=1}^{m-1} \sqrt{\mu_{j}}}}=\frac{\mu_{i} \sum_{j=1}^{m-1} \sqrt{\mu_{j}}-\left(\mu^{\prime}-\lambda^{\prime}\right) \sqrt{\mu_{i}}}{\left(\mu^{\prime}-\lambda^{\prime}\right) \sqrt{\mu_{i}}} \\
& =\frac{\sqrt{\mu_{i}} \sum_{j=1}^{m-1} \sqrt{\mu_{j}}}{\mu^{\prime}-\lambda^{\prime}}-1 \quad i=1,2, \ldots,(m-1)
\end{aligned}
$$

Seja a função $\theta$ definida por

$$
\theta(p)=\frac{p \lambda}{\mu_{m}-p \lambda}+\sum_{i=1}^{m-1}\left(\frac{\sqrt{\mu_{i}} \sum_{j=1}^{m-1} \sqrt{\mu_{j}}}{\sum_{j=1}^{m-1} \mu_{j}-(1-p) \lambda}-1\right)
$$

Temos que $\left.\left.\lambda_{m}^{*}=0 \Longleftrightarrow(\theta(p)>\theta(0), \quad \forall p \in] 0,1\right]\right)$

$$
\begin{aligned}
& \theta(p)>\theta(0) \quad \forall p \in] 0,1] \\
\Longleftrightarrow & \frac{p \lambda}{\mu_{m}-p \lambda}+\sum_{i=1}^{m-1}\left(\frac{\sqrt{\mu_{i}} \sum_{j=1}^{m-1} \sqrt{\mu_{j}}}{\sum_{j=1}^{m-1} \mu_{j}-(1-p) \lambda}-1\right)>\sum_{i=1}^{m-1}\left(\frac{\sqrt{\mu_{i}} \sum_{j=1}^{m-1} \sqrt{\mu_{j}}}{\sum_{j=1}^{m-1} \mu_{j}-\lambda}-1\right)
\end{aligned}
$$

Seja $A=\sum_{j=1}^{m-1} \mu_{j}-\lambda$.

Como $\lambda \leq \ell_{m-1}=\sum_{j=1}^{m-1} \mu_{j}-\sqrt{\mu_{m}} \sum_{j=1}^{m-1} \sqrt{\mu_{j}}<\sum_{j=1}^{m-1} \mu_{j}$, temos $A>0$. 
Temos então que

$$
\begin{aligned}
& \lambda_{m}^{*}=0 \\
\Longleftrightarrow & p \lambda(A+p \lambda) A+\left(\mu_{m}-p \lambda\right) A \sum_{i=1}^{m-1} \sqrt{\mu_{i}} \sum_{j=1}^{m-1} \sqrt{\mu_{j}} \\
& >\left(\mu_{m}-p \lambda\right)(A+p \lambda) \sum_{i=1}^{m-1} \sqrt{\mu_{i}} \sum_{j=1}^{m-1} \sqrt{\mu_{j}} \\
\Longleftrightarrow & \left.p \lambda(A+p \lambda) A+\left(\mu_{m}-p \lambda\right)(-p \lambda) \sum_{i=1}^{m-1} \sqrt{\mu_{i}} \sum_{j=1}^{m-1}\right) \sqrt{\mu_{j}}>0 \\
\Longleftrightarrow & (A+p \lambda) A-\left(\mu_{m}-p \lambda\right) \sum_{i=1}^{m-1} \sqrt{\mu_{i}} \sum_{j=1}^{m-1} \sqrt{\mu_{j}}>0
\end{aligned}
$$

Seja $F(p)=(A+p \lambda) A-\left(\mu_{m}-p \lambda\right) \sum_{i=1}^{m-1} \sqrt{\mu_{i}} \sum_{j=1}^{m-1} \sqrt{\mu_{j}}$

$$
=A^{2}+A p \lambda-\left(\mu_{m}-p \lambda\right)\left(\sum_{i=1}^{m-1} \sqrt{\mu_{i}}\right)^{2}
$$

Temos então que $\left.\left.\quad \lambda_{m}^{*}=0 \Longleftrightarrow(F(p)>0, \quad \forall p \in] 0,1\right]\right)$.

i) $F(0)=A^{2}-\mu_{m}\left(\sum_{i=1}^{m-1} \sqrt{\mu_{i}}\right)^{2}$

$$
\begin{aligned}
& A^{2}-\mu_{m}\left(\sum_{i=1}^{m-1} \sqrt{\mu_{i}}\right)^{2} \geq 0 \\
\Longleftrightarrow & A-\sqrt{\mu_{m}} \sum_{i=1}^{m-1} \sqrt{\mu_{i}} \geq 0 \quad \text { e } \quad A+\sqrt{\mu_{m}} \sum_{i=1}^{m-1} \sqrt{\mu_{i}} \geq 0 \\
\Longleftrightarrow & \sum_{i=1}^{m-1} \mu_{i}-\lambda-\sqrt{\mu_{m}} \sum_{i=1}^{m-1} \sqrt{\mu_{i}} \geq 0 \quad \text { e } \sum_{i=1}^{m-1} \mu_{i}-\lambda+\sqrt{\mu_{m}} \sum_{i=1}^{m-1} \sqrt{\mu_{i}} \geq 0 \\
\Longleftrightarrow & \lambda \leq \sum_{i=1}^{m-1} \mu_{i}-\sqrt{\mu_{m}} \sum_{i=1}^{m-1} \sqrt{\mu_{i}} \quad \text { e } \quad \lambda \leq \sum_{i=1}^{m-1} \mu_{i}+\sqrt{\mu_{m}} \sum_{i=1}^{m-1} \sqrt{\mu_{i}} \\
\Longleftrightarrow & \lambda \leq \sum_{i=1}^{m-1} \mu_{i}-\sqrt{\mu_{m}} \sum_{i=1}^{m-1} \sqrt{\mu_{i}}=\ell_{m-1}
\end{aligned}
$$

Portanto temos $F(0)>0, \quad \forall \lambda<\ell_{m-1} \quad$ e $\quad F(0)=0, \quad \lambda=\ell_{m-1}$

ii) $F(1)=A^{2}+A \lambda-\left(\mu_{m}-\lambda\right)\left(\sum_{i=1}^{m-1} \sqrt{\mu_{i}}\right)^{2}=G(\lambda)$

$$
\begin{aligned}
G(\lambda) & =\left(\sum_{j=1}^{m-1} \mu_{j}-\lambda\right)^{2}+\left(\sum_{j=1}^{m-1} \mu_{j}-\lambda\right) \lambda-\left(\mu_{m}-\lambda\right)\left(\sum_{j=1}^{m-1} \sqrt{\mu_{j}}\right)^{2} \\
& =\left(\sum_{j=1}^{m-1} \mu_{j}\right)^{2}-2 \lambda \sum_{j=1}^{m-1} \mu_{j}+\lambda^{2}+\lambda \sum_{j=1}^{m-1} \mu_{j}-\lambda^{2}-\mu_{m}\left(\sum_{j=1}^{m-1} \sqrt{\mu_{j}}\right)^{2}+\lambda\left(\sum_{j=1}^{m-1} \sqrt{\mu_{j}}\right)^{2} \\
& =\left(\sum_{j=1}^{m-1} \mu_{j}\right)^{2}-\lambda \sum_{j=1}^{m-1} \mu_{j}+\left(\lambda-\mu_{m}\right)\left(\sum_{j=1}^{m-1} \sqrt{\mu_{j}}\right)^{2}
\end{aligned}
$$


Capítulo 3: ANÁLISE DO ALGORITMO PARTIÇÃO DOS SERVIDORES

$$
\begin{aligned}
= & \sum_{j=1}^{m-1} \mu_{j}\left(\sum_{k=1}^{m-1} \mu_{k}-\lambda\right)+\left(\lambda-\mu_{m}\right)\left(\sum_{j=1}^{m-1} \mu_{j}+\sum_{i=1}^{m-1} \sum_{\substack{j \neq i \\
j=1}}^{m-1} \sqrt{\mu_{i}} \sqrt{\mu_{j}}\right) \\
= & \sum_{j=1}^{m-1} \mu_{j}\left(\sum_{j=1}^{m-1} \mu_{k}-\mu_{m}\right)+\left(\lambda-\mu_{m}\right) \sum_{i=1}^{m-1} \sum_{j \neq i}^{m-1} \sqrt{\mu_{i}} \sqrt{\mu_{j}} \\
= & \sum_{j=1}^{m-1} \mu_{j}\left(\sum_{k=1}^{m-2} \mu_{k}+\mu_{m-1}-\mu_{m}\right)+2\left(\lambda-\mu_{m}\right) \sum_{i=1}^{m-1} \sum_{j=i+1}^{m-1} \sqrt{\mu_{i}} \sqrt{\mu_{j}} \\
> & \sum_{j=1}^{m-1} \mu_{j}\left(\sum_{k=1}^{m-2} \mu_{k}\right)-2 \mu_{m} \sum_{i=1}^{m-1} \sum_{j=i+1}^{m-1} \sqrt{\mu_{i}} \sqrt{\mu_{j}} \\
= & \sum_{j=1}^{m-2} \mu_{j}^{2}+2 \sum_{i=1}^{m-2} \sum_{j=i+1}^{m-2} \mu_{i} \mu_{j}+\mu_{m-1} \sum_{j=1}^{m-2} \mu_{j}-2 \mu_{m} \sum_{i=1}^{m-2} \sum_{j=i+1}^{m-1} \sqrt{\mu_{i}} \sqrt{\mu_{j}} \\
= & \sum_{j=1}^{m-2} \mu_{j}^{2}+\mu_{m-1} \sum_{j=1}^{m-2} \mu_{j}-2 \mu_{m} \sqrt{\mu_{m-1}} \sum_{j=1}^{m-2} \sqrt{\mu_{j}} \\
& +2 \sum_{i=1}^{m-2} \sum_{j=i+1}^{m-2}\left(\mu_{i} \mu_{j}-\mu_{m} \sqrt{\mu_{i}} \sqrt{\mu_{j}}\right) \\
= & \sum_{j=1}^{m-2}\left(\mu_{j}^{2}+\mu_{m-1} \mu_{j}-2 \mu_{m} \sqrt{\mu_{m-1}} \sqrt{\mu_{j}}\right)+2 \sum_{i=1}^{m-2} \sum_{j=i+1}^{m-2}\left(\mu_{i} \mu_{j}-\mu_{m} \sqrt{\mu_{i}} \sqrt{\mu_{j}}\right)
\end{aligned}
$$

Seja $X=\mu_{j}^{2}+\mu_{m-1} \mu_{j}-2 \mu_{m} \sqrt{\mu_{m-1}} \sqrt{\mu_{j}}$

$$
=\sqrt{\mu_{m-1}} \sqrt{\mu_{j}}\left(\sqrt{\mu_{j}} \frac{\mu_{j}}{\sqrt{\mu_{m-1}}}+\sqrt{\mu_{m-1}} \sqrt{\mu_{j}}-2 \mu_{m}\right)
$$

Como $\sqrt{\mu_{j}} \frac{\mu_{j}}{\sqrt{\mu_{m-1}}} \geq \mu_{j} \geq \mu_{m} \quad$ e $\quad \sqrt{\mu_{m-1}} \sqrt{\mu_{j}} \geq \mu_{j} \geq \mu_{m}$

temos que $X \geq\left(\mu_{m}+\mu_{m}-2 \mu_{m}\right)=0$.

Seja $Y=\mu_{i} \mu_{j}-\mu_{m} \sqrt{\mu_{i}} \sqrt{\mu_{j}}=\sqrt{\mu_{i}} \sqrt{\mu_{j}}\left(\sqrt{\mu_{i}} \sqrt{\mu_{j}}-\mu_{m}\right)$

Como $\sqrt{\mu_{i}} \geq \sqrt{\mu_{m}} \quad$ e $\quad \sqrt{\mu_{j}} \geq \sqrt{\mu_{m}}$

temos que $\sqrt{\mu_{i}} \sqrt{\mu_{j}}-\mu_{m} \geq \sqrt{\mu_{m}} \sqrt{\mu_{m}}-\mu_{m}=0$ e portanto $\quad Y \geq 0$

Por (A), (B) e (C) temos $G(Y)>0$ e portanto $F(1)>0$.

iii) $F(p)=p F(1)+(1-p) F(0) \quad \forall p \in[0,1]$

Por i) temos $F(0) \geq 0, \quad \forall \lambda \leq \ell_{m-1}$

Por ii) temos $F(1)>0$

Logo temos

$F(p)>0 \quad \forall p \in] 0,1], \quad \forall \lambda \leq \ell_{m-1}$ 
E portanto, temos $\lambda_{m}^{*}=0$ para $\lambda \leq \ell_{m-1}$

Restam então $(m-1)$ servidores e recaímos na Hipótese de Indução, logo $\exists \ell_{1}, \ldots, \ell_{m-2}$ ordenados crescente e $\ell_{0}=0$ tais que

$$
\begin{aligned}
\left.\lambda \in] \ell_{i-1}, \ell_{i}\right] & \Rightarrow\left[\lambda_{j}^{*}>0 \Longleftrightarrow j \leq i\right] \quad i=1,2, \ldots,(m-2) \\
\lambda>\ell_{m-2} & \Rightarrow\left[\lambda_{j}^{*}>0 \Longleftrightarrow j \leq m-1\right]
\end{aligned}
$$

Pelo Teorema 3.17 (b) temos que $\ell_{m-2} \leq \ell_{m-1}$ e por (1) temos que

$$
\left.\lambda \in] \ell_{m-2}, \ell_{m-1}\right] \Rightarrow\left[\lambda_{j}^{*}>0 \Longleftrightarrow j \leq m-1\right]
$$

Pelo Fato 3.18.1, temos que

$$
\lambda \in] \ell_{m-1}, \mu\left[\Rightarrow\left[\lambda_{j}^{*}>0 \Longleftrightarrow j \leq m\right]\right.
$$

Pelo método de demonstração e de construção dos $\ell_{i}^{\prime} s$ temos que

$$
\lambda_{j}^{*}=\frac{\sqrt{\mu_{j}}}{\sum_{i=1}^{j} \sqrt{\mu_{i}}} * \lambda+\mu_{j}-\sum_{i=1}^{j} \sqrt{\mu_{i}} \frac{\sqrt{\mu_{j}}}{\sum_{i=1}^{j} \sqrt{\mu_{i}}}
$$

COROLÁRIO 3.19: Se $\mu_{1}>\mu_{2}>\ldots>\mu_{p}=\mu_{p+1}>\mu_{p+2}>\ldots>\mu_{m} \quad \operatorname{com} p<m-1$ temos

$$
\begin{aligned}
& \left.\lambda \in] \ell_{i-1}, \ell_{i}\right] \quad \Rightarrow\left[\lambda_{j}^{*}>0 \Longleftrightarrow j \geq i\right] \quad i=1,2, \ldots,(p-1) \\
& \left.\lambda \in] \ell_{p-1}, \ell_{p+1}\right] \Rightarrow\left[\lambda_{j}^{*}>0 \Longleftrightarrow j \leq p+1\right] \\
& \left.\lambda \in] \ell_{i-1}, \ell_{i}\right] \quad \Rightarrow\left[\lambda_{j}^{*}>0 \Longleftrightarrow j \leq i\right] \quad i=(p+2), \ldots,(m-1) \\
& \lambda \in] \ell_{m-1}, \mu\left[\quad \Rightarrow\left[\lambda_{j}^{*}>0 \Longleftrightarrow j \leq m\right]\right.
\end{aligned}
$$

e ainda $\lambda_{p}^{*}=\lambda_{p+1}^{*}$

\section{DEMONSTRAÇÃO:}

Imediata, observando-se que pelo teorema $3.17 . \mathrm{b}, \ell_{p-1}=\ell_{p}$.

COROLÁRIO 3.20: Se $\mu_{1}>\mu_{2}>\ldots>\mu_{m-1}=\mu_{m}$ temos

$$
\begin{aligned}
& \left.\lambda \in] \ell_{i-1}, \ell_{i}\right] \Rightarrow\left[\lambda_{j}^{*}>0 \Longleftrightarrow j \leq i\right] \quad i=1,2, \ldots,(m-2) \\
& \lambda \in] \ell_{m-2}, \mu\left[\Rightarrow\left[\lambda_{j}^{*}>0 \Longleftrightarrow j \leq m\right]\right.
\end{aligned}
$$


e ainda $\lambda_{m-1}^{*}=\lambda_{m}^{*}$

DEMONSTRAÇÃO:

Imediata, observando-se que pelo teorema 3.17.b, $\ell_{m-1}=\ell_{m}$.

COROLÁRIO 3.21: Se $\mu_{1}=\mu_{2}=\ldots=\mu_{m}$ temos

$$
\lambda_{j}^{*}=\frac{\lambda}{m}>0, \quad j=1,2, \ldots, m
$$

\section{DEMONSTRAÇÃO:}

Imediata, observando-se que pelo teorema 3.17.b, $\ell_{1}=\ell_{2}=\ldots=\ell_{m-1}=0$.

É simples exercício algébrico provar a seguinte generalização dos corolários acima apresentados:

Sejam $M=\{1,2, \ldots, m\}$ e $I_{1}, I_{2}, \ldots, I_{t}$ partição de $M$ tal que

i) $q \in\{1,2, \ldots, t\}, \quad \forall(i, j) \in I_{q}$

$$
\begin{aligned}
& \mu_{i}=\mu_{j}=\hat{\mu}_{q} \\
& \ell_{i-1}=\ell_{j-1}=\hat{\ell}_{q-1}
\end{aligned}
$$

ii) $q \in\{2, \ldots, t\}$

$$
\hat{\mu}_{q-1}>\hat{\mu}_{q}
$$

Temos $\left.\lambda \in] \hat{\ell}_{i-1}, \hat{\ell}_{i}\right] \Rightarrow\left[\lambda_{j}^{*}>0 \Longleftrightarrow j \leq \cup_{q \leq i} I_{q}\right] \quad i=1,2, \ldots,(t-1)$

$$
\lambda \in] \hat{\ell}_{t-1}, \mu\left[\Rightarrow\left[\lambda_{j}^{*}>0 \Longleftrightarrow j \leq m\right]\right.
$$

Por exemplo, tomemos $m=5$ e $\mu_{1}=\mu_{2}>\mu_{3}>\mu_{4}=\mu_{5}$. Temos então

$$
\begin{array}{ll}
I_{1}=\{1,2\} \quad I_{2}=\{3\} \quad I_{3}=\{4,5\} \quad \text { e } \quad \hat{\ell}_{0}=\ell_{1}=\ell_{0}, \quad \hat{\ell}_{1}=\ell_{2}, \quad \hat{\ell}_{2}=\ell_{3}=\ell_{4} \\
\left.\lambda \in] \hat{\ell}_{0}, \hat{\ell}_{1}\right] \Rightarrow \lambda_{j}^{*}>0, \quad j \in I_{1} \\
\left.\lambda \in] \hat{\ell}_{1}, \hat{\ell}_{2}\right] \Rightarrow \lambda_{j}^{*}>0, \quad j \in I_{1} \cup I_{2} \\
\lambda \in] \hat{\ell}_{2}, \mu\left[\Rightarrow \lambda_{j}^{*}>0, \quad j \in\{1,2, \ldots, 5\}\right.
\end{array}
$$

Este teorema possui uma interpretação bastante interessante: 
Podemos entender cada $\ell_{p-1}$ obtido no teorema como sendo a carga de desativação do servidor $p$, pois para cargas abaixo de $\ell_{p-1}$ o servidor $p$ não é mais utilizado uma vez que $\lambda_{p}^{*}=0$.

Portanto, a utilização ou não de um servidor é dependente da carga no sistema e não necessariamente todos os servidores devem ser sempre utilizados.

É interessante que se dois servidores tiverem mesma capacidade, eles têm a mesma carga de desativação, logo ou ambos estão ativos ou ambos estão inativos na solução ótima para uma dada carga. Lógico que se todos os servidores tiverem mesma capacidade, temos o único caso onde não há carga de desativação e todos os servidores devem estar sempre ativos, uma vez que seria absurdo todos os servidores estarem inativos para uma dada carga não nula.

\subsection{2 - Análise do $\mu$-problema}

\section{Problema da alocação ótima de capacidades nos servidores}

\subsubsection{1 - Análise para $\mathrm{N}=\mathrm{K}=\mathrm{m}$}

Para este caso, o $\mu$-problema reduz-se ao seguinte problema, a que chamaremos $P_{o}$

Dados $\lambda_{1}, \lambda_{2}, \ldots, \lambda_{m} \geq 0$

$P_{o}: \quad \min T\left(\mu_{1}, \mu_{2}, \ldots, \mu_{m}\right)$

sujeito a $\quad \sum_{i=1}^{m} \mu_{i}=\mu$

$$
\mu_{i}>\lambda_{i} \quad i=1,2, \ldots, m
$$

Esta análise consiste basicamente em determinar as capacidades $\mu_{1}^{*}, \mu_{2}^{*}, \ldots, \mu_{m}^{*}$, que são a solução de $P_{o}$.

TEOREMA 3.22: $P_{\boldsymbol{o}}$ tem solução ótima.

\section{DEMONSTRAÇÃO:}

Será omitida por ser análoga à demonstração do teorema 3.1. 
TEOREMA 3.23: A solução ótima de $\mu$-problema é dada por

$$
\mu_{i}^{*}=c_{i} * \mu+d_{i} \quad i=1,2, \ldots, m
$$

onde

$$
\begin{aligned}
c_{i} & =\frac{\sqrt{\lambda_{i}}}{\sum_{j=1}^{m} \sqrt{\lambda_{j}}} \\
d_{i} & =\lambda_{i}-\lambda \frac{\sqrt{\lambda_{i}}}{\sum_{j=1}^{m} \sqrt{\lambda_{j}}}=\frac{\lambda_{i} \sum_{\substack{j \neq i \\
j=1}}^{m} \sqrt{\lambda_{j}}-\sqrt{\lambda_{i}} \sum_{\substack{j \neq i \\
j=1}}^{m} \lambda_{j}}{\sum_{j=1}^{m} \sqrt{\lambda_{j}}}
\end{aligned}
$$

\section{DEMONSTRAÇÃO:}

As hipóteses de Lagrange verificam-se trivialmente para $P_{o}$ pois

- $\chi^{o}=\left\{z \in \mathbf{R}^{m} \mid z>\left(\lambda_{1}, \ldots, \lambda_{m}\right)\right\}$ é aberto

- $T$ é continuamente diferenciável

- $h\left(\mu_{1}, \ldots, \mu_{m}\right)=\sum_{i=1}^{m} \mu_{i}$ é continuamente diferenciável

- $\nabla h=(1,1, \ldots, 1)$

Seja então $\left(\mu_{1}^{*}, \mu_{2}^{*}, \ldots, \mu_{m}^{*}\right)$ solução ótima de $P_{o}$ então $\exists \bar{M} \in \mathrm{R} \mid\left(\mu_{1}^{*}, \mu_{2}^{*}, \ldots, \mu_{m}^{*}, \bar{M}\right)$ obedece Lagrange.

Por Lagrange temos então, para $i=1,2, \ldots, m$ :

$$
\frac{\partial T}{\partial \mu_{i}}\left(\mu_{1}^{*}, \ldots, \mu_{m}^{*}\right)=\bar{M} * \frac{\partial h}{\partial \mu_{i}}\left(\mu_{1}^{*}, \ldots, \mu_{m}^{*}\right)
$$

Mas

$$
\frac{\partial T}{\partial \mu_{i}}=\frac{-\lambda_{i}}{\lambda\left(\mu_{i}-\lambda_{i}\right)^{2}}<0, \quad e \quad \frac{\partial h}{\partial \mu_{i}}=1
$$

Portanto $M<0$ e podemos escrever que

$$
\mu_{i}^{*}=\lambda_{i}+\frac{\sqrt{\lambda_{i}}}{\sqrt{-\lambda \bar{M}}}
$$

Mas

$$
\mu=\sum_{j=1}^{m} \mu_{j}^{*}=\sum_{j=1}^{m}\left(\lambda_{j}+\frac{\sqrt{\lambda_{j}}}{\sqrt{-\lambda \bar{M}}}\right)
$$

Portanto

$$
\left.\frac{1}{\sqrt{\lambda M}}=\frac{(\mu-\lambda)}{\sum_{j=1}^{m} \sqrt{\mu j}}\right)
$$


E aplicando (2) em (1)

$$
\begin{aligned}
\mu_{i}^{*} & =\lambda_{i}+(\mu-\lambda) \frac{\sqrt{\lambda_{i}}}{\sum_{j=1}^{m} \sqrt{\lambda_{j}}} \\
& =\frac{\sqrt{\lambda_{i}}}{\sum_{j=1}^{m} \sqrt{\lambda_{j}}} * \mu+\lambda_{i}-\lambda \frac{\sqrt{\lambda_{i}}}{\sum_{j=1}^{m} \sqrt{\lambda_{j}}}
\end{aligned}
$$

Basta então definir

$$
c_{i}=\frac{\sqrt{\lambda_{i}}}{\sum_{j=1}^{m} \sqrt{\lambda_{j}}} \quad \text { e } \quad d_{i}=\lambda_{i}-\lambda \frac{\sqrt{\lambda_{i}}}{\sum_{j=1}^{m} \sqrt{\lambda_{j}}}
$$

Temos então

$$
d_{i}=\frac{\lambda_{i} \sum_{j=1}^{m} \sqrt{\lambda_{j}}-\lambda \sqrt{\lambda_{i}}}{\sum_{j=1}^{m} \sqrt{\lambda_{j}}}=\frac{\lambda_{i} \sum_{\substack{j \neq i \\ j=1}}^{m} \sqrt{\lambda_{j}}-\sqrt{\lambda_{i}} \sum_{\substack{j \neq i \\ j=1}}^{m} \lambda_{j}}{\sum_{j=1}^{m} \sqrt{\lambda_{j}}}
$$

FATO 3.23.1: $\left(\mu_{1}^{*}, \mu_{2}^{*}, \ldots, \mu_{m}^{*}\right)$ solve $P_{o}$.

\section{DEMONSTRAÇÃO:}

É fácil verificar que $\left(\mu_{1}^{*}, \mu_{2}^{*}, \ldots, \mu_{m}^{*}\right)$ é viável em $P_{o}$.

Seja $L$ o Lagrangeano definido para $P_{o}$ :

$$
\begin{aligned}
& L\left(\mu_{1}, \mu_{2}, \ldots, \mu_{m}, M\right)=\frac{1}{\lambda} \sum_{i=1}^{m} \frac{\lambda_{i}}{\mu_{i}-\lambda_{i}}+M\left(\sum_{i=1}^{m} \mu_{i}-\mu\right) \\
& \forall\left(\mu_{1}, \ldots, \mu_{m}\right) \in \chi^{0}, \quad M \in \mathbf{R}
\end{aligned}
$$

Obviamente $L$ é convexa em $\chi^{0}$, pois é soma de funções convexas.

Temos que $L\left(\mu_{1}, \mu_{2}, \ldots, \mu_{m}, M\right)=T\left(\mu_{1}, \mu_{2}, \ldots, \mu_{m}\right), \forall\left(\mu_{1}, \mu_{2}, \ldots, \mu_{m}\right) \in \chi^{0}$.

Assim,

$$
\forall\left(\mu_{1}, \mu_{2}, \ldots, \mu_{m}\right) \in \chi^{0}
$$

$T\left(\mu_{1}, \mu_{2}, \ldots, \mu_{m}\right)$

$=L\left(\mu_{1}, \mu_{2}, \ldots, \mu_{m}, \bar{M}\right)$

$\geq L\left(\mu_{1}^{*}, \ldots, \mu_{m}^{*}, \bar{M}\right)+<\nabla L\left(\mu_{1}^{*}, \mu_{2}^{*}, \ldots, \mu_{m}^{*}, \bar{M}\right),\left(\mu_{1}, \mu_{2}, \ldots, \mu_{m}\right)-\left(\mu_{1}^{*}, \mu_{2}^{*}, \ldots, \mu_{m}^{*}\right)>$ (pois L é convexa em $\chi^{0}$ )

$$
\begin{array}{cc}
=L\left(\mu_{1}^{*}, \ldots, \mu_{m}^{*}, \bar{M}\right) & \text { (pois }\left(\mu_{1}^{*}, \mu_{2}^{*}, \ldots, \mu_{m}^{*}, \bar{M}\right) \text { obedece Lagrange) } \\
=T\left(\mu_{1}, \ldots, \mu_{m}^{*}\right) & \text { (pois } \left.\left(\mu_{1}^{*}, \mu_{2}^{*}, \ldots, \mu_{m}^{*}\right) \in \chi^{0}\right)
\end{array}
$$


$\operatorname{Logo}\left(\mu_{1}^{*}, \ldots, \mu_{m}^{*}\right)$ solve $P_{o}$.

\section{Interpretação da solução obtida para o $\mu$ - problema}

A solução obtida para o $\mu$-problema é dada por

$$
\mu_{i}^{*}=\frac{\lambda_{i}}{\sum_{j=1}^{m} \sqrt{\lambda_{j}}} * \mu+\lambda_{i}-\lambda \frac{\sqrt{\lambda_{i}}}{\sum_{j=1}^{m} \sqrt{\lambda_{j}}} \quad i=1,2, \ldots, m
$$

que ainda pode ser escrita na seguinte forma

$$
\mu_{i}^{*}=\lambda_{i}+(\mu-\lambda) \frac{{\sqrt{\lambda_{i}}}^{\prime}}{\sum_{j=1}^{m} \sqrt{\lambda_{j}}}
$$

Assim, podemos dizer que a distribuição ótima da capacidade entre os servidores é obtida da seguinte forma:

- A cada servidor é atribuida a capacidade mínima para atender os jobs destinados a ele, que é dada pelo fluxo de jobs que entram pela origem a ele associada.

- A capacidade restante $(\mu-\lambda)$ é distribuída entre os servidores, na proporção das raízes quadradas dos fluxos dos jobs que entram nas origens a eles associadas.

\section{RESULTADO 3.24: Sejam $\lambda>0$ e}

$T_{p}$ o tempo médio no sistema com servidores com capacidades proporcionais à carga e $T^{*}$ o tempo médio no sistema com alocação ótima de capacidades nos servidores, então $T_{p}=T^{*}$ se e somente se a carga é a mesma em todas as origens.

\section{DEMONSTRAÇÃO:}

$$
\begin{aligned}
T_{p} & =T\left(\mu_{1}, \mu_{2}, \ldots, \mu_{m}\right)=\sum_{i=1}^{m} \frac{\lambda_{i}}{\lambda} \frac{1}{\mu_{i}-\lambda_{i}}=\sum_{i=1}^{m} \frac{\lambda_{i}}{\lambda} \frac{1}{\frac{\lambda_{i}}{\lambda} \mu-\lambda_{i}}=\frac{m}{\mu-\lambda} \\
T^{*} & =T\left(\mu_{1}^{*}, \mu_{2}^{*}, \ldots, \mu_{m}^{*}\right) \\
& =\sum_{i=1}^{m} \frac{\lambda_{i}}{\lambda} \frac{1}{\lambda_{i}+(\mu-\lambda) \frac{\sqrt{\lambda_{i}}}{\sum_{j=1}^{m} \sqrt{\lambda_{j}}}-\lambda_{i}}=\frac{1}{\lambda(\mu-\lambda)}\left(\sum_{j=1}^{m} \sqrt{\lambda_{j}}\right)^{2} \\
T_{p} & \geq T^{*} \Longleftrightarrow \lambda_{m} \geq\left(\sum_{j=1}^{m} \sqrt{\lambda_{j}}\right)^{2}
\end{aligned}
$$


Sejam $a=(1,1, \ldots, 1) \in \mathbf{R}_{m} \quad$ e $\quad b=\left(\sqrt{\lambda_{1}}, \ldots, \sqrt{\lambda_{m}}\right) \in \mathbf{R}_{m}$, pela desigualdade de Cauchy-Schwartz, vem que

$$
\begin{aligned}
\left(\sum_{j=1}^{m} a_{j} b_{j}\right)^{2} & \leq\left(\sum_{j=1}^{m} a_{j}^{2}\right)\left(\sum_{j=1}^{m} b_{j}^{2}\right) \\
\left(\sum_{j=1}^{m} 1 \cdot \sqrt{\lambda_{j}}\right)^{2} & \leq \sum_{j=1}^{m} 1^{2} \cdot \sum_{j=1}^{m}\left(\sqrt{\lambda_{j}}\right)^{2} \\
\left(\sum_{j=1}^{m} \sqrt{\lambda_{j}}\right)^{2} & \leq \lambda \cdot m \\
T^{*} & \leq T_{p}
\end{aligned}
$$

A igualdade vale se $a$ e $b$ são vetores L.D., ou seja, se $\lambda_{1}=\lambda_{2}=\ldots=\lambda_{m}$.

Por outro lado, se $\lambda_{1}=\lambda_{2}=\ldots=\lambda_{m}$ é imediato que $T_{p}=T^{*}$.

Mas, para $\lambda_{1}=\lambda_{2}=\ldots=\lambda_{m}$, temos $\mu_{1}^{*}=\mu_{2}^{*}=\ldots=\mu_{m}^{*}$.

Assim, esse resultado nos mostra que a alocação ótima de capacidades nos servidores consiste de dividir a capacidade total igualmente entre os servidores somente se a carga total estiver dividida igualmente entre as origens.

\subsection{3 - Alocação de serviço $\times$ alocação de capacidades:}

Comparação entre o $\lambda$-problema e o $\mu$-problema

Consideremos o sistema $S(\lambda, \mu, 2,2)$.

Seja a função $P$, que define a razão entre as cargas ótimas nas duas origens, dada por

$P(\lambda)=\frac{\lambda_{1}^{*}(\lambda)}{\lambda_{2}^{*}(\lambda)}, \quad \lambda>\bar{\lambda}$

onde $\left\{\lambda_{i}^{*}\right\}$ é a solução do $\lambda$-problema associado, dados $\mu_{1}, \mu_{2} \operatorname{com} \mu_{1}>\mu_{2}>0$

De forma análoga ao resultado 3.8 , podemos mostrar que:

a) $P(\lambda)$ é decrescente $\operatorname{com} \lambda$

b) $P(\lambda)$ é convexa em $\lambda$

c) $\lim _{\lambda \rightarrow \mu} p(\lambda)=\frac{\mu_{1}}{\mu_{2}}$ 
Seja a função $Q$, que define a razão entre as capacidades ótimas nos dois servidores, dada por

$$
Q(\mu)=\frac{\mu_{1}^{*}(\mu)}{\mu_{2}^{*}(\mu)}
$$

onde $\left\{\mu_{i}^{*}\right\}$ é a solução do $\mu$-problema associado, dados $\lambda_{1}, \lambda_{2} \operatorname{com} \lambda_{1}>\lambda_{2}$

De forma análoga ao resultado 3.8 , podemos mostrar que:

a) $Q(\mu)$ é descrescente com $\mu$

b) $Q(\mu)$ é convexa em $\mu$

c) $\lim _{\mu \rightarrow \infty} Q(\mu)=\frac{\sqrt{\lambda_{1}}}{\sqrt{\lambda_{2}}} \Longleftrightarrow \rho \rightarrow 0 \Rightarrow Q(\mu) \rightarrow \frac{\sqrt{\lambda_{1}}}{\sqrt{\lambda_{2}}}$

$\lim _{\mu \rightarrow \lambda} Q(\mu)=\frac{\lambda_{1}}{\lambda_{2}} \Longleftrightarrow \rho \rightarrow 1 \Rightarrow Q(\mu) \rightarrow \frac{\lambda_{1}}{\lambda_{2}}$

Assim, temos os seguintes resultados gráficos
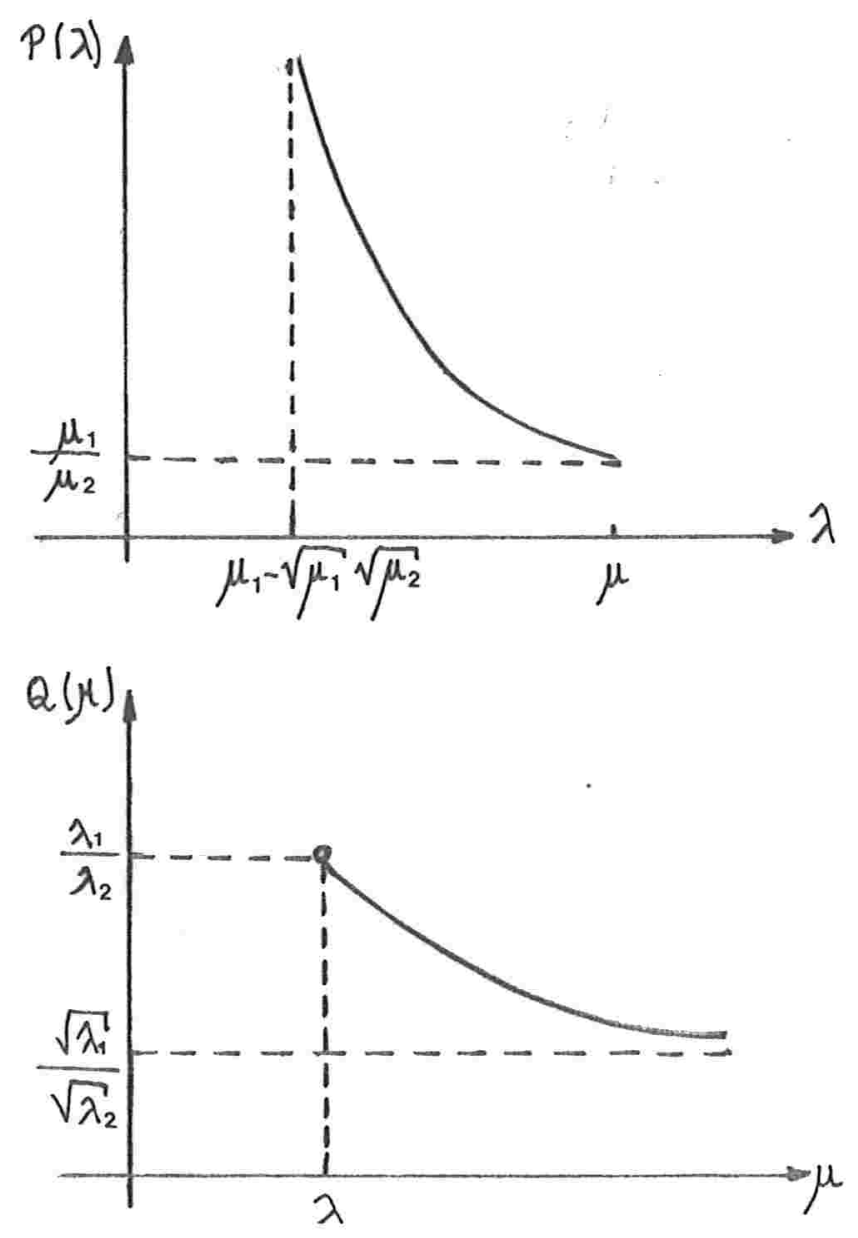

$\mu$-PROBLEMA

$\left(\lambda_{1}, \lambda_{2}\right.$ dados, $\left.\lambda_{1}>\lambda_{2}\right)$ 


\section{OBSERVAÇÃO:}

Para o $\lambda$-problema mostramos através do teorema 3.7 , que a solução ótima é dada em função da carga total do sistema, sendo que num intervalo de carga ela é dada pela concentração da carga total no servidor 'mais forte' (primeiro servidor) e no outro intervalo ela é dada por Lagrange.

Já para o $\mu$-problema a solução ótima é sempre dada por Lagrange.

Estes fatos indicam que o $\lambda$-problema e o $\mu$-problema não são problemas duais.

\subsection{4 - A nálise do $(\lambda, \mu)$-problema}

\section{Problema da alocação ótima de cargas e capacidades}

O teorema apresentado a seguir, adaptado a partir de um resultado de [15], diz respeito à solução ótima do $(\lambda, \mu)$ - problema generalizado, que consiste do $(\lambda, \mu)$-problema já definido, com restrições de fluxo mínimo de jobs que devem entrar por cada uma das origens, que são dadas por:

$$
\lambda_{i} \geq K_{i} \quad i \in M=\{1,2, \ldots, m\}
$$

onde

$$
K_{1} \geq K_{2} \geq \ldots \geq K_{m} \quad \text { e } \quad \sum_{i=1}^{m} K_{i} \leq \lambda
$$

$\mathrm{O}(\lambda, \mu)$-problema generalizado reduz-se ao seguinte problema a que chamaremos $P_{o}$ $P_{o}: \quad \min T\left(\lambda_{I}, 0_{M-I}, \mu_{I}, 0_{M-I}\right)$

sujeito a $\sum_{i \in I} \lambda_{i}=\lambda$

$$
\begin{aligned}
& \sum_{i \in I} \lambda_{i}=\lambda \\
& \sum_{j \in I} \mu_{j}=\mu \\
& K_{i} \leq \lambda_{i}<\mu_{i} \quad i \in I
\end{aligned}
$$

$I \subseteq M$

Estamos considerando $\lambda_{j}=\mu_{j}=0, j \in M-I$, onde $M-I=\{j \in M$ e $j \notin I\}$. Para isto ser possivel temos que ter $K_{j}=0, j \in M-I$. Assim, o problema definido permite que alguns servidores seja eliminados na solução ótima. 
TEOREMA 3.25: A solução do $(\lambda, \mu)$-problema generalizado é dada por

$$
\begin{gathered}
\lambda_{i}^{*}= \begin{cases}\lambda-\sum_{j=1}^{m} K_{j} & \text { se } i=1 \\
K_{i}, & \text { se } 2 \leq i \leq m\end{cases} \\
\mu_{i}^{*}=\lambda_{i}^{*}+(\mu-\lambda) \frac{\sqrt{\lambda_{i}^{*}}}{\sum_{j=1}^{m} \sqrt{\lambda_{j}^{*}}} \quad i=1,2, \ldots, m
\end{gathered}
$$

e o valor mínimo para tempo médio no sistema é

$$
\frac{\left(\sum_{i=1}^{m} \sqrt{\lambda_{i}^{*}}\right)^{2}}{\lambda(\mu-\lambda)}
$$

DEMONSTRAÇÃO:

FATO 3.25.1: $P_{o}$ tem solução ótima.

\section{DEMONSTRAÇÃO:}

O problema $P_{o}$ considera na verdade todos os possíveis subconjuntos de $M$ com as características apresentadas. Vamos então considerar um deles, o conjunto $J \subseteq M$.

O conjunto definido por

$$
B_{1}=\left\{\left(\lambda_{1}, \lambda_{2}, \ldots, \lambda_{m}\right) \in \mathbf{R}^{m} \mid \sum_{i \in J} \lambda_{i}=\lambda, \quad \lambda_{i} \geq K_{i} \text { para } i \in J, \quad \lambda_{j}=0 \text { para } j \notin J\right\}
$$

é compacto.

Para a restrição $\mu_{i}>\lambda_{i} \quad i \in J$, temos que analogamente ao apresentado no teorema $3.1, \exists \delta_{i} \in \mathbf{R}, 0<\delta_{i}<1$, tal que podemos reescrevê-la sem alterar a solução ótima, na forma $\mu_{i} \geq \delta_{i} \lambda_{i}$. Assim, podemos consideràr o conjunto

$$
\begin{gathered}
B_{2}=\left\{\left(\mu_{1}, \mu_{2}, \ldots, \mu_{m}\right) \in \mathbf{R}^{m} \mid \sum_{i \in J} \mu_{i}=\mu, \mu_{i} \geq \delta_{i} \lambda_{i} \text { para } i \in J \text { e } \lambda_{i} \in B_{1},\right. \\
\left.\mu_{j}=0 \text { para } j \notin J\right\}
\end{gathered}
$$

e temos que $B_{2}$ é compacto.

Como a função é contínua em $B_{1} \times B_{2}$ temos que existe solução ótima para o problema $P_{o}$ considerando o conjunto $J$. 
Mas existe um número finito de possíveis subconjuntos de $M$, logo basta escolher o subconjunto $J^{*}$ que fornece o menor valor para a função $T$ e obtemos a solução de $P_{o}$.

FATO 3.25.2: O problema $P_{o}$ é equivalente ao seguinte problema:

$$
\begin{aligned}
P_{p}: \quad \min & G\left(\lambda_{I}, 0_{M-I}\right) \\
\text { sujeito a } & \sum_{i \in I} \lambda_{i}=\lambda \\
& \lambda_{i} \geq K_{i} \quad i \in I
\end{aligned}
$$

onde

$G\left(\lambda_{I}, 0_{M-I}\right)=T\left(\lambda_{I}, \mu_{I}^{*}\left(\lambda_{I}, 0_{M-I}\right), \mu_{M-I}^{*}\left(\lambda_{I}, 0_{M-I}\right)\right)$

$\mu_{i}^{*}$-capacidade ótima no i-ésimo servidor, dada pela solução do $\mu$-problema

\section{DEMONSTRAÇÃO:}

A demonstração é trivial notando que o problema $P_{p}$ é obtido pela técnica de decomposição e projeção (vide [09] e [13]).

É conveniente observar que a restrição $\lambda_{i}<\mu_{i}^{*}$ não precisa ser incluída, pois o $\mu-$ problema projetado a contém.

Pelos Fatos 3.25.1 e 3.25.2 temos que para obter a solução do problema $P_{o}$ é suficiente obter a solução do problema $P_{p}$.

A função $G$ é definida por

$$
\begin{aligned}
G\left(\lambda_{1}, \ldots, \lambda_{m}\right) & =T\left(\lambda_{1}, \lambda_{2}, \ldots, \lambda_{m}, \mu_{1}^{*},\left(\lambda_{1}, \ldots, \lambda_{m}\right), \ldots, \mu_{m}^{*}\left(\lambda_{1}, \ldots, \lambda_{m}\right)\right) \\
& =\sum_{i=1}^{m} \frac{\lambda_{i}}{\lambda} \frac{1}{\mu_{i}^{*}\left(\lambda_{1}, \ldots, \lambda_{m}\right)-\lambda_{i}}=\sum_{i=1}^{m} \frac{\lambda_{i}}{\lambda} \frac{1}{(\mu-\lambda) \frac{\sqrt{\lambda_{i}}}{\sum_{j=1}^{m} \sqrt{\lambda_{j}}}} \\
& =\frac{1}{\lambda(\mu-\lambda)}\left(\sum_{i=1}^{m} \sqrt{\lambda_{i}}\right)^{2}
\end{aligned}
$$

Logo, minimizar $G$ é equivalente a minimizar $S\left(\lambda_{1}, \lambda_{2}, \ldots, \lambda_{m}\right)=\sum_{i=1}^{m} \sqrt{\lambda_{i}}$. Vamos obter a solução por um processo construtivo a partir de $I=M$.

\section{Processo construtivo da solução:}

Seja $\left(\lambda_{1}, \ldots, \lambda_{m}\right)$ um ponto viável para o problema $P_{p}$.

Definamos $J=\left\{j \in\{2, \ldots, m\} \mid \lambda_{j}>K_{j}\right\}$.

Seja $j \in J$ 


$$
\lambda_{j}>K_{j} \Rightarrow \lambda_{j}=K_{j}+\alpha_{j} \quad \text { com } \quad \alpha_{j}>0
$$

Vamos então definir o ponto $\left(\lambda_{1}^{\prime}, \ldots, \lambda_{m}^{\prime}\right)$ tal que

$$
\begin{aligned}
& \lambda_{j}^{\prime}=K_{j} \\
& \lambda_{1}^{\prime}=\lambda_{1}+\alpha_{j} \\
& \lambda_{i}^{\prime}=\lambda_{i} \quad 2 \leq i \leq m, i \neq j
\end{aligned}
$$

Temos:

a) $\left(\lambda_{1}^{\prime}, \ldots, \lambda_{m}^{\prime}\right)$ é viável.

$$
\begin{aligned}
& \sum_{i=1}^{m} \lambda_{i}^{\prime}=\lambda_{1}^{\prime}+\lambda_{j}^{\prime}+\sum_{\substack{i \neq j \\
i=2}}^{m} \lambda_{i}^{\prime}=\left(\lambda_{1}+\alpha_{j}\right)+K_{j}+\sum_{\substack{i \neq j \\
i=2}}^{m} \lambda_{i}=\sum_{i=1}^{m} \lambda_{i}=\lambda \\
& \lambda_{1}^{\prime}=\lambda_{1}+\alpha_{j}>\lambda_{1} \geq K_{1} \\
& \lambda_{j}^{\prime}=K_{j} \\
& \lambda_{i}^{\prime}=\lambda_{i} \geq K_{i} \quad 2 \leq i \leq m, i \neq j
\end{aligned}
$$

b) $S\left(\lambda_{1}^{\prime}, \lambda_{2}^{\prime}, \ldots, \lambda_{m}^{\prime}\right) \leq S\left(\lambda_{1}, \lambda_{2}, \ldots, \lambda_{m}\right)$

$$
\begin{aligned}
& \Longleftrightarrow \sqrt{\lambda_{1}^{\prime}}+\sqrt{\lambda_{j}^{\prime}}+\sum_{\substack{i \neq j \\
i=2}}^{m} \sqrt{\lambda_{i}^{\prime}} \leq \sqrt{\lambda_{1}}+\sqrt{\lambda_{j}}+\sum_{\substack{i \neq j \\
i=2}}^{m} \sqrt{\lambda_{i}} \\
& \Longleftrightarrow \sqrt{\lambda_{1}+\alpha_{j}}+\sqrt{K_{j}} \leq \sqrt{\lambda_{1}}+\sqrt{K_{j}+\alpha_{j}} \\
& \Longleftrightarrow \lambda_{1}+\alpha_{j}+K_{j}+2 \sqrt{\lambda_{1}+\alpha_{j}} \sqrt{K_{j}} \leq \lambda_{1}+K_{j}+\alpha_{j}+2 \sqrt{\lambda_{1}} \sqrt{K_{j}+\alpha_{j}} \\
& \Longleftrightarrow\left(\lambda_{1}+\alpha_{j}\right) K_{j} \leq \lambda_{1}\left(K_{j}+\alpha_{j}\right) \\
& \Longleftrightarrow K_{j} \leq \lambda_{1} \quad \text { pois } \quad \alpha_{j}>0
\end{aligned}
$$

Mas $K_{j} \leq K_{1} \leq \lambda_{1}$ portanto $S\left(\lambda_{1}^{\prime}, \ldots, \lambda_{m}^{\prime}\right) \leq S\left(\lambda_{1}, \ldots, \lambda_{m}\right)$

Fazemos $J=J-\{j\}$ e repetimos o processo acima até que $J=\emptyset$, tomando para cada repetição o $\left(\lambda_{1}^{\prime}, \ldots, \lambda_{m}^{\prime}\right)$ obtido no passo anterior.

Obtemos $\lambda^{*}=\left(\lambda_{1}^{*}, \lambda_{2}^{*}, \ldots, \lambda_{m}^{*}\right)$, que é o último ponto viável resultante da aplicação do processo (pode ser $\left(\lambda_{1}, \ldots, \lambda_{m}\right)$, caso $J=\emptyset$ no início do processo) e vale que:

$$
\begin{aligned}
& \lambda_{i}^{*}=K_{i} \quad i=1,2, \ldots, m \\
& \lambda_{i}^{*}=\lambda-\sum_{i=2}^{m} \lambda_{i}^{*}=\lambda-\sum_{i=2}^{m} K_{i}
\end{aligned}
$$


Resta provar que $\lambda^{*}$ é a solução do problema $P_{p}$.

Seja $\bar{\lambda}=\left(\bar{\lambda}_{1}, \ldots, \bar{\lambda}_{m}\right)$ solução ótima para o problema $P_{p}$ e vamos supor $\bar{\lambda} \neq \lambda^{*}$.

FATO 3.25.3: Se existir mais de uma componente $j$ tal que $\lambda_{j}^{i}>K_{j}$ então

$$
S\left(\lambda^{i}\right)<S\left(\lambda^{f}\right)
$$

onde

$\lambda^{i}=\left(\lambda_{1}^{i}, \ldots, \lambda_{m}^{i}\right)$ é o ponto viável inicial para o processo e

$\lambda^{f}=\left(\lambda_{1}^{f}, \ldots, \lambda_{m}^{f}\right)$ é o ponto final obtido pelo processo.

\section{DEMONSTRAÇÃO:}

Vamos mostrar que a partir da 2 a. repetição do processo, não pode ocorrer da função $S$ não ter decrescido.

Sejam $j$ e $\ell$ tal que $\lambda_{j}^{i}>K_{j}$ e $\lambda_{\ell}^{i}>K_{\ell}$ e $J=\left\{p \in\{1,2, \ldots, m\} \mid \lambda_{p}^{i}>K_{p}\right\}$. Aplicando o processo temos

$$
\begin{aligned}
& \lambda_{1}^{\prime}=\lambda_{1}^{i}+\lambda_{j}^{i}-K_{j} \\
& \lambda_{j}^{\prime}=K_{j} \\
& \lambda_{p}^{\prime}=\lambda_{p}^{i} \quad 2 \leq p \leq m, \quad p \neq j \\
& \text { e } S\left(\lambda^{\prime}\right) \leq S\left(\lambda^{i}\right)
\end{aligned}
$$

Supondo $S\left(\lambda^{\prime}\right)=S\left(\lambda^{i}\right)$ temos $K_{j}=\lambda_{1}^{i}$

Mas $K_{1} \leq \lambda_{1}^{i}$ e $K_{1} \geq K_{j}$, portanto $K_{1}=K_{j}$

$\ell \in J$ e aplicando novamente o processo temos

$$
\begin{aligned}
& \lambda_{1}^{\prime \prime}=\lambda_{1}^{\prime}+\lambda_{\ell}^{\prime}-K_{\ell} \\
& \lambda_{\ell}^{\prime \prime}=K_{\ell} . \\
& \lambda_{p}^{\prime \prime}=\lambda_{p}^{\prime}, \quad 2 \leq p \leq m, \quad p \neq \ell \\
& \text { e } S\left(\lambda^{\prime \prime}\right) \leq S\left(\lambda^{\prime}\right)
\end{aligned}
$$

$S\left(\lambda^{\prime \prime}\right)=S\left(\lambda^{\prime}\right) \Rightarrow K_{\ell}=\lambda_{1}^{\prime}$

Mas $K_{1} \leq \lambda_{1}^{\prime}$ e $K_{1} \geq K_{\ell}$ portanto $K_{1}=K_{\ell}$

(2) e (4) implicam em $K_{\ell}=K_{j}$.

Mas $K_{\ell} \stackrel{(3)}{=} \lambda_{1}^{\prime} \stackrel{(1)}{=} \lambda_{j}^{i} \quad$ e $\quad K_{\ell}=K_{j} \Rightarrow \lambda_{j}^{i}=K_{j}$, o que é absurdo. 
FATO 3.25.4: $\exists 2 \leq j \leq m: \bar{\lambda}_{j}>\lambda_{j}^{*}$.

\section{DEMONSTRAÇÃO:}

$\bar{\lambda} \neq \lambda^{*} \Rightarrow \exists \ell$ tal que $\bar{\lambda}_{\ell} \neq \lambda_{\ell}^{*}$

Se $\ell=1$ temos que

$$
\text { se } \bar{\lambda}_{\ell}>\lambda_{\ell}^{*} \Rightarrow \exists j \geq 2 \text { tal que } \bar{\lambda}_{j}<\lambda_{j}^{*} \text { pois } \sum_{i=1}^{m} \bar{\lambda}_{i}=\lambda
$$

o que é absurdo pois $\bar{\lambda}_{j} \geq K_{j}=\lambda_{j}^{*}$.

Logo $\bar{\lambda}_{\ell}<\lambda_{\ell}^{*}$, o que implica que $\exists j \geq 2$ tal que $\bar{\lambda}_{j}>\lambda_{j}^{*}$.

Se $2 \leq \ell \leq m$ temos que $\bar{\lambda}_{\ell}>\lambda_{\ell}^{*}$ pois $\bar{\lambda}_{\ell} \geq K_{\ell}=\lambda_{\ell}^{*}$ e basta tomar $j=\ell$.

Portanto, pelo Fato 3.25 .4 , existe $2 \leq j \leq m$ tal que $\bar{\lambda}_{j}>\lambda_{j}^{*}=K_{j}$.

Aplicando o Fato 3.25 .3 temos que $j$ é a única componente pois $\bar{\lambda}$ é ótimo.

Logo

$$
\begin{aligned}
& \bar{\lambda}_{p}=\lambda_{p}^{*} \quad 1 \leq p \leq m, \quad p \neq j \\
& \bar{\lambda}_{j}>\lambda_{j}^{*}
\end{aligned}
$$

Mas

$$
\begin{gathered}
\sum_{p=1}^{m} \bar{\lambda}_{p}=\sum_{\substack{p \neq j \\
p=1}}^{m} \bar{\lambda}_{p}+\bar{\lambda}_{j}=\sum_{\substack{p \neq j \\
p=1}}^{m} \lambda_{p}^{*}+\bar{\lambda}_{j}=\lambda \\
\sum_{p=1}^{m} \lambda_{p}^{*}=\sum_{\substack{p \neq j \\
p=1}}^{m} \lambda_{p}^{*}+\lambda_{j}^{*}=\lambda \\
\Rightarrow \bar{\lambda}_{j}=\lambda_{j}^{*}, \quad \text { o que é absurdo }
\end{gathered}
$$

Logo, $\bar{\lambda}=\lambda^{*}$.

Portanto $\lambda^{*}$ é a solução ótima para o problema $P_{p}$.

$\mathrm{E}\left(\lambda_{1}^{*}, \ldots, \lambda_{m}^{*}, \mu_{1}^{*}, \ldots, \mu_{m}^{*}\right)$ é solução ótima para o $(\lambda, \mu)$-problema modificado, com

$$
\mu_{i}^{*}=\lambda_{i}^{*}+(\mu-\lambda) \frac{\sqrt{\lambda_{i}^{*}}}{\sum_{j=1}^{m} \sqrt{\lambda_{j}^{*}}}
$$

COROLÁRIO 3.26: Se $K_{1}=K_{2}=\ldots=K_{m}=0$ temos

$$
\begin{array}{ll}
\lambda_{1}^{*}=\lambda, & \lambda_{2}^{*}=\ldots=\lambda_{m}^{*}=0 \\
\mu_{1}^{*}=\mu, & \mu_{2}^{*}=\ldots=\mu_{m}^{*}=0
\end{array}
$$




\section{DEMONSTRAÇÃO:}

Imediata, a partir das expressões obtidas para $\lambda_{i}^{*}$ e $\mu_{i}^{*}$, notando que $\lambda_{i}<\mu_{i} \Longleftrightarrow$ $\mu_{i}>0 \quad$ e $\quad \lambda_{i}=0$ se $\mu_{i}=0$.

Este corolário diz respeito à não exigência de fluxo mínimo de jobs entrando pelas origens, e mostra que o melhor desempenho é obtido para o sistema quando concentramos toda a capacidade num só servidor. Obviamente, a capacidade concentrada num só servidor só é possivel quando a carga também estiver concentrada na origem correspondente ao servidor, uma vez que os demais servidores e seus respectivos grupos são eliminados.

\subsection{5 - Conclusão}

Pode-se dizer que um sistema distribuído tem um desempenho ótimo quando todos os recursos disponíveis no sistema estão sendo bem utilizados.

O projeto de um sistema distribuído deve considerar conjuntamente a melhor forma de distribuir a carga do sistema entre os servidores e a distribuição da capacidade de atendimento desses servidores.

Quando o sistema já está em produção devem ser feitas medidas de seu desempenho, a fim de verificar se o sistema está sendo bem utilizado e em caso contrário devem ser feitos ajustes, por exemplo uma redistribuição de carga, de modo a maximizar a utilização do investimento feito no sistema atual.

Caso o sistema não esteja fornecendo o tempo de resposta desejado, mesmo considerando-se a melhor utilização possível do mesmo, torna-se necessário aumentar a capacidade do sistema atual, ou seja, são necessárias evoluções de hardware.

Nosso estudo considerou como principal recurso a capacidade de processamento (CPU). O projeto do nosso sistema de partição de servidores (PS) pode ser modelado pelo $(\lambda, \mu)$-problema, o ajuste de desempenho pode ser modelado pelo $\lambda$-problema e o ajuste de capacidade (evolução) pode ser modelado pelo $\mu$-problema.

Dois resultados importantes foram obtidos: o resultado que determina a distribuição ótima da carga do sistema (teorema 3.18) e o que determina que se considerarmos que o custo de qualquer servidor é proporcional à sua capacidade (custo servidor ${ }_{j}=$ constante ${ }^{*} \mu_{j}$ ) e que o fluxo mínimo de jobs em qualquer origem é nulo, é melhor 
concentrarmos a capacidade de atendimento num só servidor (corolário 3.26). Esses resultados foram obtidos para uma partição com grupos de apenas um servidor.

No próximo capítulo trataremos de grupos com $m$ servidores $(m \geq 2)$, a fim de obtermos mais parâmetros para aprofundar a análise do algoritmo PS. 


\section{Capítulo 4}

Partição Não Singular de Servidores

Este capítulo tem por função estender o estudo do algoritmo partição dos servidores para partições não singulares, isto é, partições com grupos não singulares, que são grupos com mais do que um servidor.

Tal o faremos através do estudo do comportamento de um fluxo de serviços sendo atendido por um grupo de $m$ servidores cuja capacidade total de atendimento é constante. Assim consideraremos o grupo como um sistema onde todos os jobs que entram para serem processados juntam-se numa fila comum de espera e são processados por $m$ servidores heterogêneos, isto é, por servidores com capacidades de atendimento distintas. Esse sistema é também chamado sistema heterogêneo. 


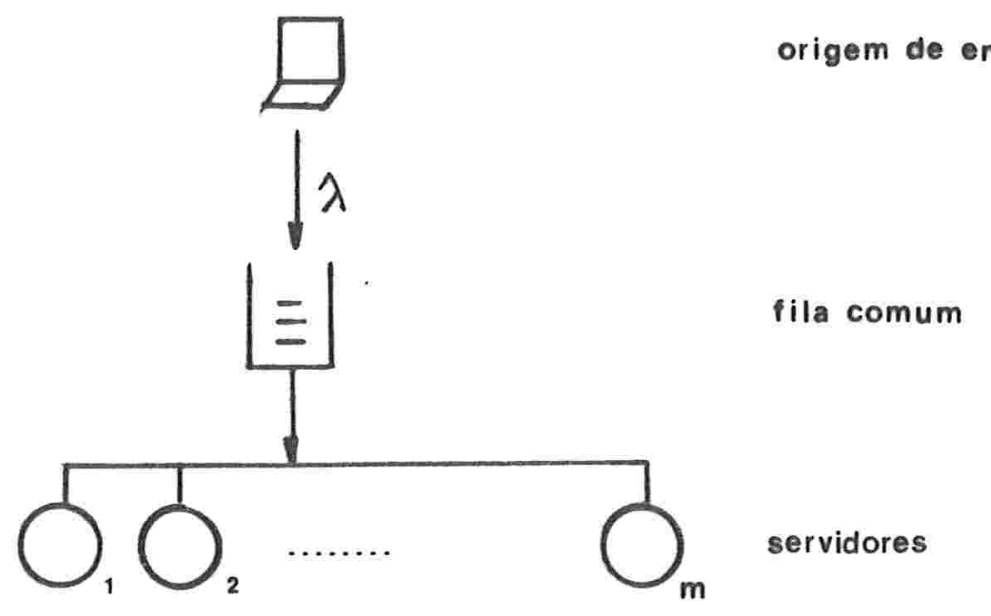

Considerando-se que a chegada dos jobs no sistema tem distribuição Poisson e que os servidores têm tempo de serviço com distribuição exponencial, podemos dizer que o sistema acima corresponde a um sistema de fila Markoviano do tipo $\mathrm{M} / \mathrm{M} / \mathrm{m}$, e pode ser denotado por $S(\lambda, \mu, 1, m)$.

O sistema $M / M / m$ com servidores homogêneos, ou seja, servidores com mesma capacidade de atendimento, também chamado sistema homogêneo, já foi estudado e para ele já existe na literatura, (vide [16], [30]), expressões para tempo médio no sistema ( $t s)$ e para tempo médio na fila do sistema $\left(t_{f}\right)$.

O estudo de sistemas heterogêneos apresentado neste capítulo concentra-se basicamente em:

- determinação de expressões para $t_{s}$ e $t_{f}$;

- obtenção do projeto ótimo do sistema de serviço em relação à $t_{s}$, ou seja, determinação da distribuição de capacidades entre os servidores que fornece o menor $t_{s}$;

- obtenção do projeto ótimo do sistema de serviço em relação à $t_{f}$;

- análise comparativa entre sistemas $S(\lambda, \mu, 1, m)$ homogêneos e heterogêneos;

- análise comparativa entre sistemas $S(\lambda, \mu, 1, m)$ e o correspondente $S(\lambda, \mu, 1,1)$, sendo que dizemos que o sistema $S(\lambda, \mu, 1,1)$ é correspondente ao sistema $S(\lambda, \mu, 1, m)$ pois é um sistema de um servidor com mesma carga total e mesma capacidade total de atendimento que o sistema de $m$ servidores.

Inicialmente apresentamos o estudo para o caso $m=2$, que já foi anteriormente estudado por Singh (em [23]) e Nishida (em [20] e [25]) em contextos um pouco diferentes, 
pois em [20] e [25] considerou-se que jobs que chegam no sistema e encontram os servidores ocupados não esperam pelo atendimento, e em [23] considerou-se que jobs que chegam no sistema e encontram os servidores ocupados esperam com uma certa probabilidade pelo atendimento. Os resultados analíticos e gráficos apresentados tentam abranger e ampliar os resultados por eles obtidos nos seus contextos.

Para o caso $m=3$ apresentamos um estudo menos completo, mostrando basicamente expressões para tempo médio no sistema e para tempo médio na fila do sistema.

Fechamos o capítulo com a comparação de processamento centralizado e distribuído, considerando partição de servidores.

\section{1 - Especificação completa do grupo não singular}

O sistema $S(\lambda, \mu, 1, m), \mu>0$, por nós considerado, possui as seguintes características:

(a) processo de chegada dos jobs no sistema:

Um job que entra no sistema por qualquer origem é introduzido no final de uma fila comum de espera. O processo de chegada de jobs na fila tem uma distribuição Poisson com média $\lambda$.

(b) processo de atendimento:

Os tempos de atendimento de cada um dos servidores são independentes e distribuídos exponencialmente, sendo que

(b.1) $\mu_{i}^{-1}$ é o tempo médio de atendimento do i-ésimo servidor, $i=1, \ldots, m$ (b.2) $\mu_{1} \geq \mu_{2} \geq \ldots \geq \mu_{m}$, ou seja, o primeiro servidor é pelo menos tão rápido quando o segundo, que por sua vez é pelo menos tão rápido quanto o terceiro e assim por diante.

(b.3) $\mu_{1}+\mu_{2}+\ldots+\mu_{m}=\mu$

(c) disciplina na fila:

Um job que chega no sistema pode encontrar as seguintes situações:

(c.1) pelo menos dois servidores livres - neste caso, o job vai ser atendido (processado) pelo servidor livre que seja mais rápido. Esta regra é denominada FSR ('fastest service rule'). 
(c.2) apenas um servidor livre - neste caso, o job vai ser atendido pelo servidor livre.

(c.3) nenhum servidor livre - neste caso, o job entra no fim da fila de espera. O job que está no início da fila ocupará o servidor que se tornar livre primeiro, logo, temos a disciplina FCFS para atendimento. Vamos considerar a fila com capacidade infinita, ou seja, não existe limite para número de jobs na fila.

Este item vem a ser a diferença básica dos estudos feitos em [23], [20] e [25].

Podemos apresentar esquematicamente a fila $\mathrm{M} / \mathrm{M} / \mathrm{m}$, correspondente ao sistema $S(\lambda, \mu, 1, m)$, conforme abaixo:

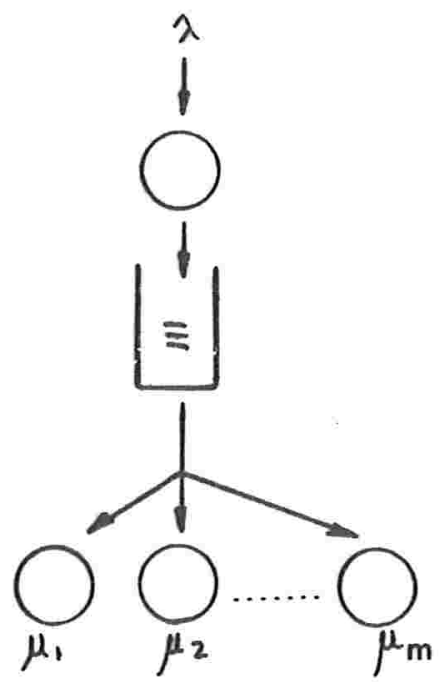

Para obtenção de $T_{s}$ e $T_{f}$ é necessário formular o diagrama de transição de estados para esta fila, e para tanto são introduzidas as seguintes definições:

- Os estados do sistema são representados por tuplas $\left(x_{1}, x_{2}, \ldots, x_{m}, x_{f}\right)$ onde $x_{j}$ é o número de jobs no servidor $j \quad\left(x_{j} \in\{0,1\} \quad j=1, \ldots, m\right)$ e $\quad x_{f}$ é o número de jobs na fila do sistema $\left(x_{f} \geq 0\right)$. Vamos denotar por $E_{i}^{(j)}$ a tupla $\left(x_{1}^{(j)}, x_{2}^{(j)}, \ldots, x_{m}^{(j)}, x_{f}\right)$ onde $x_{1}^{(j)}+x_{2}^{(j)}+\ldots+x_{m}^{(j)}+x_{f}=i$

Assim podemos denotar por $E_{i}$, ao 'estado' onde o sistema possui $i$ jobs, sendo que $E_{i}=\left\{E_{i}^{(j)}\right.$, para todos $j$ possíveis $\}$. 
Os 'estados' $E_{i}, i \geq m$, são representados apenas pela tupla $(1,1, \ldots, 1, i-m)$. Vale notar que o diagrama de transição de estados obtido para esses estados é o diagrama de um processo de nascimento e morte, cujo tratamento é conhecido na literatura ([16], [06]).

- $p_{i}$ é a probabilidade de existirem $i$ jobs no sistema em equilíbrio. O sistema é dito em equilíbrio quando existe conservação de fluxo, ou seja, quando o fluxo de entrada é igual ao fluxo de saída em cada estado.

$$
\begin{gathered}
p_{i j} \triangleq \text { probabilidade }\left(E_{i}^{(j)}\right) \\
p_{i} \triangleq \text { probabilidade }\left(E_{i}\right)=\sum_{j} p_{i j} \\
\sum_{i=0}^{\infty} p_{i}=1
\end{gathered}
$$

Vamos considerar as seguintes notações:

$T_{s}^{m}$ - tempo médio no sistema $S(\lambda, \mu, 1, m), m \geq 1$.

$T_{f}^{m}$ - tempo médio na fila do sistema $S(\lambda, \mu, 1, m), m \geq 1$.

Vale lembrar que o fator de utilização global do sistema $S(\lambda, \mu, 1, m)$ é dado por:

$$
\rho=\frac{\lambda}{\mu}<1
$$

\section{2 - Grupo de dois servidores}

O diagrama de transição de estados para o sistema $S(\lambda, \mu, 1,2)$ tem a seguinte representação 


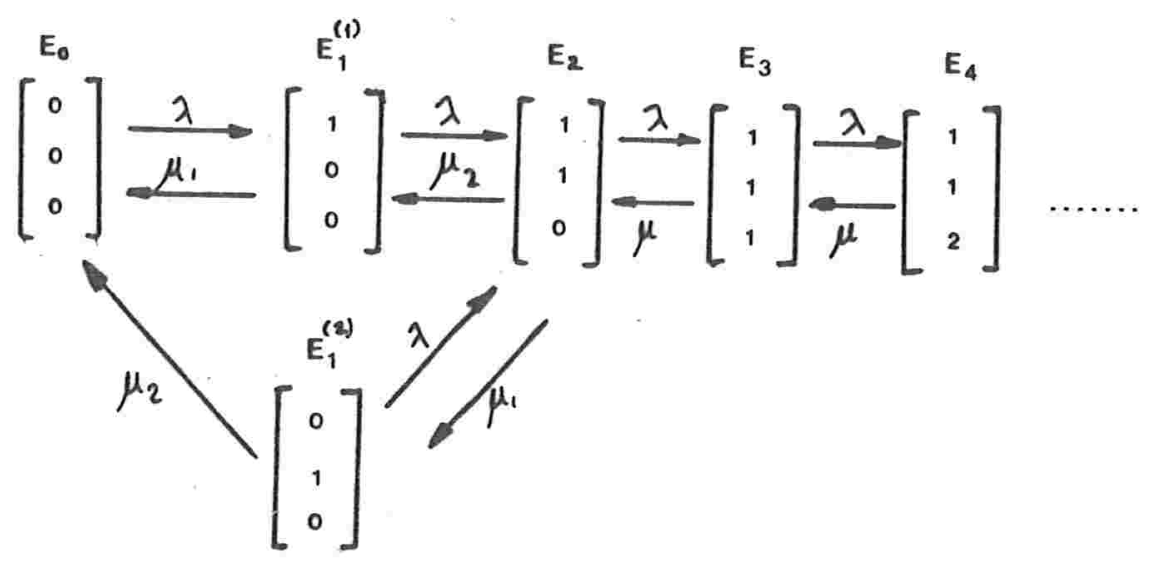

\section{OBSERVAÇÕES:}

1. A notação $E_{1}^{(j)}$ é utilizada, pois existe mais de uma possibilidade de termos um elemento no sistema. Temos, na verdade,

$$
\begin{aligned}
p_{1} & =\text { probabilidade }\left(E_{1}^{(1)}\right)+\text { probabilidade }\left(E_{1}^{(2)}\right) \\
& =p_{11}+p_{12}
\end{aligned}
$$

2. Para esta modelagem é considerado $\mu_{2}>0$, uma vez que $\mu_{2}=0$ implicaria em 'deadlock' do sistema nos estados $E_{1}^{(2)}$ e $E_{2}$.

Temos então as seguintes equações de equilíbrio:

Equação para o estado $E_{0}$ :
$\mathrm{A}: \quad-\lambda p_{0}$
$+\mu_{1} p_{11}$
$+\mu_{2} p_{12}$
$=0$

Equações para o estado $E_{1}$ :
B: $\lambda p_{0} \quad-\left(\lambda+\mu_{1}\right) p_{11}$
$+\mu_{2} p_{2}=0$
C:
$-\left(\lambda+\mu_{2}\right) p_{12}+\mu_{1} p_{2}=0$

Equação para o estado $E_{\mathfrak{q}}$ :

D: $\quad \begin{array}{lll}\lambda p_{i-1} & -(\lambda+\mu) p_{i} & +\mu p_{i+1}\end{array}$

E passando à resolução do sistema de equações acima, temos

Da equação $C$ vem

$$
p_{12}=\frac{\mu_{1}}{\lambda+\mu_{2}} p_{2}
$$


Somando as equações $A$ e $B$, temos

$$
\begin{gathered}
-\lambda p_{11}+\mu_{2} p_{12}+\mu_{2} p_{2}=0 \\
-\lambda p_{11}+\mu_{2} \frac{\mu_{1}}{\lambda+\mu_{2}} p_{2}+\mu_{2} p_{2}=0 . \\
p_{11}=\frac{\mu_{2}}{\lambda}\left(1+\frac{\mu_{1}}{\lambda+\mu_{2}}\right) p_{2} \\
p_{1}=p_{11}+p_{12} \\
=p_{2}\left[\frac{\mu_{1}}{\lambda+\mu_{2}}+\frac{\mu_{2}}{\lambda}\left(1+\frac{\mu_{1}}{\lambda+\mu_{2}}\right)\right] \\
=p_{2}\left[\frac{\lambda \mu_{1}+\mu_{2}\left(\lambda+\mu_{2}\right)+\mu_{1} \mu_{2}}{\lambda\left(\lambda+\mu_{2}\right)}\right]=p_{2}\left[\frac{\left(\lambda+\mu_{2}\right) \mu_{1}+\left(\lambda+\mu_{2}\right) \mu_{2}}{\lambda\left(\lambda+\mu_{2}\right)}\right] \\
=\frac{\mu}{\lambda} p_{2}
\end{gathered}
$$

Da equação $A$ vem

$$
\begin{aligned}
p_{o} & =\frac{1}{\lambda}\left[\mu_{1} \frac{\mu_{2}}{\lambda}\left(1+\frac{\mu_{1}}{\lambda+\mu_{2}}\right)+\mu_{2} \frac{\mu_{1}}{\lambda+\mu_{2}}\right] p_{2} \\
& =\frac{\mu_{1} \mu_{2}(2 \lambda+\mu)}{\lambda^{2}\left(\lambda+\mu_{2}\right)} p_{2}
\end{aligned}
$$

Para os estados $i \geq 3$ temos

A equação $D$ para $i=2$

$$
\begin{aligned}
& \lambda p_{1}-(\lambda+\mu) p_{2}+\mu p_{3}=0 \\
& \lambda \frac{\mu}{\lambda} p_{2}-\left(\lambda+\mu_{2}\right) p_{2}+\mu p_{3}=0 \\
& p_{3}=\frac{\lambda}{\mu} p_{2}
\end{aligned}
$$

A equação $D$ para $i=3$

$$
\begin{aligned}
& \lambda p_{2}-(\lambda+\mu) p_{3}+\mu p_{4}=0 \\
& \lambda p_{2}-(\lambda+\mu) \frac{\lambda}{\mu} p_{2}+\mu p_{4}=0 \\
& p_{4}=\left(\frac{\lambda}{\mu}\right)^{2} p_{2}
\end{aligned}
$$

E genericamente

$$
p_{i}=\left(\frac{\lambda}{\mu}\right)^{i-2} p_{2}, \quad i \geq 3
$$


Temos também que

$$
\begin{aligned}
& \sum_{i=1}^{\infty} p_{i}=1 \\
& {\left[\frac{\mu_{1} \mu_{2}(2 \lambda+\mu)}{\lambda^{2}\left(\lambda+\mu_{2}\right)}+\frac{\mu}{\lambda}+1+\sum_{i=1}^{\infty}\left(\frac{\lambda}{\mu}\right)^{i}\right] p_{2}=1}
\end{aligned}
$$

Mas

$$
\sum_{i=1}^{\infty}\left(\frac{\lambda}{\mu}\right)^{i}=\frac{\frac{\lambda}{\mu}}{1-\frac{\lambda}{\mu}}=\frac{\lambda}{\mu-\lambda}
$$

Portanto

$$
\begin{aligned}
& {\left[\frac{\mu_{1} \mu_{2}(2 \lambda+\mu)}{\lambda^{2}\left(\lambda+\mu_{2}\right)}+\frac{\mu(\mu-\lambda)+\lambda(\mu-\lambda)+\lambda^{2}}{\lambda(\mu-\lambda)}\right] p_{2}=1 } \\
\Rightarrow & p_{2}=\frac{\lambda^{2}\left(\lambda+\mu_{2}\right)(\mu-\lambda)}{\mu_{1} \mu_{2}(2 \lambda+\mu)(\mu-\lambda)+\mu^{2} \lambda\left(\lambda+\mu_{2}\right)}
\end{aligned}
$$

\subsection{1 - Análise para tempo no sistema}

Seja $N_{s}^{2}$ - número médio de jobs no sistema de dois servidores

$$
\begin{aligned}
N_{s}^{2} & =\sum_{i=0}^{\infty} i p_{i} \\
& =p_{1}+2 p_{2}+\sum_{i=3}^{\infty} i\left(\frac{\lambda}{\mu}\right)^{i-2} p_{2} \\
& =\left[\frac{\mu}{\lambda}+2+\sum_{i=1}^{\infty}(i+2)\left(\frac{\lambda}{\mu}\right)^{i}\right] p_{2} \\
& =\left[\frac{\mu}{\lambda}+2+2 \sum_{i=1}^{\infty}\left(\frac{\lambda}{\mu}\right)^{i}+\sum_{i=1}^{\infty} i\left(\frac{\lambda}{\mu}\right)^{i}\right] p_{2}
\end{aligned}
$$

Seja então $A=\frac{\lambda}{\mu}$

$$
\begin{aligned}
\frac{1}{A}+2+2 \frac{A}{1-A}+\frac{A}{(1-A)^{2}} & =\frac{(1-A)^{2}+2 A(1-A)^{2}+2 A^{2}(1-A)+A^{2}}{A(1-A)^{2}} \\
& =\frac{(1-A)^{2}+A^{2}+2 A(1-A)[1-A+A]}{A(1-A)^{2}} \\
& =\frac{(1-A+A)^{2}}{A(1-A)^{2}}=\frac{1}{A(1-A)^{2}}
\end{aligned}
$$


Logo

$$
\frac{1}{A(1-A)^{2}}=\frac{1}{\frac{\lambda}{\mu}\left(1-\frac{\lambda}{\mu}\right)^{2}}=\frac{1}{\frac{\lambda}{\mu}\left(\frac{\mu-\lambda}{\mu}\right)^{2}}=\frac{\mu^{3}}{\lambda(\mu-\lambda)^{2}}
$$

Portanto

$$
\begin{aligned}
N_{s}^{2} & =\frac{\mu^{3}}{\lambda(\mu-\lambda)^{2}} \frac{\lambda^{2}\left(\lambda+\mu_{2}\right)(\mu-\lambda)}{\mu_{1} \mu_{2}(2 \lambda+\mu)(\mu-\lambda)+\mu^{2} \lambda\left(\lambda+\mu_{2}\right)} \\
& =\frac{\lambda \mu^{3}\left(\lambda+\mu_{2}\right)}{(\mu-\lambda)\left[\mu_{2} \mu_{2}(2 \lambda+\mu)(\mu-\lambda)+\mu^{2} \lambda\left(\lambda+\mu_{2}\right)\right]}
\end{aligned}
$$

$\mathrm{E}$ por Little obtemos o tempo médio no sistema com dois servidores

$$
T_{s}^{2}=\frac{\mu^{3}\left(\lambda+\mu_{2}\right)}{(\mu-\lambda)\left[\mu_{1} \mu_{2}(2 \lambda+\mu)(\mu-\lambda)+\mu^{2} \lambda\left(\lambda+\mu_{2}\right)\right]}
$$

É fácil verificar que para $\mu_{1}=\mu_{2}$ a fórmula acima fornece a conhecida expressão para cálculo de tempo médio no sistema. $M / M / 2$ com servidores iguais (vide [16],[30]).

$$
\begin{aligned}
& \left.T_{s}^{2}\right|_{\mu_{1}=\mu_{2}=\frac{\mu}{2}}=\frac{\mu^{3}\left(\lambda+\frac{\mu}{2}\right)}{(\mu-\lambda)\left[\frac{\mu^{2}}{4}(2 \lambda+\mu)(\mu-\lambda)+\mu^{2}\left(\lambda+\frac{\mu}{2}\right)\right]} \\
& =\frac{\frac{\mu^{3}}{2}(2 \lambda+\mu)}{(\mu-\lambda)\left[\frac{\mu^{2}}{4}(2 \lambda+\mu)(\mu-\lambda)+\mu^{2} \lambda(2 \lambda+\mu) \frac{1}{2}\right]}=\frac{2 \mu}{(\mu-\lambda)[(\mu-\lambda)+2 \lambda]} \\
& =\frac{2 \mu}{\left(\mu^{2}-\lambda^{2}\right)}=\frac{1}{\frac{\mu}{2}\left(1-\rho^{2}\right)}
\end{aligned}
$$

É importante notar que

$$
\lim _{\mu_{2} \rightarrow 0} T_{s}^{2}=\left.T_{s}^{2}\right|_{\mu_{2}=0} \neq T_{s}^{1}
$$

ou seja, a fórmula obtida para $T_{s}^{2}$ só é válida para dois servidores ativos. Isto ocorre devido à modelagem ter sido feita supondo $\mu_{2}>0$ e $\mu_{1} \geq \mu_{2}$.

\section{Generalizações do modelo apresentado}

O modelo apresentado para o sistema $S(\lambda, \mu, 1,2)$ através do diagrama de transição de estados poderia ter as seguintes generalizações:

1. Poderia se considerar o sistema $S(\lambda, \mu, 1,2)$ conforme [23], onde valem as mesmas especificações aqui apresentadas para o modelo, com a diferença que quando um job 
chega no sistema e encontra os dois servidores ocupados, ele se junta à fila de espera com probabilidade $\beta(0 \leq \beta \leq 1)$ e com probabilidade $(1-\beta)$ ele é impedido de se juntar ao sistema. O sistema assim caracterizado é chamado sistema com 'balking'. Para este sistema o diagrama de transição de estados seria

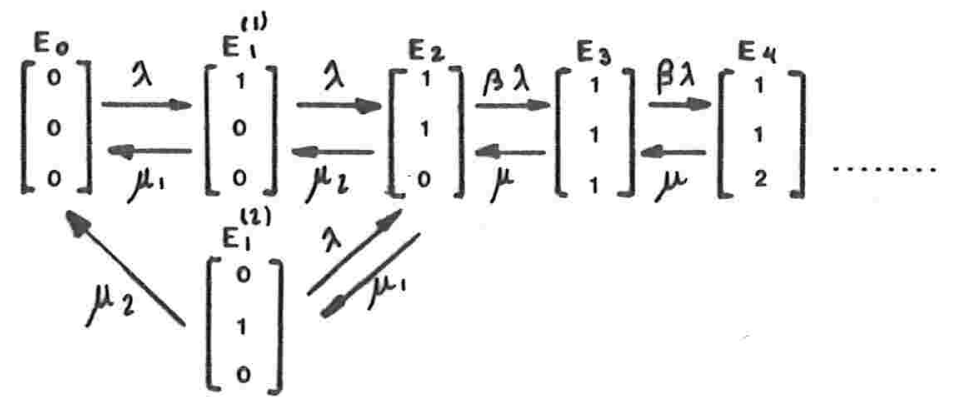

Logo, apenas as equações dos estados $E_{i}, i \geq 2$, sofreriam alterações em relação às do modelo inicial, resultando em:

Equação para o estado $E_{2}: \quad \lambda p_{1}+\mu p_{3}-(\beta \lambda+\mu) p_{2}=0$

$$
\Rightarrow p_{2}=\beta \frac{\lambda}{\mu} p_{2}
$$

Equação para o estado $E_{i}, \quad i \geq 3: \quad(\beta \lambda) p_{i-1}+\mu p_{i+1}-(\beta \lambda+\mu) p_{i}=0$

$$
\Rightarrow p_{i}=\left(\beta \frac{\lambda}{\mu}\right)^{i-1}
$$

Analogamente ao modelo inicial,

$$
\begin{aligned}
& {\left[\frac{\mu_{1} \mu_{2}(2 \lambda+\mu)}{\lambda^{2}\left(\lambda+\mu_{2}\right)}+\frac{\mu}{\lambda}+1+\sum_{i=1}^{\infty}\left(\beta \frac{\lambda}{\mu}\right)^{i}\right] p_{2}=1 } \\
\Longleftrightarrow & {\left[\frac{\mu_{1} \mu_{2}(\lambda+\mu)}{\lambda^{2}\left(\lambda+\mu_{2}\right)}+\frac{\mu(1+\rho(1-\beta))}{\lambda(1-\beta \rho)}\right] p_{2}=1 } \\
\Longleftrightarrow & p_{2}=\frac{\lambda^{2}\left(\lambda+\mu_{2}\right)(1-\beta \rho)}{\mu_{1} \mu_{2}(2 \lambda+\mu)(1-\beta \rho)+\mu(1+\rho(1-\beta)) \lambda\left(\lambda+\mu_{2}\right)}
\end{aligned}
$$


E ainda,

$$
\begin{aligned}
N_{s} & =\sum_{i=0}^{\infty} i p_{i} \\
& =\left[\frac{1}{\rho}+2+2 \sum_{i=0}^{\infty}(\beta \rho)^{i}+\sum_{i=1}^{\infty} i(\beta \rho)^{i}\right] p_{2} \\
& =\left[\frac{1}{\rho}+2+\frac{2(\beta \rho)}{1-\beta \rho}+\frac{\beta \rho}{(1-\beta \rho)^{2}}\right] p_{2} \\
& =\left[\frac{(1-\beta \rho)^{2}+2 \rho(1-\beta \rho)^{2}+2(\beta \rho) \rho(1-\beta \rho)+\beta \rho^{2}}{\rho(1-\beta \rho)^{2}}\right] p_{2} \\
& =\left[\frac{1+\rho(1-\beta)(2-\beta \rho)}{\rho(1-\beta \rho)^{2}}\right] p_{2}
\end{aligned}
$$

E resultaria a seguinte expressão para tempo médio no sistema com 'balking'

$$
T_{s}^{b}=\frac{(1+\rho(1-\beta)(2-\beta \rho)) \mu\left(\lambda+\mu_{2}\right)}{(1-\beta \rho)\left[\mu_{1} \mu_{2}(2 \lambda+\mu)(1-\beta \rho)+\mu(1+\rho(1-\beta)) \lambda\left(\lambda+\mu_{2}\right)\right]}
$$

Nós nos deteremos ao caso $\beta=1$, onde o job nunca é impedido de se juntar ao sistema, o que é viável, uma vez que consideramos uma fila com capacidade infinita.

2. Poderia se considerar o sistema com a seguinte generalização apresentada em [20]: um job que chega no sistema e encontra os dois servidores livres será atendido pelo servidor 1 com probabilidade $\pi_{1}$ e pelo servidor 2 com probabilidade $\pi_{2}$, onde $\pi_{1}+\pi_{2}=1$.

Desta forma teríamos:

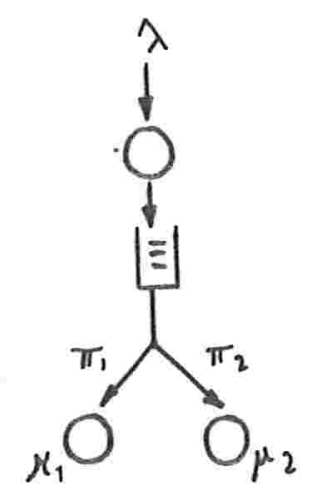




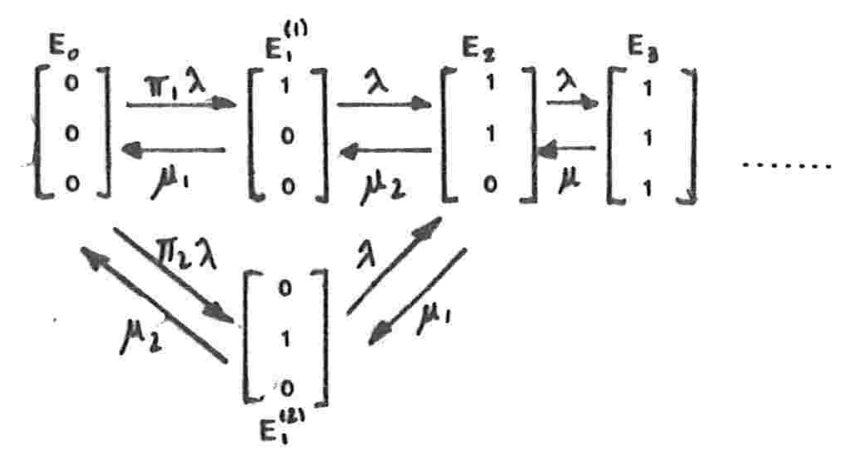

$\mathrm{E}$ as equações do sistema em equilíbrio seriam:

$$
\begin{array}{lllllll}
X: & -\lambda p_{o} & +\mu_{1} p_{11} & +\mu_{2} p_{12} & & =0 & \\
Y: & \pi_{1} \lambda p_{o} & -\left(\lambda+\mu_{1}\right) p_{11} & & +\mu_{2} p_{2} & =0 & \\
Z: & \pi_{2} \lambda p_{o} & & -\left(\lambda+\mu_{2}\right) p_{12} & +\mu_{1} p_{2} & & =0 \\
W: & \lambda p_{11} & +\lambda p_{12} & -(\lambda+\mu) p_{2} & +\mu p_{3} & =0 & \\
W: & \lambda p_{i-1} & -(\lambda+\mu) p_{i} & +\mu p_{i+1} . & & =0, & i \geq 3
\end{array}
$$

E obteríamos:

Somando as equações $X, Y$ e $Z$, temos: $\quad-\lambda p_{11}-\lambda p_{12}+\left(\mu_{1}+\mu_{2}\right) p_{2}=0$

$$
\Rightarrow p_{1}=\frac{\mu}{\lambda} p_{2}
$$

Da equação $W$ vem: $\quad \lambda p_{1}-(\lambda+\mu) p_{2}+\mu p_{3}=0$

$$
\Rightarrow p_{3}=\frac{\lambda}{\mu} p_{2}
$$

$\pi_{1} *$ equação $X+$ equação $Y=0 \Rightarrow-\left(\lambda+\pi_{2} \mu_{1}\right) p_{11}+\pi_{1} \mu_{2} p_{12}+\mu_{2} p_{2}=0$.

$$
\begin{aligned}
\text { Mas } & p_{2}=\rho p_{1}=\rho\left(p_{11}+p_{12}\right)=\rho p_{11}+\rho p_{12} \\
\Rightarrow & \left(-\left(\lambda+\pi_{2} \mu_{1}\right)+\mu_{2} \rho\right) p_{11}+\left(\pi_{1} \mu_{2}+\mu_{2} \rho\right) p_{12}=0 \\
& \Rightarrow p_{11}=\left[\frac{\pi_{1} \mu_{2}+\mu_{2} \rho}{\left(\lambda+\pi_{2} \mu_{1}\right)-\mu_{2} \rho}\right] p_{12} \\
\text { Mas } & p_{11}+p_{12}=p_{1} \\
\Rightarrow & \left(\frac{\pi_{1} \mu_{2}+\mu_{2} \rho}{\lambda+\pi_{2} \mu_{1}-\mu_{2} \rho}+1\right) p_{12}=p_{1} \\
& \Rightarrow\left(\frac{\pi_{1} \mu_{2}+\mu_{2} \rho+\lambda+\pi_{2} \mu_{1}-\mu_{2} \rho}{\lambda+\pi_{2} \mu_{1}-\mu_{2} \rho}\right) p_{12}=p_{1}
\end{aligned}
$$




$$
\begin{aligned}
& \Rightarrow p_{12}=\left(\frac{\lambda+\pi_{2} \mu_{1}-\mu_{2} \rho}{\lambda+\pi_{2} \mu_{1}+\pi_{1} \mu_{2}}\right) p_{1} \\
& \Rightarrow p_{11}=p_{1}-p_{12}=p_{1}-\left(\frac{\lambda+\pi_{2} \mu_{1}-\mu_{2} \rho}{\lambda+\pi_{2} \mu_{1}+\pi_{1} \mu_{2}}\right) p_{1} \\
& \Rightarrow p_{11}=\left[\frac{\left(\pi_{1}+\rho\right) \mu_{2}}{\lambda+\pi_{2} \mu_{1}+\pi_{1} \mu_{2}}\right] p_{1}
\end{aligned}
$$

Substituindo (1) e (2) em $X$

$$
\begin{aligned}
& -\lambda p_{o}+\mu_{1}\left[\frac{\left(\pi_{1}+\rho\right) \mu_{2}}{\lambda+\pi_{2} \mu_{1}+\pi_{1} \mu_{2}}\right] p_{1}+\mu_{2}\left[\frac{\lambda+\pi_{2} \mu_{1}-\mu_{2} \rho}{\lambda+\pi_{2} \mu_{1}+\pi_{1} \mu_{2}}\right] p_{1}=0 \\
\Longleftrightarrow & -\lambda p_{o}+p_{1}\left[\frac{\mu_{1} \mu_{2}\left(\pi_{1}+\rho\right)+\mu_{2}\left(\lambda+\pi_{2} \mu_{1}-\mu_{2} \rho\right)}{\lambda+\pi_{2} \mu_{1}+\pi_{1} \mu_{2}}\right]=0 \\
\Longleftrightarrow & -\lambda p_{o}+p_{1}\left[\frac{\mu_{1} \mu_{2}(1+\rho)+\mu_{2}\left(\lambda-\mu_{2} \rho\right)}{\lambda+\pi_{2} \mu_{1}+\pi_{1} \mu_{2}}\right]=0 \\
\Longleftrightarrow & p_{o}=\left[\frac{\mu_{1} \mu_{2}(1+\rho)+\mu_{2}\left(\lambda-\mu_{2} \rho\right)}{\lambda\left(\lambda+\pi_{2} \mu_{1}+\pi_{1} \mu_{2}\right)}\right] p_{1}
\end{aligned}
$$

Ou ainda

$$
\begin{aligned}
& p_{o}=\left[\frac{\mu_{1} \mu_{2}(1+\rho)+\mu_{2}\left(\lambda-\mu_{2} \rho\right)}{\lambda\left(\lambda+\pi_{2} \mu_{1}+\pi_{1} \mu_{2}\right)}\right] \frac{\mu}{\lambda} p_{2} \\
& {\left[\mu_{1} \mu_{2}(1+\rho)+\mu_{2}\left(\lambda-\mu_{2} \rho\right)\right] \mu=\mu_{1} \mu_{2}(\mu+\lambda)+\mu_{2} \lambda \mu-\mu_{2}^{2} \lambda=\mu_{1} \mu_{2}(\mu+2 \lambda)}
\end{aligned}
$$

Portanto

$$
p_{o}=\left[\frac{\mu_{1} \mu_{2}(\mu+2 \lambda)}{\lambda^{2}\left(\lambda+\pi_{1} \mu_{2}+\pi_{2} \mu_{1}\right)}\right] p_{2}
$$

$\mathrm{E}$ analogamente ao modelo sem generalização

$$
\begin{aligned}
p_{2} & =\left[\frac{\mu_{1} \mu_{2}(\mu+2 \lambda)}{\lambda^{2}\left(\lambda+\pi_{2} \mu_{1}+\pi_{1} \mu_{2}\right)}+\frac{\mu^{2}}{\lambda(\mu-\lambda)}\right]^{-1} \\
& =\frac{\lambda^{2}\left(\lambda+\pi_{2} \mu_{1}+\pi_{1} \mu_{2}\right)(\mu-\lambda)}{\mu_{1} \mu_{2}(\mu+2 \lambda)(\mu-\lambda)+\mu^{2} \lambda\left(\lambda+\pi_{2} \mu_{1}+\pi_{1} \mu_{2}\right)} \\
\text { e } N_{s} & =\frac{\mu^{3}}{\lambda(\mu-\lambda)^{2}} p_{2}
\end{aligned}
$$

E resultaria a seguinte expressão para tempo médio no sistema generalizado:

$$
T_{s}^{g}=\frac{\mu^{3}\left(\lambda+\pi_{2} \mu_{1}+\pi_{1} \mu_{2}\right)}{(\mu-\lambda)\left[\mu_{1} \mu_{2}(\mu+2 \lambda)(\mu-\lambda)+\mu^{2} \lambda\left(\lambda+\pi_{2} \mu_{1}+\pi_{1} \mu_{2}\right)\right]}
$$

FATO 4.1: $T_{s}^{g}$ é minimizado $\operatorname{com} \pi_{1}=1$ e $\pi_{2}=0$. 


\section{DEMONSTRAÇÃO:}

Basta mostrar que

$$
\begin{gathered}
\frac{\mu^{3}\left(\lambda+\pi_{2} \mu_{1}+\pi_{1} \mu_{2}\right)}{(\mu-\lambda)\left[\mu_{1} \mu_{2}(\mu+2 \lambda)(\mu-\lambda)+\mu^{2} \lambda\left(\lambda+\pi_{2} \mu_{1}+\pi_{1} \mu_{2}\right)\right]} \\
\geq \frac{\mu^{3}\left(\lambda+\mu_{2}\right)}{(\mu-\lambda)\left[\mu_{1} \mu_{2}(\mu+2 \lambda)(\mu-\lambda)+\mu^{2} \lambda\left(\lambda+\mu_{2}\right)\right]} \\
\forall\left(\pi_{1}, \pi_{2}\right) \in V=\left\{\left(\pi_{1}, \pi_{2}\right) \in R_{+} \times R_{+}: \pi_{1}+\pi_{2}=1\right\} \\
\Longleftrightarrow\left(\lambda+\pi_{2} \mu_{1}+\pi_{1} \mu_{2}\right)\left[\mu_{1} \mu_{2}(\mu+2 \lambda)(\mu-\lambda)+\mu^{2} \lambda\left(\lambda+\mu_{2}\right)\right] \\
\geq\left(\lambda+\mu_{2}\right)\left[\mu_{1} \mu_{2}(\mu+2 \lambda)(\mu-\lambda)+\mu^{2} \lambda\left(\lambda+\pi_{2} \mu_{1}+\pi_{1} \mu_{2}\right)\right] \quad \forall\left(\pi_{1}, \pi_{2}\right) \in V \\
\Longleftrightarrow\left(\lambda+\pi_{2} \mu_{1}+\pi_{1} \mu_{2}\right) \mu_{1} \mu_{2}(\mu+2 \lambda)(\mu-\lambda) \\
\geq\left(\lambda+\mu_{2}\right) \mu_{1} \mu_{2}(\mu+2 \lambda)(\mu-\lambda) \quad \forall\left(\pi_{1}, \pi_{2}\right) \in V \\
\Longleftrightarrow \pi_{2} \mu_{1}+\pi_{1} \mu_{2} \geq \mu_{2} \quad \forall\left(\pi_{1}, \pi_{2}\right) \in V \\
\Leftrightarrow \pi_{2} \mu_{1} \geq\left(1-\pi_{1}\right) \mu_{2}=\pi_{2} \mu_{2} \quad \forall\left(\pi_{1}, \pi_{2}\right) \in V \\
\mu_{1} \geq \mu_{2} \quad
\end{gathered}
$$

Como $\mu_{1} \geq \mu_{2}$ por hipótese, o fato está demonstrado.

Este fato mostra que quando um job chega no sistema e encontra os dois servidores livres, o servidor mais forte é a melhor escolha, o que vem de acordo com a intuição.

Logo, não há perda de generalidade em utilizarmos o modelo inicialmente apresentado.

Assim, uma vez obtida a expressão para $T_{s}^{2}$, temos interesse em obter a distribuição da capacidade total de atendimento entre os servidores que minimiza o tempo médio no sistema. Para isso basta determinar a solução $\left(\mu_{1}^{*}, \mu_{2}^{*}\right)$ do problema $P$ abaixo definido, sendo

$\mu_{i}^{*}$ - a capacidade de atendimento ótima do servidor $i$, em relação a tempo médio no sistema, $i=1,2$.

$$
\begin{aligned}
P: \quad \min & T_{s}^{2}\left(\mu_{1}, \mu_{2}\right) \\
\text { sujeito a } & \mu_{1}+\mu_{2}=\mu \\
& \mu_{1} \geq \mu_{2} \\
& \mu_{2}>0
\end{aligned}
$$

FATO 4.2: $T_{s}^{2}\left(\mu_{1}, \mu_{2}\right)$ é contínua em $A=\left\{\left(\mu_{1}, \mu_{2}\right) \in R_{+} \times R_{+}^{*} \mid \mu_{1}+\mu_{2}=\mu\right\}$. 
DEMONSTRAÇÃO:

$$
\begin{aligned}
T_{s}^{2}\left(\mu_{1}, \mu_{2}\right) & =\frac{\mu^{3}\left(\lambda+\mu_{2}\right)}{(\mu-\lambda)\left[\mu_{1} \mu_{2}(2 \lambda+\mu)(\mu-\lambda)+\mu^{2} \lambda\left(\lambda+\mu_{2}\right)\right]} \\
& =\frac{N\left(\mu_{1}, \mu_{2}\right)}{D\left(\mu_{1}, \mu_{2}\right)}
\end{aligned}
$$

$N\left(\mu_{1}, \mu_{2}\right)$ é produto de funções contínuas em $A$, logo é contínua em $A$.

$D\left(\mu_{1}, \mu_{2}\right)=\mu_{1} \mu_{2}(2 \lambda+\mu)(\mu-\lambda)^{2}+\left(\lambda+\mu_{2}\right) \mu^{2} \lambda(\mu-\lambda)$.

$D$ é soma de funções contínuas em $A$, logo é contínua.

E ainda, $D\left(\mu_{1}, \mu_{2}\right)>0$ pois $M>\lambda>0$ e $\mu_{2}>0$.

Portanto temos $T_{s}^{2}$ contínua em $A$.

Seja o problema relaxado $P_{r}$

$$
\begin{aligned}
P_{r}: \quad \min & T_{s}^{2}\left(\mu_{1}, \mu_{2}\right) \\
\text { sujeito a } & \mu_{1}+\mu_{2}=\mu \\
& \mu_{1}>0 \\
& \mu_{2}>0
\end{aligned}
$$

FATO 4.3: A solução de $P_{r}$ é dada por $\left(\mu_{1}^{*}, \mu_{2}^{*}\right)$, com

$$
\begin{aligned}
& \mu_{2}^{*}=-\lambda+\sqrt{\lambda} \sqrt{\lambda+\mu}=\lambda\left(\sqrt{1+\frac{1}{\rho}}-1\right) \\
& \mu_{1}^{*}=\mu-\mu_{2}^{*}
\end{aligned}
$$

\section{DEMONSTRAÇÃO:}

Seja $G\left(\mu_{2}\right)=T_{s}^{2}\left(\mu-\mu_{2}, \mu_{2}\right)$.

O conjunto dos pontos viáveis de $P_{r}$ pode ser escrito na forma

$$
B=\left\{\mu_{2} \in\right] 0, \mu\left[\mid \mu-\mu_{2} \in\right] 0, \mu[\}
$$

$G$ é contínua em $B$, pois pelo Fato $4.2, T_{s}^{2}$ é contínua em $A \supset B$.

$$
\begin{aligned}
G\left(\mu_{2}\right) & =\frac{\mu^{3}\left(\lambda+\mu_{2}\right)}{(\mu-\lambda)\left[\left(\mu-\mu_{2}\right) \mu_{2}(2 \lambda+\mu)(\mu-\lambda)+\mu^{2} \lambda\left(\lambda+\mu_{2}\right)\right]} \\
G^{\prime}\left(\mu_{2}\right) & =\frac{\mu^{3}(\mu-\lambda) C-\mu^{3}\left(\lambda+\mu_{2}\right)\left[(\mu-\lambda)^{2}(2 \lambda+\mu)\left(\mu-2 \mu_{2}\right)+\mu^{2} \lambda(\mu-\lambda)\right]}{(\mu-\lambda)^{2} C^{2}}
\end{aligned}
$$

onde $C=\left(\mu-\mu_{2}\right) \mu_{2}(2 \lambda+\mu)(\mu-\lambda)+\mu^{2} \lambda\left(\lambda+\mu_{2}\right)$ 
Podemos então escrever

$$
\begin{aligned}
& G^{\prime}\left(\mu_{2}\right)=\frac{P\left(\mu_{2}\right)}{Q\left(\mu_{2}\right)} \\
& Q\left(\mu_{2}\right)=(\mu-\lambda)^{2} C^{2}>0 \text { pois } \quad \mu>\lambda>0 \quad \text { e } \quad \mu_{2}>0 .
\end{aligned}
$$

Se $\mu_{2}^{*}$ é mínimo de $G$ então $G^{\prime}\left(\mu_{2}^{*}\right)=0$, assim vamos calcular os pontos que anulam $G^{\prime}$, ou equivalentemente, os pontos que anulam $P$.

$$
\begin{aligned}
& P\left(\mu_{2}\right)=0 \\
\Longleftrightarrow & (\mu-\lambda)^{2}\left(\mu-\mu_{2}\right) \mu_{2}(2 \lambda+\mu)+(\mu-\lambda) \mu^{2} \lambda\left(\lambda+\mu_{2}\right)- \\
& -\left(\lambda+\mu_{2}\right)(\mu-\lambda)^{2}(2 \lambda+\mu)\left(\mu-2 \mu_{2}\right)-\left(\lambda+\mu_{2}\right) \mu^{2} \lambda(\mu-\lambda)=0 \\
\Longleftrightarrow & \left(\mu-\mu_{2}\right) \mu_{2}-\left(\lambda+\mu_{2}\right)\left(\mu-2 \mu_{2}\right)=0 \\
\Longleftrightarrow & \mu_{2}^{2}+2 \lambda \mu_{2}-\lambda \mu=0
\end{aligned}
$$

As raízes desse polinômio de $2^{\circ}$ grau são dadas por:

$\mu_{2}^{\prime}=-\lambda-\sqrt{\lambda} \sqrt{\lambda+\mu}<0 \mathrm{e}$

$\mu_{2}^{\prime \prime}=-\lambda+\sqrt{\lambda} \sqrt{\lambda+\mu}$

Obviamente $\mu_{2}^{\prime}$ não é de nosso interesse pois $\mu_{2}^{\prime} \notin B$.

Vamos mostrar que $\mu_{2}^{*}=\mu_{2}^{\prime \prime}=-\lambda+\sqrt{\lambda} \sqrt{\lambda+\mu}$ é solução de $P_{r}$.

i) $\mu_{2}^{*} \in B$

$$
\begin{aligned}
& \mu_{2}^{*}=-\lambda+\sqrt{\lambda} \sqrt{\lambda+\mu}=\sqrt{\lambda}(-\sqrt{\lambda}+\sqrt{\lambda+\mu})>0 \text { pois } \mu>0 \\
& \mu_{2}^{*}<\mu \Longleftrightarrow-\lambda+\sqrt{\lambda} \sqrt{\lambda+\mu}<\mu \\
& \Longleftrightarrow \sqrt{\lambda}<\sqrt{\lambda+\mu}
\end{aligned}
$$

Como $\mu>0$ temos $\mu_{2}^{*}<\mu$

$\mu-\mu_{2}^{*}=\mu+\lambda-\sqrt{\lambda} \sqrt{\lambda+\mu}=\sqrt{\mu+\lambda}(\sqrt{\lambda+\mu}-\sqrt{\lambda})>0$

$\mu-\mu_{2}^{*}<\mu$ pois $\mu_{2}^{*}>0$

ii) $\mu_{2}^{*}$ é ponto de mínimo para $G$.

Basta estudar o sinal de $G^{\prime}$, ou equivalentemente, estudar o sinal de $P$.

Mas temos pelo estudo do polinômio dado em (1)

$$
\begin{array}{llll}
G^{\prime}\left(\mu_{2}^{*}\right)=0 & & \\
G^{\prime}\left(\mu_{2}\right)<0 & \text { para } & 0<\mu_{2}<\mu_{2}^{*} \\
G^{\prime}\left(\mu_{2}\right)>0 & \text { para } & \mu_{2}^{*}<\mu_{2}<\mu
\end{array}
$$


Logo, a função $G$ é decrescente no intervalo $] 0, \mu_{2}^{*}$ [ e é crescente no intervalo ] $\mu_{2}^{*}, \mu[\mathrm{e}$ portanto $\mu_{2}^{*}$ é ponto de mínimo para $G$.

Temos então, por i) e ii), que $\left(\mu_{1}^{*}, \mu_{2}^{*}\right)$ é solução de $P_{r}$ onde

$$
\mu_{2}^{*}=-\lambda+\sqrt{\lambda} \sqrt{\lambda+\mu}=\lambda\left(\sqrt{1+\frac{1}{\rho}}-1\right) \text { e } \mu_{1}^{*}=\mu-\mu_{2}^{*} .
$$

FATO 4.4: A solução de $P$ é dada pela solução de $P_{r}$.

\section{DEMONSTRAÇÃO:}

Seja $\left(\mu_{1}^{*}, \mu_{2}^{*}\right)$ a solução de $P_{r}$, que é dada por

$$
\begin{aligned}
& \mu_{1}^{*}=\mu-\mu_{2}^{*} \\
& \mu_{2}^{*}=-\lambda+\sqrt{\lambda} \sqrt{\lambda+\mu}
\end{aligned}
$$

i) $\left(\mu_{1}^{*}, \mu_{2}^{*}\right)$ é viável em $P$.

Como $\left(\mu_{1}^{*}, \mu_{2}^{*}\right)$ é viável em $P_{r}$, resta apenas provar que $\mu_{1}^{*} \geq \mu_{2}^{*}$. Mas

$$
\begin{aligned}
\mu_{1}^{*} \geq \mu_{2}^{*} & \Longleftrightarrow \lambda+\mu-\sqrt{\lambda} \sqrt{\lambda+\mu} \geq-\lambda+\sqrt{\lambda} \sqrt{\lambda+\mu} \\
& \Longleftrightarrow 2 \lambda+\mu \geq 2 \sqrt{\lambda} \sqrt{\lambda+\mu} \\
& \Longleftrightarrow 4 \lambda^{2}+4 \lambda \mu+\mu^{2} \geq 4 \lambda(\lambda+\mu) \\
& \Longleftrightarrow \mu^{2} \geq 0
\end{aligned}
$$

Como $\mu>0$ temos que $\mu_{1}^{*}>\mu_{2}^{*}$.

ii) $\left(\mu_{1}^{*}, \mu_{2}^{*}\right)$ é solução de $P$.

Seja $V r$ o conjunto dos pontos viáveis de $P_{r}$ e $V$ o conjunto dos pontos viáveis de $P$.

$$
\begin{array}{rll} 
& T_{s}^{2}\left(\mu_{1}^{*}, \mu_{2}^{*}\right) \leq T_{s}^{2}\left(\mu_{1}, \mu_{2}\right) \quad \forall\left(\mu_{1}, \mu_{2}\right) \in V r \quad \text { (pois }\left(\mu_{1}^{*}, \mu_{2}^{*}\right) \text { é solução de } P_{r} \text { ) } \\
\Leftrightarrow T_{s}^{2}\left(\mu_{1}^{*}, \mu_{2}^{*}\right) \leq T_{s}^{2}\left(\mu_{1}, \mu_{2}\right) \quad \forall\left(\mu_{1}, \mu_{2}\right) \in V \quad & \text { (pois } V \subset V r)
\end{array}
$$

Logo, por i), temos que $\left(\mu_{1}^{*}, \mu_{2}^{*}\right)$ é solução de $P$.

A solução de $P$ descreve, na verdade, a distribuição ótima de capacidades entre os servidores de um sistema $S(\lambda, \mu, 1,2)$. É fato que essa distribuição minimiza não somente o tempo médio no sistema $S(\lambda, \mu, 1,2)$, mas também o número médio de jobs no sistema. 
COROLÁRIO 4.5: $\left(\frac{\mu}{2}, \frac{\mu}{2}\right)$ nunca é solução de $P$.

\section{DEMONSTRAÇÃO:}

É óbvia, pois na demonstração do fato 4.4 vimos que se $\left(\mu_{1}^{*}, \mu_{2}^{*}\right)$ é solução de $P$, e $\mu>0$ temos que $\mu_{1}^{*}>\mu_{2}^{*}$.

Este corolário garante que o sistema $S(\lambda, \mu, 1,2)$ homogêneo nunca é ótimo.

A seguir mostraremos que o sistema de dois servidores tem desempenho pior que o sistema de um servidor correspondente e também um resultado bastante interessante sobre a comparação de sistemas de dois servidores homogêneos e heterogêneos.

RESULTADO 4.6: O tempo médio no sistema $S(\lambda, \mu, 1,2) \quad$ é maior que o tempo médio no sistema $S(\lambda, \mu, 1,1)$ correspondente.

\section{DEMONSTRAÇÃO:}

O tempo médio no sistema $S(\lambda, \mu, 1,1)$ é dado por

$$
T_{1}=\frac{1}{\mu-\lambda}
$$

e o tempo médio no sistema $S(\lambda, \mu, 1,2)$ é dado por

$$
T_{2}=T_{s}^{2}\left(\mu_{1}, \mu_{2}\right)
$$

Basta então provar que $T_{2}^{*}=T_{s}^{2}\left(\mu_{1}^{*}, \mu_{2}^{*}\right)>T_{1}$

$$
T_{2}^{*}>T_{1}
$$

$$
\begin{aligned}
\Longleftrightarrow & \frac{\mu^{3} \cdot \sqrt{\lambda} \sqrt{\lambda+\mu}}{(\mu-\lambda)\left[(\mu+\lambda-\sqrt{\lambda} \sqrt{\lambda+\mu})(-\lambda+\sqrt{\lambda} \sqrt{\lambda+\mu})(2 \lambda+\mu)(\mu-\lambda)+\mu^{2} \lambda \sqrt{\lambda} \sqrt{\lambda+\mu}\right]} \\
& >\frac{1}{\mu-\lambda} \\
\Longleftrightarrow & \mu^{3} \sqrt{\lambda} \sqrt{\lambda+\mu} \\
& >(\mu+\lambda-\sqrt{\lambda} \sqrt{\lambda+\mu})(-\lambda+\sqrt{\lambda} \sqrt{\lambda+\mu})(2 \lambda+\mu)(\mu-\lambda)+\mu^{2} \lambda \sqrt{\lambda} \sqrt{\lambda+\mu} \\
\Longleftrightarrow & \mu^{2} \sqrt{\lambda} \sqrt{\lambda+\mu}(\mu-\lambda)>(\mu+\lambda-\sqrt{\lambda} \sqrt{\lambda+\mu})(-\lambda+\sqrt{\lambda} \sqrt{\lambda+\mu})(2 \lambda+\mu)(\mu-\lambda)
\end{aligned}
$$




$$
\begin{aligned}
& \Longleftrightarrow \mu^{2} \sqrt{\lambda} \sqrt{\lambda+\mu}>(2 \lambda+\mu)[-2 \lambda(\lambda+\mu)+(2 \lambda+\mu) \sqrt{\lambda} \sqrt{\lambda+\mu}] \\
& \Longleftrightarrow\left(\mu^{2}-(2 \lambda+\mu)^{2}\right) \sqrt{\lambda} \sqrt{\lambda+\mu}>-2 \lambda(\lambda+\mu)(2 \lambda+\mu)
\end{aligned}
$$

Mas

$$
\begin{aligned}
& \mu^{2}-(2 \lambda+\mu)^{2}=(\mu-(2 \lambda+\mu))(\mu+(2 \lambda+\mu))=-4 \lambda(\lambda+\mu) \\
\Longleftrightarrow & -4 \lambda(\lambda+\mu) \sqrt{\lambda} \sqrt{\lambda+\mu}>-2 \lambda(\lambda+\mu)(2 \lambda+\mu) \\
\Longleftrightarrow & 2 \sqrt{\lambda} \sqrt{\lambda+\mu}<2 \lambda+\mu \quad(\text { pois } \lambda>0) \\
\Longleftrightarrow & 4 \lambda(\lambda+\mu)<4 \lambda^{2}+4 \lambda \mu+\mu^{2} \quad \\
\Longleftrightarrow & \mu^{2}>0
\end{aligned}
$$

Logo para $\mu>0$ temos $T_{2}^{*}>T_{1}$ e como $T_{2}>T_{2}^{*}$ temos que $T_{2}>T_{1}$.

RESULTADO 4.7: Um sistema heterogêneo $S(\lambda, \mu, 1,2)$ é melhor que o correspondente sistema homogêneo se e somente se

$$
\rho<\frac{\mu_{2}}{\mu_{1}-\mu_{2}}
$$

onde

$\mu_{i}$ - capacidade de atendimento do i-ésimo servidor no sistema heterogêneo, $i=1,2$.

Observação: O sistema heterogêneo é dito melhor que o sistema homogêneo se

$$
p_{o}^{H}>p_{o} \quad \text { e } \quad p_{i}^{H}<p_{i}, i \geq 1
$$

onde, $\operatorname{para} j \geq 0$

$p_{j}^{H}$ - probabilidade de existirem $j$ jobs no sistema heterogêneo em equilíbrio.

$p_{j}$ - probabilidade de existirem $j$ jobs no sistema homogêneo em equilíbrio.

Note-se que este conceito é bastante amplo, pois está relacionado com o sistema como um todo e não apenas com tempo médio no sistema ou com tempo médio na fila do sistema.

\section{DEMONSTRAÇÃO:}

Para o sistema heterogêneo valem as seguintes expressões, obtidas a partir das equações de equilíbrio do sistema:

$$
p_{2}^{H}=\rho p_{1}^{H}
$$




$$
\begin{aligned}
p_{i}^{H} & =\rho^{i-2} p_{2}^{H}=\rho^{i-1} p_{1}^{H} \quad i \geq 2 \\
p_{1}^{H} & =\frac{\lambda\left(\lambda+\mu_{2}\right)}{\mu_{1} \mu_{2}} \frac{1}{2 \rho+1} p_{o}^{H} \\
& =\left[\frac{\mu_{1} \mu_{2}(2 \rho+1)}{\lambda\left(\lambda+\mu_{2}\right)}+\frac{1}{1-\rho}\right]^{-1}
\end{aligned}
$$

Logo, para o sistema homogêneo correspondente, vale que:

$$
\begin{aligned}
p_{i} & =\rho^{i-1} p_{1} \quad i \geq 2 \\
p_{1} & =2 \rho p_{o} \\
& =\left[\frac{\mu^{2}}{\lambda\left(\lambda+\frac{\mu}{2}\right)}(2)^{-1}+\frac{1}{1-\rho}\right]^{-1}=\left[\frac{1}{2 \rho}+\frac{1}{1-\rho}\right]^{-1}
\end{aligned}
$$

Portanto

$$
\begin{aligned}
p_{1}^{H}<p_{1} & \Longleftrightarrow\left[\frac{\mu_{1} \mu_{2}(2 \rho+1)}{\lambda\left(\lambda+\mu_{2}\right)}+\frac{1}{1-\rho}\right]^{-1}<\left[\frac{1}{2 \rho}+\frac{1}{1-\rho}\right]^{-1} \\
& \Longleftrightarrow \frac{\mu_{1} \mu_{2}(2 \rho+1)}{\lambda\left(\lambda+\mu_{2}\right)}>\frac{1}{2 \rho} \\
& \Longleftrightarrow \frac{\lambda\left(\lambda+\mu_{2}\right)}{\mu_{1} \mu_{2}(2 \rho+1)}<2 \rho
\end{aligned}
$$

Seja $q=\frac{\mu_{1}}{\mu_{2}}$, temos:

$$
\begin{aligned}
& \rho=\frac{\lambda}{\mu_{1}^{\lambda} \mu_{2}}=\frac{\lambda}{\mu_{1}} \frac{1}{\left(1+\frac{1}{q}\right)} \Rightarrow \frac{\lambda}{\mu_{1}}=\frac{(q+1)}{q} \rho \\
& \rho=\frac{\lambda}{\mu_{1}+\mu_{2}}=\frac{\lambda}{\mu_{2}} \frac{1}{q+1} \Rightarrow \frac{\lambda}{\mu_{2}}=(q+1) \rho
\end{aligned}
$$

E ainda

$$
\begin{aligned}
p_{1}^{H}<p_{1} & \Longleftrightarrow \frac{\lambda}{\mu_{1}}\left(\frac{\lambda}{\mu_{2}}+1\right)<2 \rho(1+2 \rho) \\
& \Longleftrightarrow \frac{(q+1)}{q} \rho((q+1) \rho+1)<2 \rho(1+2 \rho) \\
& \Longleftrightarrow(q+1)^{2} \rho+(q+1)<2 q(1+2 \rho) \\
& \Longleftrightarrow\left((q+1)^{2}-4 q\right) \rho<q-1 \\
& \Longleftrightarrow(q-1)^{2} \rho<q-1 \\
& \left.\Longleftrightarrow \rho<\frac{1}{q-1} \quad \text { (pois } q \geq 1\right) \quad \text { s}_{o}^{\prime} \\
& \Longleftrightarrow \rho<\frac{\mu_{2}}{\mu_{1}-\mu_{2}}
\end{aligned}
$$


Vale também que para $i \geq 2$

$$
\begin{aligned}
p_{i}^{H}<p_{i} & \Longleftrightarrow \rho^{i-1} p_{1}^{H}<\rho^{i-1} p_{1} \\
& \Longleftrightarrow p_{1}^{H}<p_{1} \quad(\text { pois } \rho>0)
\end{aligned}
$$

Resta apenas provar que $p_{o}^{H}>p_{o} \Longleftrightarrow \rho<\frac{\mu_{2}}{\mu_{1}-\mu_{2}}$.

De (1) e (2) obtemos que

$$
\begin{aligned}
p_{o}^{H} & =\frac{\mu_{1} \mu_{2}(2 \rho+1)}{\lambda\left(\lambda+\mu_{2}\right)}\left[\frac{\mu_{1} \mu_{2}(2 \rho+1)}{\lambda\left(\lambda+\mu_{2}\right)}+\frac{1}{1-\rho}\right]^{-1}= \\
& =\frac{\mu_{1} \mu_{2}(2 \rho+1)}{\lambda\left(\lambda+\mu_{2}\right)}\left[\frac{\mu_{1} \mu_{2}(2 \rho+1)(1-\rho)+\lambda\left(\lambda+\mu_{2}\right)}{\lambda\left(\lambda+\mu_{2}\right)(1-\rho)}\right]^{-1}= \\
& =\frac{\mu_{1} \mu_{2}(2 \rho+1)(1-\rho)}{\mu_{1} \mu_{2}(2 \rho+1)(1-\rho)+\lambda\left(\lambda+\mu_{2}\right)}
\end{aligned}
$$

De (3) e (4) obtemos que

$$
p_{o}=\frac{1}{2 \rho}\left[\frac{1}{2 \rho}+\frac{1}{1-\rho}\right]^{-1}=\frac{1-\rho}{1+\rho}
$$

Portanto

$$
\begin{aligned}
p_{o}^{H}>p_{o} & \Longleftrightarrow \frac{\mu_{1} \mu_{2}(2 \rho+1)}{\mu_{1} \mu_{2}(2 \rho+1)(1-\rho)+\lambda\left(\lambda+\mu_{2}\right)}>\frac{1}{1+\rho} \\
& \Longleftrightarrow \mu_{1} \mu_{2}(2 \rho+1)(1+\rho)>\mu_{1} \mu_{2}(2 \rho+1)(1-\rho)+\lambda\left(\lambda+\mu_{2}\right) \\
& \Longleftrightarrow \mu_{1} \mu_{2}(2 \rho+1) 2 \rho>\lambda\left(\lambda+\mu_{2}\right) \\
& \Longleftrightarrow \rho<\frac{\mu_{2}}{\mu_{1}-\mu_{2}}
\end{aligned}
$$

FATO 4.8: O corolário 4.5 é um caso particular do resultado 4.7.

DEMONSTRAÇÃO:

As capacidades de atendimento dos servidores no sistema heterogêneo ótimo são dadas por

$$
\mu_{1}^{*}=\lambda \sqrt{1+\frac{1}{\rho}}\left(\sqrt{1+\frac{1}{\rho}}-1\right) \quad \text { e } \mu_{2}^{*}=\lambda\left(\sqrt{1+\frac{1}{\rho}}-1\right)
$$




$$
\begin{aligned}
\rho<\frac{\mu_{2}^{*}}{\mu_{1}^{*}-\mu_{2}^{*}} & \Longleftrightarrow \rho<\frac{\lambda\left(\sqrt{1+\frac{1}{\rho}}-1\right)}{\lambda \sqrt{1+\frac{1}{\rho}}\left(\sqrt{1+\frac{1}{\rho}}-2\right)+\lambda} \\
& \Longleftrightarrow \rho+\rho \sqrt{1+\frac{1}{\rho}}\left(\sqrt{1+\frac{1}{\rho}}-2\right)<\sqrt{1+\frac{1}{\rho}}-1 \\
& \Longleftrightarrow \rho+\rho\left(1+\frac{1}{\rho}\right)-2 \rho \sqrt{1+\frac{1}{\rho}}<\sqrt{1+\frac{1}{\rho}}-1 \\
& \Longleftrightarrow 2(\rho+1)<\sqrt{1+\frac{1}{\rho}}(2 \rho+1) \\
& \Longleftrightarrow 4(\rho+1)^{2}<\frac{\rho+1}{\rho}(2 \rho+1)^{2}=4 \rho^{2}+4 \rho+1+4 \rho+4+\frac{1}{\rho} \\
& \Longleftrightarrow 1+\frac{1}{\rho}>0
\end{aligned}
$$

Portanto o sistema heterogêneo com distribuição ótima de capacidades é melhor que o correspondente sistema homogêneo.

\section{Análise do comportamento de $\frac{\mu_{1}^{*}}{\mu_{2}^{*}}$ em função de $\rho$}

Um outro ponto de interesse é o estudo da variação da distribuição ótima de capacidades entre os servidores em função da utilização do sistema.

Vimos que a distribuição ótima de capacidades é definida por

$$
\begin{aligned}
& \mu_{1}^{*}=\mu+\lambda-\sqrt{\lambda} \sqrt{\lambda+\mu} \\
& \mu_{2}^{*}=-\lambda+\sqrt{\lambda} \sqrt{\lambda+\mu}
\end{aligned}
$$

ou equivalentemente

$$
\begin{aligned}
& \mu_{1}^{*}=\lambda \sqrt{1+\frac{1}{\rho}}\left(\sqrt{1+\frac{1}{\rho}}-1\right) \\
& \mu_{2}^{*}=\lambda\left(\sqrt{1+\frac{1}{\rho}}-1\right)
\end{aligned}
$$

Portanto $\frac{\mu_{1}^{*}}{\mu_{2}^{*}}=\sqrt{1+\frac{1}{\rho}}$

Esta expressão nos permite concluir facilmente que nunca teremos uma alocação ótima de capacidades com servidores iguais, o que já foi demonstrado anteriormentee.

Além disso, podemos esquematizar graficamente $\frac{\mu_{1}^{*}}{\mu_{2}^{*}}$ em função de $\rho$. 
FATO 4.9: Seja $Q(\rho)=\frac{\mu_{1}^{*}}{\mu_{2}^{*}}(\rho)$
a) $\lim _{\rho \rightarrow 1} Q(\rho)=\sqrt{2}$
b) $\lim _{\rho \rightarrow 0} Q(\rho)=+\infty$
c) $Q(\rho)$ é estritamente decrescente em ]0, 1[
d) $Q(\rho)$ é convexa em ]0,1[

\section{DEMONSTRAÇÃO:}

As demonstrações dos itens a), b) e c) são imediatas.

Demonstração do ítem d):

$$
\begin{gathered}
Q^{\prime}(\rho)=\frac{1}{2}\left(1+\frac{1}{\rho}\right)^{-1 / 2}\left(-\frac{1}{\rho^{2}}\right)=\frac{-1}{2 \rho^{2}}\left(1+\frac{1}{\rho}\right)^{-1 / 2} \\
Q^{\prime \prime}(\rho)=\frac{1}{\rho^{3}}\left(1+\frac{1}{\rho}\right)^{-1 / 2}-\frac{1}{2 \rho^{2}}\left(\frac{1}{2 \rho^{2}}\left(1+\frac{1}{\rho}\right)^{-3 / 2}\right) \\
\text { seja } A=\left(1+\frac{1}{\rho}\right)^{-1 / 2} \\
=\frac{1}{\rho^{3}} A-\frac{1}{4 \rho^{4}} A^{3}=\frac{A}{4 \rho^{4}}\left(4 \rho-A^{2}\right) \\
=\frac{A}{4 \rho^{4}}(2 \sqrt{\rho}-A)(2 \sqrt{\rho}+A) \\
2 \sqrt{\rho}-A-2 \sqrt{\rho}-\left(1+\frac{1}{\rho}\right)^{-1 / 2}=\frac{\sqrt{\rho}}{\sqrt{\rho+1}}(2 \sqrt{\rho+1}-1) \\
2 \sqrt{\rho}-A \geq 0 \Longleftrightarrow 2 \sqrt{\rho+1} \geq 1 \\
\Longleftrightarrow \rho+1 \geq 1 / 4
\end{gathered}
$$

Como $\rho \in] 0,1\left[\right.$ temos $Q^{\prime \prime}(\rho)>0$. 
O gráfico de $Q(\rho)$ tem então a seguinte forma:

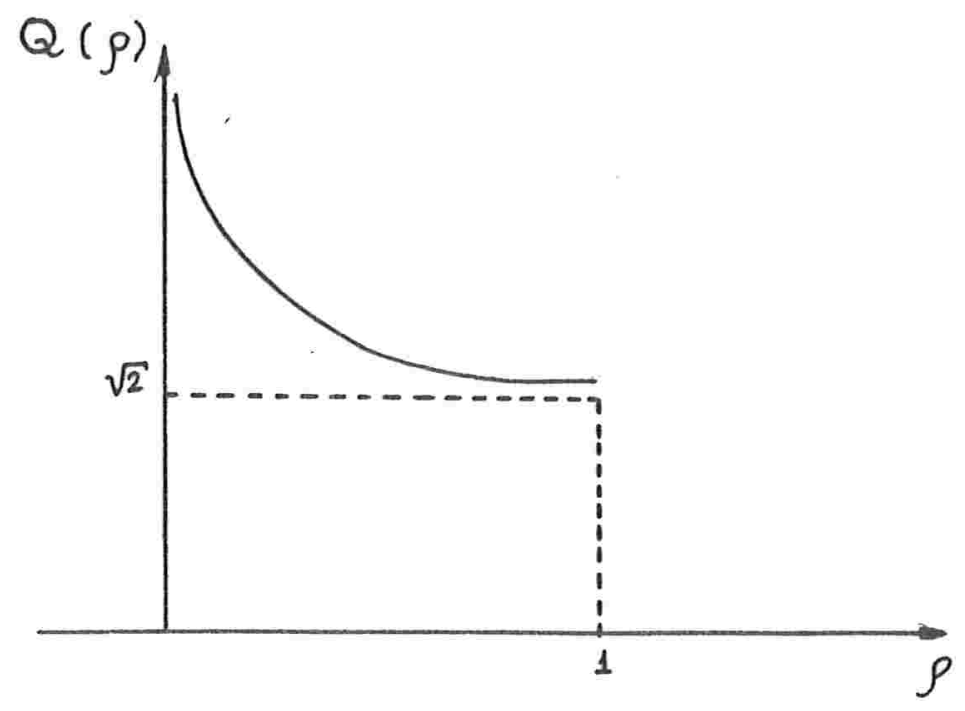

Análise do comportamento de $T_{2}^{*}$ como função de $\lambda$

O objetivo dessa análise é verificar como a função que determina o menor tempo médio no sistema que consiste de uma fila $M / M / 2($ sistema $S(\lambda, \mu, 1,2)$ ) se comporta com a variação da carga no sistema.

Vamos denotar por $T_{2}^{*}(\lambda)$ ao valor ótimo obtido para o problema $P$, definido anteriormente, considerando o sistema $S(\lambda, \mu, 1,2)$ com $\mu>0$.

Para $\lambda>0$ foram obtidos os valores $\mu_{1}^{*}$ e $\mu_{2}^{*}$ e portanto $T_{2}^{*}(\lambda)=T_{s}^{2}\left(\mu_{1}^{*}, \mu_{2}^{*}\right)$.

Resta calcular $T_{2}^{*}(0)$

Temos que $T_{2}^{*}(0)=\min _{\mu_{1}, \mu_{2}} \lim _{\lambda \rightarrow 0} T_{s}^{2}$.

Mas $\lim _{\lambda \rightarrow 0} T_{s}^{2}=\frac{1}{\mu_{1}}$, que vai atingir o valor mínimo em $\mu_{1}^{*}=\mu$ e $\mu_{2}^{*}=0$, isto é, com apenas um servidor.

Portanto $T_{2}^{*}(0)=\frac{1}{\mu}$, que é o tempo médio no sistema $M / M / 1$ com carga nula $(S(0, \mu, 1,1))$.

Este mesmo resultado pode ser verificado calculando-se $\lim _{\lambda \rightarrow 0} T_{2}^{*}$, onde $T_{2}^{*}=T_{s}^{2}\left(\mu_{1}^{*}, \mu_{2}^{*}\right)$, pois $\lim _{\lambda \rightarrow 0} T_{2}^{*}=\lim _{\lambda \rightarrow 0} \min _{\mu_{1}, \mu_{2}} T_{s}^{2}$. 
Temos então

$$
\begin{aligned}
T_{2}^{*} & =\frac{\mu^{3} \sqrt{\lambda} \sqrt{\lambda+\mu}}{(\mu-\lambda)\left[(-2(\lambda+\mu) \lambda+(2 \lambda+\mu) \sqrt{\lambda} \sqrt{\lambda+\mu})(2 \lambda+\mu)(\mu-\lambda)+\mu^{2} \lambda \sqrt{\lambda} \sqrt{\lambda+\mu}\right]} \\
& =\frac{\mu^{3}}{-2 \sqrt{\lambda} \sqrt{\lambda+\mu}(2 \lambda+\mu)(\mu-\lambda)^{2}+(2 \lambda+\mu)^{2}(\mu-\lambda)^{2}+\mu^{2} \lambda(\mu-\lambda)}
\end{aligned}
$$

Portanto

$$
\lim _{\lambda \rightarrow 0} T_{2}^{*}=\frac{\mu^{3}}{\mu^{2} \mu^{2}}=\frac{1}{\mu}
$$

Podemos também verificar facilmente que

$$
\lim _{\lambda \rightarrow \mu} T_{2}^{*}(\lambda)=+\infty
$$

As figuras 4.1 e 4.2 mostram resultados gráficos para $T_{2}^{*}(\lambda)$ para alguns valores de $\mu$. $\mathrm{Na}$ figura 4.1, são traçados os seguintes gráficos para tempo médio no sistema considerando $\mu=8.0$ :

- Gráfico para o sistema $S(\lambda, 8.0,1,2)$ com servidores iguais (sistema homogêneo $S(\lambda, 8.0,1,2))$. O tempo médio no sistema, neste caso, é dado por

$$
\frac{1}{\frac{\mu}{2}\left(1-\rho^{2}\right)}=\frac{1}{4.0\left(1-\frac{\lambda^{2}}{8.0^{2}}\right)}
$$

- Gráfico para o sistema com distribuição ótima de capacidades nos servidores. O tempo médio no sistema é dado por $T_{2}^{*}(\lambda)$.

- Gráfico para o sistema $S(\lambda, 8.0,1,1)$, que é o sistema de um servidor correspondente a.os sistemas de dois servidores $S(\lambda, 8.0,1,2)$. O tempo médio no sistema é dado por

$$
\frac{1}{\mu-\lambda}=\frac{1}{8.0-\lambda}
$$

Podemos observar nesta figura que:

- O sistema com distribuição ótima de capacidades não é homogêneo.

- Um sistema com dois servidores (homogêneo ou com distribuição ótima de capacidades) tem desempenho pior que o sistema de um servidor correspondente.

Na figura 4.2 são traçados gráficos de $T_{2}^{*}(\lambda)$ para $\mu=4.0$ e $\mu=8.0$.

Podemos ainda observar nos gráficos de $T_{2}^{*}(\lambda)$, tanto na figura 4.1 quanto na figura 4.2, que a função $T_{2}^{*}$ tem um ponto de inflexão para um valor $\lambda=\bar{\lambda}$, dependente de $\mu$, sendo a função côncava até $\bar{\lambda}$ e convexa a partir de $\bar{\lambda}$. 


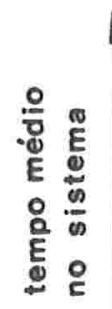

FIG.4.1

0.9

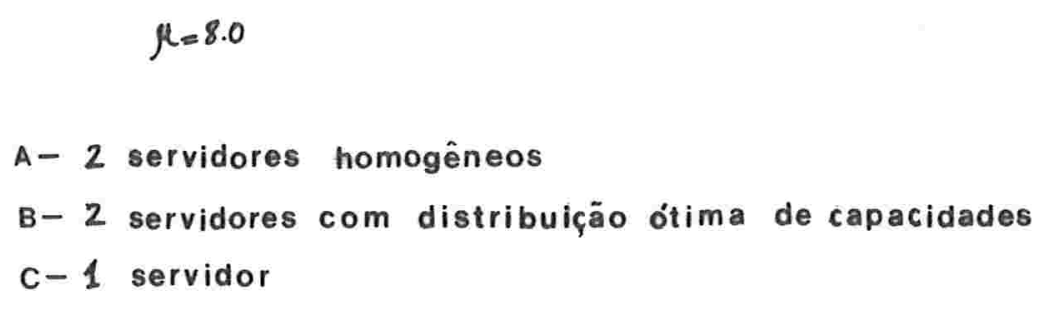

A- 2 servidores homogêneos

B- 2 servidores com distribuição ótima de capacidades

C- 1 servidor

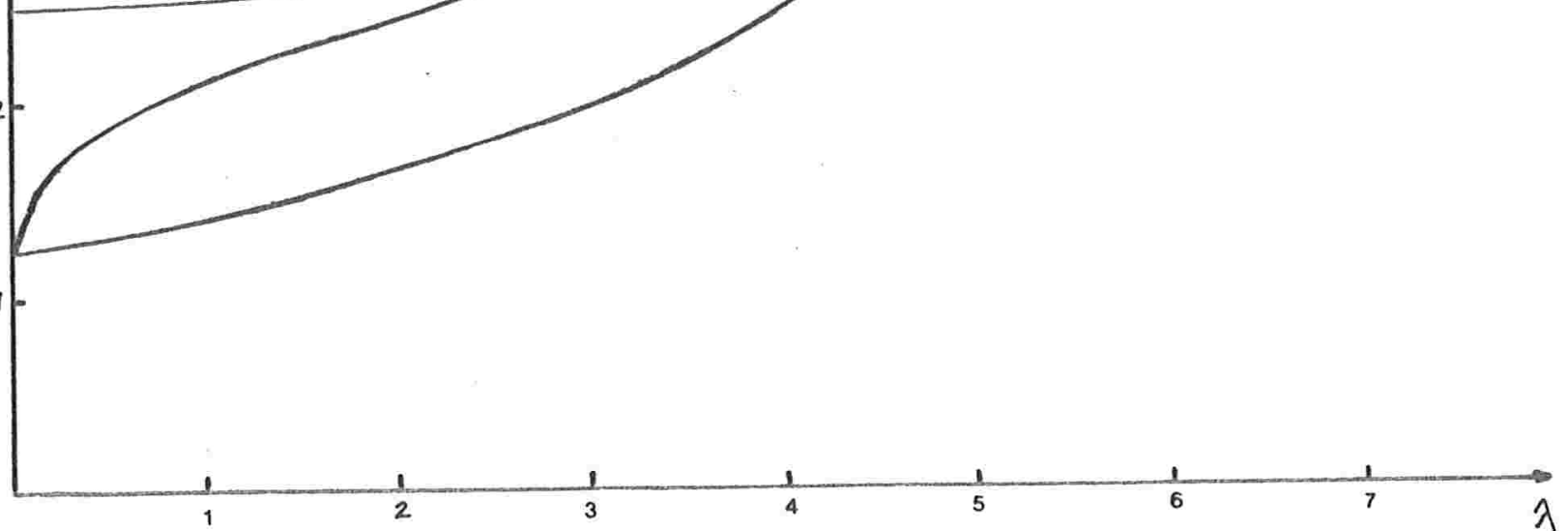




\subsubsection{Análise para tempo de fila}

Seja $N_{f}^{2}$ - número médio de jobs na fila do sistema de dois servidores

$$
\begin{aligned}
N_{f}^{2} & =1 * p_{3}+2 * p_{4}+\cdots \\
& =\sum_{i=3}^{\infty}(i-2) p_{i} \quad \text { onde } \quad p_{i}=\left(\frac{\lambda}{\mu}\right)^{i-2} p_{2}
\end{aligned}
$$

Logo

$$
\begin{aligned}
N_{f}^{2} & =p_{2} \sum_{i=3}^{\infty}(i-2)\left(\frac{\lambda}{\mu}\right)^{i-2}=p_{2} \sum_{j=1}^{\infty} j\left(\frac{\lambda}{\mu}\right)^{j}=p_{2} \sum_{j=0}^{\infty} j\left(\frac{\lambda}{\mu}\right)^{j} \\
& =p_{2} \frac{\frac{\lambda}{\mu}}{\left(1-\frac{\lambda}{\mu}\right)^{2}}=p_{2} \frac{\lambda \mu}{(\mu-\lambda)^{2}} \\
& =\frac{\lambda \mu}{(\mu-\lambda)^{2}}\left[\frac{\lambda^{2}\left(\lambda+\mu_{2}\right)(\mu-\lambda)}{\mu_{1} \mu_{2}(2 \lambda+\mu)(\mu-\lambda)+\mu^{2} \lambda\left(\lambda+\mu_{2}\right)}\right] \\
& =\frac{\lambda^{3} \mu\left(\lambda+\mu_{2}\right)}{(\mu-\lambda)\left[\mu_{1} \mu_{2}(2 \lambda+\mu)(\mu-\lambda)+\mu^{2} \lambda\left(\lambda+\mu_{2}\right)\right]}
\end{aligned}
$$

$\mathrm{E}$, por Little, obtemos o tempo médio na fila do sistema com dois servidores

$$
T_{f}^{2}=\frac{\lambda^{2} \mu\left(\lambda+\mu_{2}\right)}{(\mu-\lambda)\left[\mu_{1} \mu_{2}(2 \lambda+\mu)(\mu-\lambda)+\mu^{2} \lambda\left(\lambda+\mu_{2}\right)\right]}
$$

É fácil verificar que para $\mu_{1}=\mu_{2}$, a fórmula acima fornece a conhecida expressão para cálculo de tempo médio de fila $M / M / 2$ com servidores iguais (vide [16], [30]).

$$
\begin{aligned}
\left.T_{f}^{2}\right|_{\mu_{1}=\mu_{2}} & =\frac{\lambda^{2} \mu\left(\lambda+\frac{\mu}{2}\right)}{(\mu-\lambda)\left[\frac{\mu^{2}}{4}(2 \lambda+\mu)(\mu-\lambda)+\mu^{2} \lambda\left(\lambda+\frac{\mu}{2}\right)\right]} \\
& =\frac{\frac{\lambda^{2} \mu}{2}(2 \lambda+\mu)}{(\mu-\lambda)\left[\frac{\mu^{2}}{4}(2 \lambda+\mu)(\mu-\lambda)+\frac{\mu^{2} \lambda}{2}(2 \lambda+\mu)\right]} \\
& =\frac{2 \lambda^{2} \mu}{(\mu-\lambda)\left[\mu^{2}(\mu-\lambda)+2 \mu^{2} \lambda\right]}=\frac{2 \lambda^{2}}{(\mu-\lambda) \mu(\mu+\lambda)}=\frac{2 \lambda^{2}}{\mu\left(\mu^{2}-\lambda^{2}\right)} \\
& =\frac{2 \rho^{2}}{\mu\left(1-\rho^{2}\right)}=\frac{\rho^{2}}{\frac{\mu}{2}\left(1-\rho^{2}\right)}
\end{aligned}
$$

É interessante observar que para $\mu_{2}=0$, a fórmula de $T_{f}^{2}$ se reduz à conhecida expressão para cálculo de tempo de fila de um sistema $M / M / 1$. 


$$
\left.T_{f}^{2}\right|_{\mu_{2}=0}=\frac{\lambda^{3} \mu}{(\mu-\lambda) \mu^{2} \lambda^{2}}=\frac{\lambda}{\mu(\mu-\lambda)}=\frac{\frac{\rho}{\mu}}{(1-\rho)}
$$

Isto é explicado pelo fato do cálculo de tempo de fila não envolver os estados $E_{0}, E_{1}$, $E_{2}$, onde podem ocorrer problemas quando $\mu_{2}=0$.

Vale lembrar que a fórmula de $T_{s}^{2}$ não se aplica para $\mu_{2}=0$, pois o cálculo de tempo no sistema envolve todos os estados do diagrama.

Uma vez obtida a expressão para $T_{f}^{2}$, temos interesse em obter a distribuição da capacidade total de atendimento entre os servidores que minimiza o tempo médio na fila do sistema.

Observamos que $T_{f}^{2}=\rho^{2} T_{s}^{2}$ e que portanto o par $\left(\mu_{1}^{*}, \mu_{2}^{*}\right)$ que minimiza o tempo médio no sistema também minimiza o tempo médio na fila do sistema e o número médio de jobs na fila do sistema. Este fato pode ser facilmente entendido se lembrarmos que o tempo médio no sistema é definido como o tempo médio na fila do sistema mais o tempo médio de atendimento, sendo este constante.

A seguir mostraremos que o sistema de dois servidores tem desempenho melhor que o sistema de um servidor correspondente e também um resultado relativo à comparação de sistemas de dois servidores homogêneos e heterogêneos. Estes resultados são relativos a tempo médio na fila do sistema.

RESULTADO 4.10: O tempo médio na fila de um sistema $S(\lambda, \mu, 1,2)$ é menor que o tempo médio na fila do sistema $S(\lambda, \mu, 1,1)$ correspondente.

\section{DEMONSTRAÇÃO:}

O tempo médio na fila do sistema $S(\lambda, \mu, 1,1)$ é dado por

$$
T_{1}=\frac{\lambda}{\mu(\mu-\lambda)}
$$

e o tempo médio na fila do sistema $S(\lambda, \mu, 1,2)$ é dado por

$$
T_{2}=T_{f}^{2}\left(\mu_{1}, \mu_{2}\right)
$$




$$
\begin{aligned}
T_{1}>T_{2} & \Longleftrightarrow \frac{\lambda}{\mu(\mu-\lambda)}>\frac{\lambda^{2} \mu\left(\lambda+\mu_{2}\right)}{(\mu-\lambda)\left[\mu_{1} \mu_{2}(2 \lambda+\mu)(\mu-\lambda)+\lambda^{2} \mu\left(\lambda+\mu_{2}\right)\right]} \\
& \Longleftrightarrow \mu_{1} \mu_{2}(2 \lambda+\mu)(\mu-\lambda)+\lambda^{2} \mu\left(\lambda+\mu_{2}\right)>\lambda \mu^{2}\left(\lambda+\mu_{2}\right) \\
& \Longleftrightarrow \mu_{1} \mu_{2}(2 \lambda+\mu)(\mu-\lambda)>0
\end{aligned}
$$

Como $\mu>\lambda>0$ e $\mu_{1}, \mu_{2} \geq 0$ temos que $T_{1}>T_{2}$.

RESULTADO 4.11: O sistema $S(\lambda, \mu, 1,2)$ homogêneo nunca é ótimo.

\section{DEMONSTRAÇÃO:}

Conforme já observamos $T_{f}^{2}=\rho^{2} T_{s}^{2}$ e portanto o par $\left(\mu_{1}^{*}, \mu_{2}^{*}\right)$ que minimiza o tempo médio no sistema, também minimiza o tempo médio na fila do sistema. Então pelo corolário 3.5 segue a tese.

A análise do comportamento de $\frac{\mu_{1}^{*}}{\mu_{2}^{*}}$ em função de $\rho$ feita no item análise para tempo no sistema é válida para tempo na fila do sistema, pois conforme observado, $\left(\mu_{1}^{*}, \mu_{2}^{*}\right)$ minimiza tanto o tempo médio no sistema quanto o tempo médio na fila do sistema.

\section{Análise do comportamento de $T_{f}^{*}$ como função de $\lambda$}

O objetivo desta análise é verificar como a função que determina o menor tempo médio na fila do sistema $S(\lambda, \mu, 1,2)$ se comporta com a variação da carga no sistema.

Vamos denotar por $T_{f}^{*}(\lambda)$ ao valor ótimo do problema $P_{f}$ abaixo, considerando o sistema $S(\lambda, \mu, 1,2) \operatorname{com} \mu>0$.

$$
\begin{array}{ll}
P_{f}: \quad \min & T_{f}^{2}\left(\mu_{1}, \mu_{2}\right) \\
\text { sujeito a } & \mu_{1}+\mu_{2}=\mu \\
& \mu_{1} \geq \mu_{2} \\
& \mu_{1}, \mu_{2} \geq 0
\end{array}
$$

As figuras 4.3 e 4.4 mostram resultados gráficos para $T_{f}^{*}(\lambda)$ para alguns valores de $\mu$.

$\mathrm{Na}$ figura 4.3 foram traçados os seguintes gráficos para tempo médio na fila do sistema considerando $\mu=8.0$ : 
- Gráfico para o sistema $S(\lambda, 8.0,1,2)$ com distribuição ótima de capacidades nos servidores. O tempo médio no sistema é dado por $T_{f}^{*}(\lambda)$.

- Gráfico para o sistema $S(\lambda, 8.0,1,1)$. O tempo médio na fila do sistema, para este caso, é dado por

$$
\frac{\lambda}{\mu(\mu-\lambda)}=\frac{\lambda}{8.0(8.0-\lambda)}
$$

- O gráfico para o sistema $S(\lambda, 8.0,1,2)$ homogêneo foi omitido pois os valores para tempo médio na fila deste sistema, que é dado por $\frac{\rho^{2}}{\frac{\mu}{2}\left(1-\rho^{2}\right)}$ são muito próximos aos do sistema heterogêneo ótimo, e portanto difíceis de serem representados na escala escolhida.

Podemos observar nesta figura que o sistema com dois servidores tem desempenho melhor que o sistema com um servidor correspondente.

Na figura 4.4 são traçados os gráficos para $T_{f}^{*}(\lambda)$ para $\mu=4.0$ e $\mu=10.0$.

A partir dos gráficos de $T_{f}^{*}$ nas figuras 4.3 e 4.4 podemos verificar que a função $T_{f}^{*}$ é uma função convexa em $\lambda$. 
FIG. 4.3

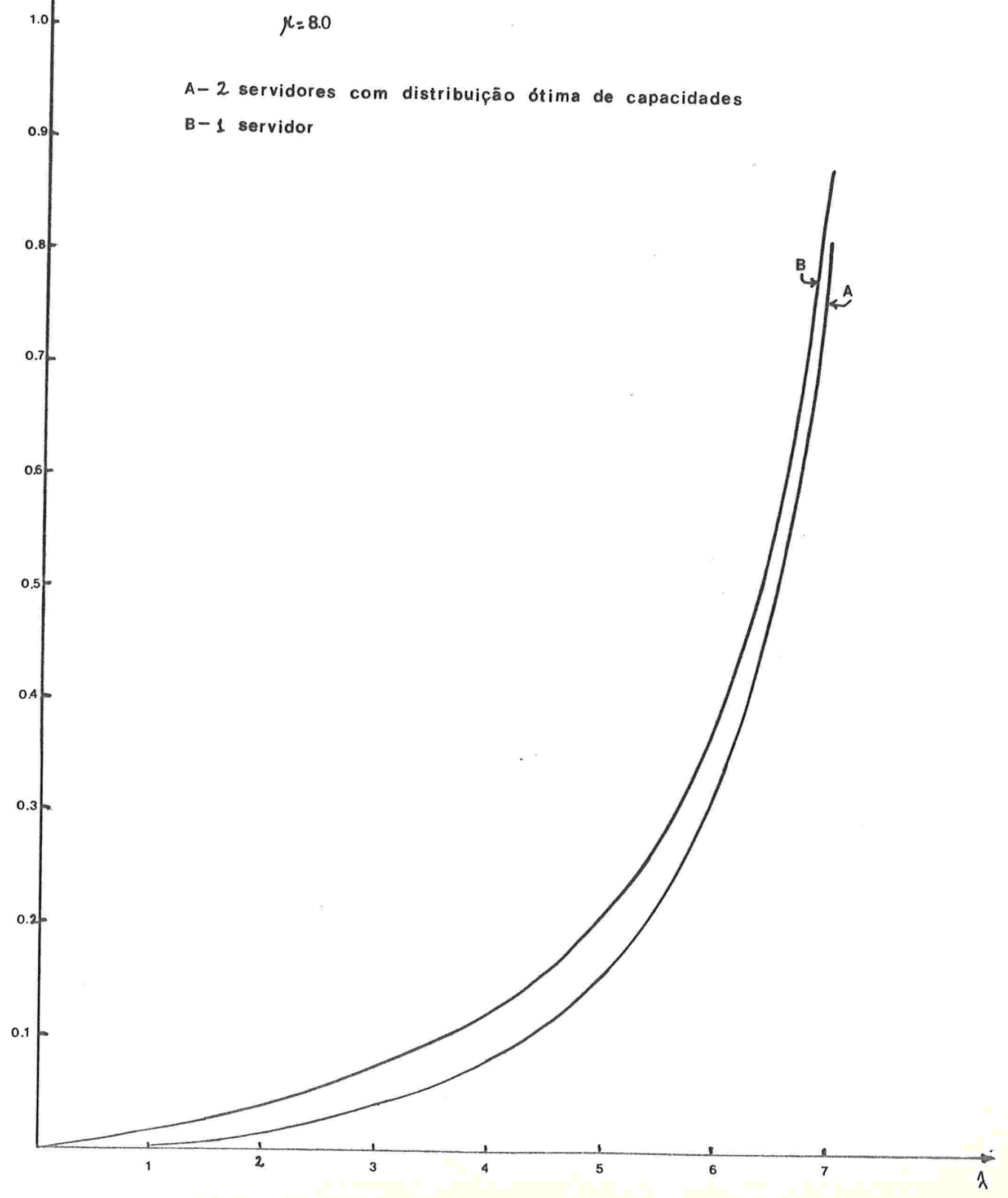


105B

FIG. 4.4

$$
\begin{aligned}
& \text { A }-\mu=4.0 \\
& \text { B- } \mu=10.0
\end{aligned}
$$

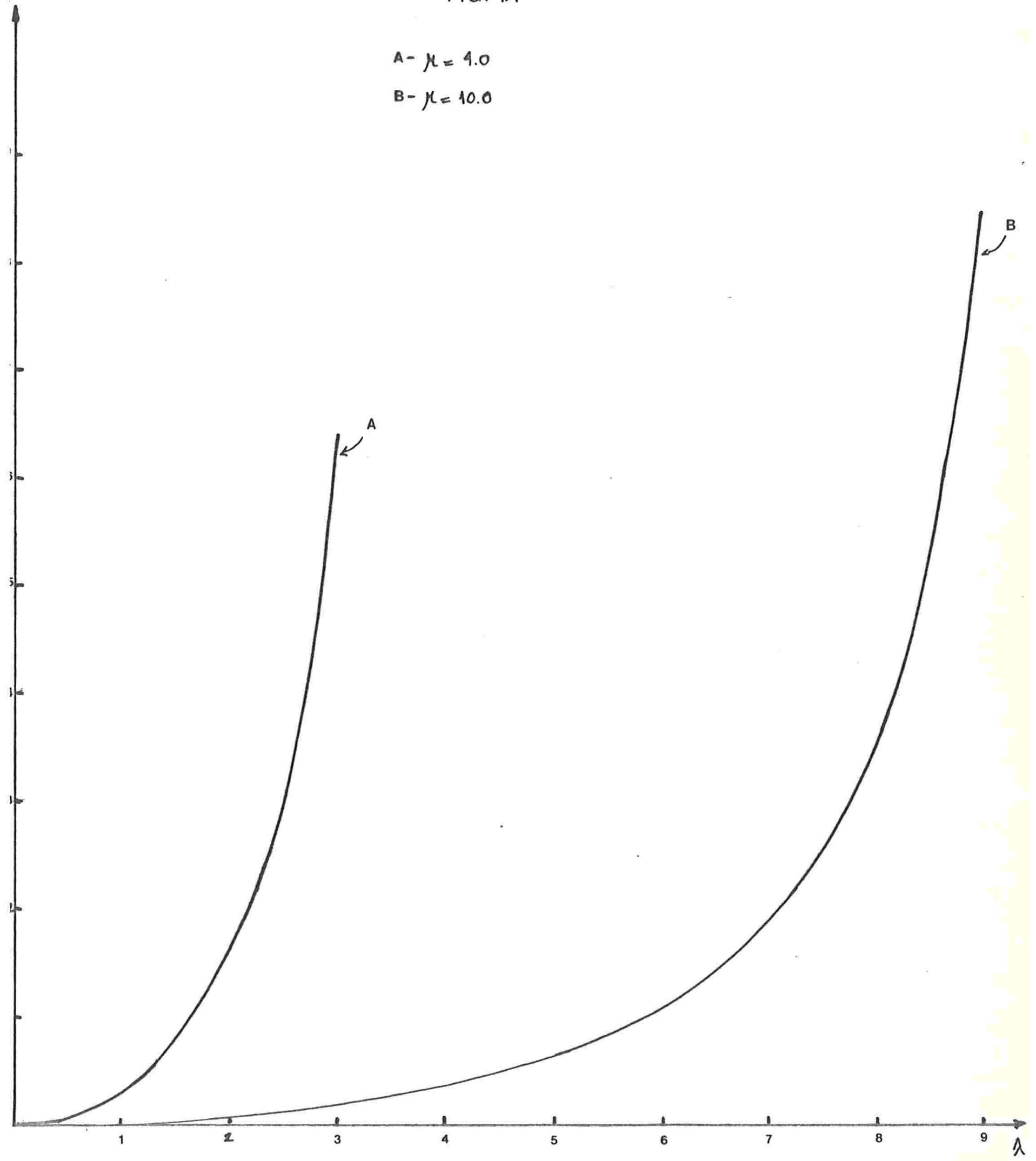




\subsection{3 - Conclusão para dois servidores}

No estudo do comportamento de um sistema $S(\lambda, \mu, 1,2)$, com servidores com capacidades não necessariamente homogêneas, foram obtidos alguns resultados importantes:

- Com relação às capacidades ótimas dos servidores, tanto para tempo médio no sistema como para tempo médio na fila do sistema:

- Nunca teremos uma distribuição ótima de capacidades com servidores homogêneos. Este fato é bastante interessante, uma vez que nos estudos de filas são sempre considerados sistemas homogêneos e portanto nunca ótimos.

- A capacidade ótima assinalada ao servidor mais 'forte' (mais rápido) decresce com o fator de utilização global do sistema.

- Com relação ao desempenho ótimo do sistema (desempenho obtido com distribuição ótima de capacidades nos servidores):

- Considerando-se a medida tempo médio no sistema, o desempenho ótimo do sistema com dois servidores é pior que o desempenho do correspondente sistema com um servidor.

- Considerando-se a medida tempo médio na fila do sistema, o desempenho ótimo do sistema com dois servidores é melhor que o desempenho do correspondente sistema com um servidor.

\section{3 - Grupo de três servidores}

O diagrama de transição de estados para o sistema $S(\lambda, \mu, 1,3)$ tem a seguinte representação: 


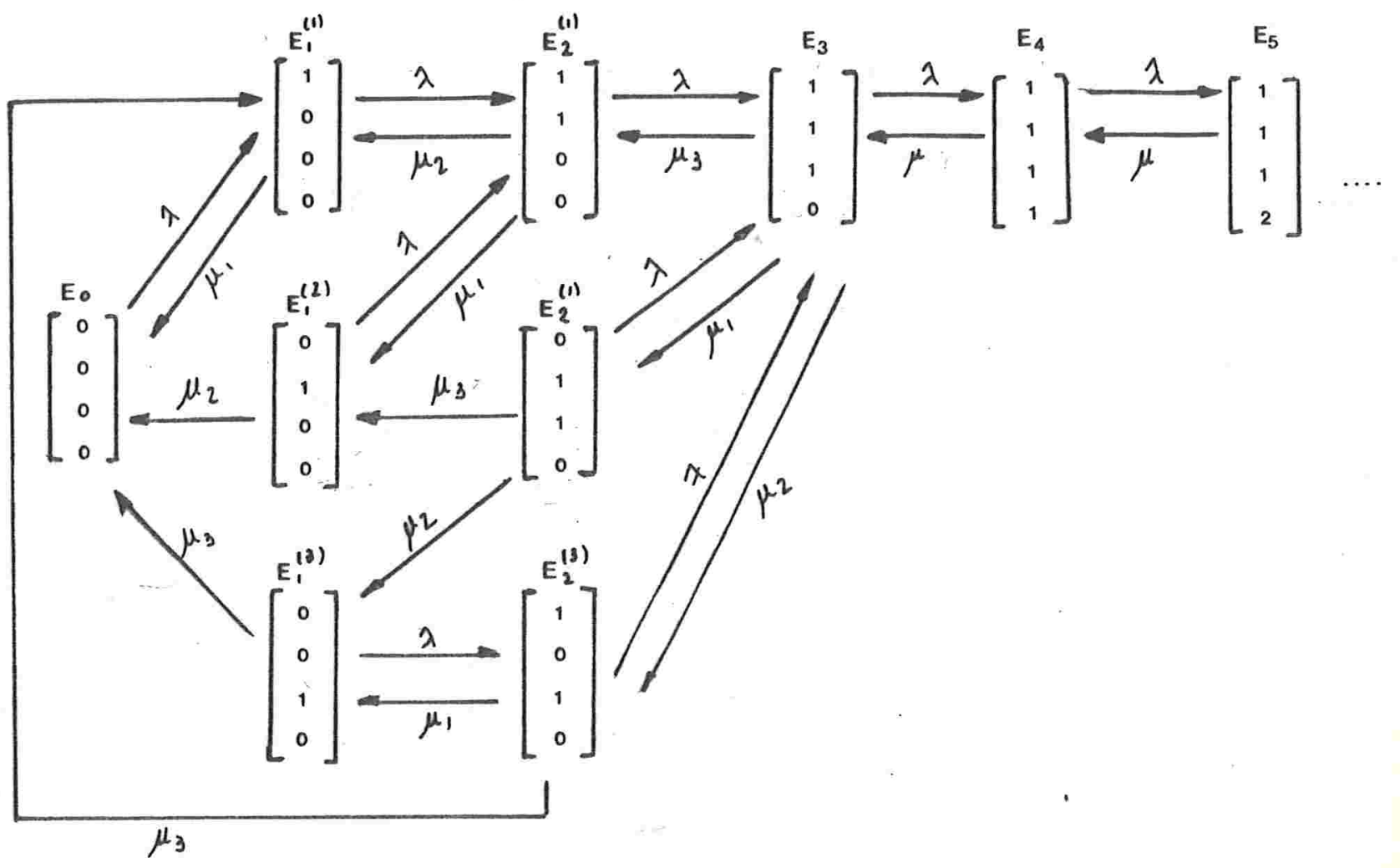

\section{OBSERVAÇÕES:}

1. A notação $E_{1}^{(j)}$ é utilizada pois existe mais de uma possibilidade de termos um elemento , no sistema.

Temos, na verdade,

$p_{1}=$ probabilidade $\left(E_{1}^{(1)}\right)+$ probabilidade $\left(E_{1}^{(2)}\right)+$ probabilidade $\left(E_{1}^{3}\right)$

$$
=p_{11}+p_{12}+p_{13}
$$

Analogamente, para dois elementos no sistema, obtemos

$$
p_{2}=p_{21}+p_{22}+p_{23}
$$

2. Para esta modelagem considera-se $\mu_{2}>0$ e $\mu_{3}>0$, uma vez que $\mu_{2}=0$ ou $\mu_{3}=0$ implicaria em 'deadlock' do sistema.

Temos então as seguintes equações de equilíbrio:

$\operatorname{Sejam} \ell_{i}=\lambda+\mu_{i} \quad i=1,2,3 \quad \mathrm{e}$

$$
\ell_{i j}=\lambda+\mu_{i}+\mu_{j} \quad i=1,2,3 \quad j=1,2,3
$$


Equação para o estado $E_{0}$ :
$\mathrm{A}:-\lambda p_{0}+\mu_{1} p_{11}+\mu_{2} p_{12}+\mu_{3} p_{13}$
$=0$

Equações para o estado $E_{1}$ :
B: $\lambda p_{0}-l_{1} p_{11}$
$+\mu_{2} p_{21}$
$+\mu_{3} p_{23}$
$=0$
$\mathrm{C}$ :
$-l_{2} p_{12}$
$+\mu_{1} p_{21}+\mu_{3} p_{22}$
$=0$
D: $-l_{3} p_{13}$
$+\mu_{2} p_{22}+\mu_{1} p_{23}$
$=0$

Equações para o estado $E_{2}$ :

$\mathrm{E}:$

$$
\lambda p_{11}+\lambda p_{12}
$$

$-l_{12} p_{21}$

$+\mu_{3} p_{3}=0$

F:

$-l_{23} p_{22}$

$+\mu_{1} p_{3}=0$

G:

$\lambda p_{13}$

$-l_{13} p_{23} \quad+\mu_{2} p_{3}=0$

Equação para o estado $E_{i}, i \geq 3$ :

$\mathrm{H}$ :

$$
\begin{array}{lll}
\lambda p_{i-1} & -(\lambda+\mu) p_{i} \quad+\mu p_{i+1}
\end{array}
$$$$
=0
$$

Passamos à resolução do sistema de equações, determinando os coeficientes $c_{i j}$, tais que $p_{i j}=c_{i j} * p_{3}$ e os coeficientes $c_{i}$, tais que $p_{i}=c_{i} * p_{3}$.

Da equação $\mathrm{F}$ vem

$$
p_{22}=\frac{\mu_{1}}{\lambda+\mu_{2}+\mu_{3}} p_{3} \quad \text { e } \quad c_{22}=\frac{\mu_{1}}{\lambda+\mu_{2}+\mu_{3}}
$$

Somando as equações de $\mathrm{A}$ a $\mathrm{G}$, temos

$-\lambda p_{21}-\lambda p_{22}-\lambda p_{23}+\mu p_{3}=0$

$$
\Rightarrow p_{2}=\frac{\mu}{\lambda} p_{3} \quad \text { e } \quad c_{2}=\frac{\mu}{\lambda}
$$

Obtemos $p_{13}$ e $p_{23}$ a partir do sistema formado pelas equações $\mathrm{D}$ e $\mathrm{G}$

$$
\left\{\begin{array}{r}
-\left(\lambda+\mu_{3}\right) p_{13}+\mu_{1} p_{23}+\mu_{2} p_{22}=0 \\
\lambda p_{13}-\left(\lambda+\mu_{1}+\mu_{3}\right) p_{23}+\mu_{2} p_{3}=0
\end{array}\right.
$$

Da segunda equação vem

$$
p_{13}=\frac{1}{\lambda}\left(\left(\lambda+\mu_{1}+\mu_{3}\right) p_{23}-\mu_{2} p_{3}\right)
$$

E substituindo na primeira 


$$
\begin{aligned}
-\left(\lambda+\mu_{3}\right) \frac{1}{\lambda}\left(\left(\lambda+\mu_{1}+\mu_{3}\right) p_{23}-\mu_{2} p_{3}\right)+\mu_{1} p_{23}+\mu_{2} p_{22}=0 \\
\Rightarrow\left[\frac{\left(\lambda+\mu_{3}\right)}{\lambda}\left(\lambda+\mu_{1}+\mu_{3}^{\prime}\right)-\mu_{1}\right] p_{23}=\frac{\left(\lambda+\mu_{3}\right)}{\lambda} \mu_{2} p_{3}+\mu_{2} p_{22} \\
=\left[\frac{\left(\lambda+\mu_{3}\right)}{\lambda} \mu_{2}+\mu_{2} \frac{\mu_{1}}{\lambda+\mu_{2}+\mu_{3}}\right] p_{3}
\end{aligned}
$$

Portanto

$$
c_{23}=\frac{\frac{\left(\lambda+\mu_{3}\right) \mu_{2}}{\lambda}+\frac{\mu_{2} \mu_{1}}{\lambda+\mu_{2}+\mu_{3}}}{\frac{\left(\lambda+\mu_{3}\right)}{\lambda}\left(\lambda+\mu_{1}+\mu_{3}\right)-\mu_{1}}=\frac{\left(\lambda+\mu_{3}\right) \mu_{2}+\frac{\lambda \mu_{2} \mu_{1}}{\lambda+\mu_{2}+\mu_{3}}}{\left(\lambda+\mu_{3}\right)\left(\lambda+\mu_{1}+\mu_{3}\right)-\lambda \mu_{1}}
$$

Logo,

$$
p_{13}=\frac{\left(\lambda+\mu_{1}+\mu_{3}\right)}{\lambda} p_{23}-\frac{\mu_{2}}{\lambda} p_{3}=\left[\frac{\left(\lambda+\mu_{1}+\mu_{3}\right) c_{23}-\mu_{2}}{\lambda}\right] p_{3}
$$

Portanto

$$
c_{13}=\frac{\left(\lambda+\mu_{1}+\mu_{3}\right) c_{23}-\mu_{2}}{\lambda}
$$

Mas $\quad p_{2}=p_{21}+p_{22}+p_{23}=c_{2} p_{3}$

$$
\Rightarrow p_{21}=\left(c_{2}-c_{22}-c_{23}\right) p_{3}
$$

Portanto $c_{21}=c_{2}-c_{22}-c_{23}$

Somando as equações A, B, C e D temos

$-\lambda p_{1}+\left(\mu_{1}+\mu_{2}\right) p_{21}+\left(\mu_{2}+\mu_{3}\right) p_{22}+\left(\mu_{1}+\mu_{3}\right) p_{23}=0$

$-\lambda p_{1}+\left[\left(\mu_{1}+\mu_{2}\right) c_{21}+\left(\mu_{2}+\mu_{3}\right) c_{22}+\left(\mu_{1}+\mu_{3}\right) c_{23}\right] p_{3}=0$

$$
\Rightarrow c_{1}=\frac{\left(\mu_{1}+\mu_{2}\right) c_{21}+\left(\mu_{2}+\mu_{3}\right) c_{22}+\left(\mu_{1}+\mu_{3}\right) c_{23}}{\lambda}
$$

Da equação $\mathrm{C}$ vem

$$
\begin{aligned}
& -\left(\lambda+\mu_{2}\right) p_{12}+\mu_{1} p_{21}+\mu_{3} p_{22}=0 \\
& \left(\lambda+\mu_{2}\right) p_{12}=\left(\mu_{1} c_{21}+\mu_{3} c_{22}\right) p_{3}
\end{aligned}
$$

$$
\Rightarrow c_{12}=\frac{\mu_{1} c_{21}+\mu_{3} c_{22}}{\lambda+\mu_{2}}
$$


Da equação $\mathrm{E}$ vem

$$
\begin{aligned}
& \lambda p_{11}+\lambda p_{12}-\left(\lambda+\mu_{1}+\mu_{2}\right) p_{21}+\mu_{3} p_{3}=0 \\
& \lambda p_{11}=\left[\left(\lambda+\mu_{1}+\mu_{2}\right) c_{21}-\lambda c_{12}-\mu_{3}\right] p_{3} \\
& \quad \Rightarrow c_{11}=\frac{1}{\lambda}\left(\left(\lambda+\mu_{1}+\mu_{2}\right) c_{21}-\lambda c_{12}-\mu_{3}\right)
\end{aligned}
$$

Da equação A vem

$$
-\lambda p_{0}+\mu_{1} p_{11}+\mu_{2} p_{12}+\mu_{3} p_{13}=0
$$

$$
\Rightarrow c_{0}=\frac{1}{\lambda}\left(\mu_{1} c_{11}+\mu_{2} c_{12}+\mu_{3} c_{13}\right)
$$

Para os estados $j \geq 4$ temos

A equação $\mathrm{H}$ para $i=3$ fica

$$
\begin{aligned}
& \lambda p_{2}-(\lambda+\mu) p_{3}+\mu p_{4}=0 \\
& \mu p_{3}-(\lambda+\mu) p_{3}+\mu p_{4}=0
\end{aligned}
$$

$$
\Rightarrow c_{4}=\frac{\lambda}{\mu}
$$

A equação $H$ para $i=4$ fica

$$
\begin{aligned}
\lambda p_{3}-(\lambda+\mu) p_{4}+\mu p_{5}=0 & \\
{\left[\lambda-(\lambda+\mu) \frac{\lambda}{\mu}\right] p_{3}+\mu p_{5}=-\frac{\lambda^{2}}{\mu} p_{3}+\mu p_{5} } & =0 \\
\Rightarrow c_{5} & =\left(\frac{\lambda}{\mu}\right)^{2}
\end{aligned}
$$

E de forma geral

$$
c_{j} \risingdotseq\left(\frac{\lambda}{\mu}\right)^{j-3}
$$

Temos que

$$
\begin{gathered}
1=\sum_{i=1}^{\infty} p_{i}=\left(\sum_{i=1}^{\infty} c_{i}\right) * p_{3}=\left(c_{0}+c_{1}+\frac{\mu}{\lambda}+1+\sum_{i=1}^{\infty}\left(\frac{\lambda}{\mu}\right)^{i}\right) * p_{3} \\
\Rightarrow p_{3}=\left(c_{0}+c_{1}+\frac{\mu}{\mu-\lambda}+\frac{\mu}{\lambda}\right)^{-1}
\end{gathered}
$$




\subsubsection{Análise para tempo no sistema}

Seja $N_{s}^{3}$ - número médio de jobs no sistema com três servidores.

$$
\begin{aligned}
N_{s}^{3} & =\sum_{i=0}^{\infty} i p_{i}=\left(\sum_{i=0}^{\infty} i c_{i}\right) * p_{3} \\
& =\left[c_{1}+2 c_{2}+3+\sum_{i=4}^{\infty} i\left(\frac{\lambda}{\mu}\right)^{i-3}\right] p_{3} \\
& =\left[c_{1}+2 \frac{\mu}{\lambda}+3+\sum_{j=1}^{\infty}(j+3)\left(\frac{\lambda}{\mu}\right)^{j}\right] p_{3}
\end{aligned}
$$

Mas

$$
\sum_{j=1}^{\infty} j\left(\frac{\lambda}{\mu}\right)^{j}=\frac{\frac{\lambda}{\mu}}{\left(1-\frac{\lambda}{\mu}\right)^{2}} \quad \text { e } \quad \sum_{j=1}^{\infty}\left(\frac{\lambda}{\mu}\right)^{j}=\frac{\frac{\lambda}{\mu}}{1-\frac{\lambda}{\mu}}
$$

Seja $A=\frac{\lambda}{\mu}$

$$
\begin{aligned}
& \frac{2}{A}+3+3 \frac{A}{1-A}+\frac{A}{(1-A)^{2}}=\frac{2(1-A)^{2}+3 A(1-A)^{2}+3 A^{2}(1-A)+A^{2}}{A(1-A)^{2}}= \\
= & \frac{2(1-A)^{2}+A^{2}+3 A(1-A)(1-A+A)}{A(1-A)^{2}}=\frac{2(1-A)^{2}-2 A^{2}+3 A}{A(1-A)^{2}}=\frac{2-A}{A(1-A)^{2}}= \\
= & \frac{2-\frac{\lambda}{\mu}}{\frac{\lambda}{\mu}\left(1-\frac{\lambda}{\mu}\right)^{2}}=\frac{(2 \mu-\lambda) \mu^{2}}{\lambda(\mu-\lambda)^{2}}
\end{aligned}
$$

Portanto,

$$
N_{s}^{3}=\left[c_{1}+\frac{\mu^{2}(2 \mu-\lambda)}{\lambda(\mu-\lambda)^{2}}\right] * p_{3}
$$

E por Little obtemos o tempo médio no sistema com três servidores

$$
T_{s}^{3}=\frac{1}{\lambda}\left[c_{1}+\frac{\mu^{2}(2 \mu-\lambda)}{\lambda(\mu-\lambda)^{2}}\right] * p_{3}
$$

É fácil verificar que para $\mu_{1}=\mu_{2}=\mu_{3}$ a fórmula acima fornece a conhecida expressão para cálculo de tempo médio no sistema com servidores iguais (vide [16], [30]). 
A expressão para tempo médio no sistema $M / M / 3$ com servidores iguais é dada por (vide capítulo 2)

$$
\frac{3}{\mu}\left[1+\frac{3 \rho^{3}}{(1-\rho)\left(3 \rho^{2}+4 \rho+2\right)}\right]=\frac{3}{\mu} \frac{\left(2+2 \rho-\rho^{2}\right)}{(1-\rho)\left(3 \rho^{2}+4 \rho+2\right)} \quad \operatorname{com} \quad \rho=\frac{\lambda}{\mu}
$$

Para $\mu_{1}=\mu_{2}=\mu_{3}=\frac{\mu}{3}$, vale que

$$
\begin{aligned}
c_{0} & =\frac{1}{\lambda} \frac{\mu}{3} c_{1} \\
c_{1} & =2 \frac{\mu}{3} \frac{1}{\lambda} c_{2}=\frac{2}{3}\left(\frac{\mu}{\lambda}\right)^{2} \Rightarrow c_{0}=\frac{2}{9}\left(\frac{\mu}{\lambda}\right)^{3} \\
p_{3} & =\left(\frac{2}{9 \rho^{3}}+\frac{2}{3 \rho^{2}}+\frac{1}{1-\rho}+\frac{1}{\rho}\right)^{-1} \\
& =\left(\frac{2(1-\rho)+6 \rho(1-\rho)+9 \rho^{3}+9 \rho^{2}(1-\rho)}{9 \rho^{3}(1-\rho)}\right)^{-1}=\frac{9 \rho^{3}(1-\rho)}{3 \rho^{2}+4 \rho+2} \\
T_{s}^{3} & =\frac{1}{\lambda}\left(c_{1}+\frac{2-\rho}{\rho(1-\rho)^{2}}\right) p_{3}=\frac{1}{\lambda}\left(\frac{2}{3 \rho^{2}}+\frac{2-\rho}{\rho(1-\rho)^{2}}\right) p_{3} \\
& =\frac{1}{\lambda}\left(\frac{2(1-\rho)^{2}+(2-\rho) 3 \rho}{3 \rho^{2}(1-\rho)^{2}}\right) \frac{9 \rho^{3}(1-\rho)}{3 \rho^{2}+4 \rho+2}=\frac{3}{\mu} \frac{\left(2+2 \rho-\rho^{2}\right)}{(1-\rho)\left(3 \rho^{2}+4 \rho+2\right)}
\end{aligned}
$$

\section{Análise do comportamento de $T_{3}^{*}(\lambda)$ como função de $\lambda$}

O objetivo dessa análise é verificar como a função que determina o menor tempo médio no sistema $S(\lambda, \mu, 1,3)$ se comporta com a variação da carga no sistema.

Vamos denotar por $T_{3}^{*}(\lambda)$ ao valor ótimo obtido para tempo médio no sistema $S(\lambda, \mu, 1,3)$.

Temos que $T_{3}^{*}(0)=\min _{\mu_{1}, \mu_{2}, \mu_{3}} \lim _{\lambda \rightarrow 0} T_{s}^{3}$

Passamos então ao cálculo de $\lim _{\lambda \rightarrow 0} T_{s}^{3}$ :

$$
\begin{aligned}
T_{s}^{3} & =\frac{1}{\lambda}\left(c_{1}+\frac{2-\rho}{\rho(1-\rho)^{2}}\right) p_{3} \\
& =\frac{\rho}{\lambda}\left(\rho^{2} c_{1}+\frac{\rho^{2}(2-\rho)}{\rho(1-\rho)^{2}}\right) \frac{p_{3}}{\rho^{3}} \\
& =\frac{1}{\mu}\left(\rho^{2} c_{1}+\frac{\rho(2-\rho)}{(1-\rho)^{2}}\right) \frac{p_{3}}{\rho^{3}}
\end{aligned}
$$


Sendo então $P(\lambda)=\rho^{2} c_{1}+\frac{\rho(2-\rho)}{(1-\rho)^{2}} \quad$ e $\quad Q(\lambda)=\frac{p_{3}}{\rho^{3}}$ temos

$$
\lim _{\lambda \rightarrow 0} T_{s}^{3}=\frac{1}{\mu} \lim _{\lambda \rightarrow 0} P(\lambda) Q(\lambda)
$$

Mas, sabemos por [22] que se $\exists \lim _{\lambda \rightarrow 0} P(\lambda)$ e $\exists \lim _{\lambda \rightarrow 0} Q(\lambda)$ então

$$
\lim _{\lambda \rightarrow 0} T_{s}^{3}=\frac{1}{\mu} \lim _{\lambda \rightarrow 0} P(\lambda) \lim _{\lambda \rightarrow 0} Q(\lambda)
$$

Assim, passamos ao cálculo desses limites.

a) Cálculo de $\lim _{\lambda \rightarrow 0} P(\lambda)$.

Sendo $P_{1}(\lambda)=\rho^{2} c_{1}$ e $P_{2}(\lambda)=\frac{\rho(2-\rho)}{(1-\rho)^{2}}$, temos que $P(\lambda)=P_{1}(\lambda)+P_{2}(\lambda)$.

Por [22], se $\exists \lim _{\lambda \rightarrow 0} P_{1}(\lambda)$ e $\exists \lim _{\lambda \rightarrow 0} P_{2}(\lambda)$, então

$\lim _{\lambda \rightarrow 0} P(\lambda)=\lim _{\lambda \rightarrow 0} P_{1}(\lambda)+\lim _{\lambda \rightarrow 0} P_{2}(\lambda)$

a.1) Cálculo de $\lim _{\lambda \rightarrow 0} P_{1}(\lambda)$.

$$
\begin{aligned}
c_{1} & =\frac{\left(\mu_{1}+\mu_{2}\right) c_{21}+\left(\mu_{2}+\mu_{3}\right) c_{22}+\left(\mu_{1}+\mu_{3}\right) c_{23}}{\lambda} \\
& =\frac{1}{\lambda}\left[\left(\mu_{1}+\mu_{2}\right)\left(c_{2}-c_{22}-c_{23}\right)+\left(\mu_{2}+\mu_{3}\right) c_{22}+\left(\mu_{1}+\mu_{3}\right) c_{23}\right] \\
& =\frac{1}{\lambda}\left[\left(\mu_{1}+\mu_{2}\right) c_{2}+\left(\mu_{3}-\mu_{1}\right) c_{22}+\left(\mu_{3}-\mu_{2}\right) c_{23}\right] \\
P_{1}(\lambda) & =\rho^{2} c_{1}=\frac{\mu_{1}+\mu_{2}}{\mu}+\lambda^{2} \frac{\left(\mu_{3}-\mu_{1}\right)}{\mu^{2}} c_{22}+\lambda^{2} \frac{\left(\mu_{3}-\mu_{2}\right)}{\mu^{2}} c_{23}
\end{aligned}
$$

como $\lim _{\lambda \rightarrow 0} c_{22}=\frac{\mu_{1}}{\mu_{2}+\mu_{3}}$ e $\quad \lim _{\lambda \rightarrow 0} c_{23}=\frac{\mu_{2} \mu_{3}}{\mu_{3}\left(\mu_{1}+\mu_{3}\right)}$

temos $\lim _{\lambda \rightarrow 0} P_{1}(\lambda)=\frac{\mu_{1}+\mu_{2}}{\mu}$

a.2) Cálculo de $\lim _{\lambda \rightarrow 0} P_{2}(\lambda)$

$\lim _{\lambda \rightarrow 0} P_{2}(\lambda)=0$

Portanto

$$
\lim _{\lambda \rightarrow 0} P(\lambda)=\frac{\mu_{1}+\mu_{2}}{\mu}
$$

b) Cálculo de $\lim _{\lambda \rightarrow 0} Q(\lambda)$.

$$
\begin{aligned}
Q(\lambda) & =\frac{p_{3}}{\rho^{3}} \\
p_{3} & =\frac{1}{\frac{1}{1-\rho}+\frac{1}{\rho}+c_{0}+c_{1}} \Rightarrow \frac{p_{3}}{\rho^{3}}=\frac{1}{\frac{\rho^{3}}{1-\rho}+\rho^{2}+\rho^{3} c_{0}+\rho\left(\rho^{2} c_{1}\right)}
\end{aligned}
$$


Sendo $Q_{1}(\lambda)=\frac{\rho^{3}}{1-\rho}, Q_{2}(\lambda)=\rho^{2}, Q_{3}(\lambda)=\rho^{3} c_{0}$ e $Q_{4}(\lambda)=\rho\left(\rho^{2} c_{1}\right)$, temos que $Q(\lambda)=\left[\left(Q_{1}+Q_{2}+Q_{3}+Q_{4}\right)(\lambda)\right]^{-1}$.

Por [22], se existem os limites $\lim _{\lambda \rightarrow 0} Q_{i}(\lambda) \quad i=1,2,3,4$, então $\lim _{\lambda \rightarrow 0} Q(\lambda)=\left(\lim _{\lambda \rightarrow 0} Q_{1}(\lambda)+\lim _{\lambda \rightarrow 0} Q_{2}(\lambda)+\lim _{\lambda \rightarrow 0} Q_{3}(\lambda)+\lim _{\lambda \rightarrow 0} Q_{4}(\lambda)\right)^{-1}$

Obviamente $\lim _{\lambda \rightarrow 0} Q_{1}(\lambda)=\lim _{\lambda \rightarrow 0} Q_{2}(\lambda)=0$.

Vamos calcular $\lim _{\lambda \rightarrow 0} Q_{3}(\lambda)$ e $\lim _{\lambda \rightarrow 0} Q_{4}(\lambda)$.

b.1) Cálculo de $\lim _{\lambda \rightarrow 0} Q_{3}(\lambda)$

$$
\begin{aligned}
c_{0} & =\frac{1}{\lambda}\left(\mu_{1} c_{11}+\mu_{2} c_{12}+\mu_{3} c_{13}\right) \\
c_{11} & =\frac{1}{\lambda}\left(\left(\lambda+\mu_{1}+\mu_{2}\right) c_{21}-\lambda c_{12}-\mu_{3}\right) \\
& =\frac{1}{\lambda}\left[\left(\lambda+\mu_{1}+\mu_{2}\right)\left(\frac{\mu}{\lambda}-c_{22}-c_{23}\right)-\frac{\lambda \mu_{1}}{\lambda+\mu_{2}}\left(\frac{\mu}{\lambda}+\frac{\left(\mu_{3}-\mu_{1}\right)}{\mu_{1}} c_{22}-c_{23}\right)-\mu_{3}\right] \\
c_{12} & =\frac{\mu_{1} c_{21}+\mu_{3} c_{22}}{\lambda+\mu_{2}} \\
& =\frac{\mu_{1}}{\lambda+\mu_{2}}\left[\frac{\mu}{\lambda}+\frac{\left(\mu_{3}-\mu_{1}\right)}{\mu_{1}} c_{22}-c_{23}\right] \\
& =\frac{1}{\lambda}\left[\frac{\lambda \mu_{1}}{\lambda+\mu_{2}}\left(\frac{\mu}{\lambda}+\frac{\left(\mu_{3}-\mu_{1}\right)}{\mu_{1}} c_{22}-c_{23}\right)\right] \\
c_{13} & =\frac{1}{\lambda}\left[\left(\lambda+\mu_{1}+\mu_{3}\right) c_{23}-\mu_{2}\right]
\end{aligned}
$$

Portanto,

$$
\begin{aligned}
c_{0}= & \frac{1}{\lambda^{2}}\left[\left(\mu_{2}-\mu_{1}\right) \frac{\lambda \mu_{1}}{\lambda+\mu_{2}}\left(\frac{\mu}{\lambda}+\frac{\left(\mu_{3}-\mu_{1}\right)}{\mu_{1}} c_{22}-c_{23}\right)+\mu_{1}\left(\lambda+\mu_{1}+\mu_{2}\right)\left(\frac{\mu}{\lambda}-c_{22}-c_{23}\right)\right. \\
& \left.-\mu_{1} \mu_{3}+\mu_{3}\left(\lambda+\mu_{1}+\mu_{3}\right) c_{23}-\mu_{2} \mu_{3}\right] \\
= & \frac{\left(\mu_{2}-\mu_{1}\right)}{\lambda} \frac{\mu_{1}}{\lambda+\mu_{2}}\left(\frac{\mu}{\lambda}+\frac{\left(\mu_{3}-\mu_{1}\right)}{\mu_{1}} c_{22}-c_{23}\right)+\frac{\mu_{1}}{\lambda^{2}}\left(\lambda+\mu_{1}+\mu_{2}\right)\left(\frac{\mu}{\lambda}-c_{22}-c_{23}\right) \\
& \quad-\frac{\mu_{3}}{\lambda^{2}}\left(\mu_{1}+\mu_{2}\right)+\frac{\mu_{3}}{\lambda^{2}}\left(\lambda+\mu_{1}+\mu_{3}\right) c_{23} \\
\rho^{3} c_{0}= & \frac{\left(\mu_{2}-\mu_{1}\right) \mu_{1}}{\mu^{3}\left(\lambda+\mu_{2}\right)}\left[\lambda \mu+\frac{\left(\mu_{3}-\mu_{1}\right)}{\mu_{1}} \lambda^{2} c_{22}-\lambda^{2} c_{23}\right]+\frac{\mu_{1}\left(\lambda+\mu_{1}+\mu_{2}\right)}{\mu^{3}}\left(\mu-\lambda c_{22}-\lambda c_{23}\right) \\
& \quad-\frac{\mu_{3} \lambda}{\mu^{3}}\left(\mu_{1}+\mu_{2}\right)+\frac{\mu_{3} \lambda}{\mu^{3}}\left(\lambda+\mu_{1}+\mu_{3}\right) c_{23}
\end{aligned}
$$


Portanto,

$$
\lim _{\lambda \rightarrow 0} Q_{3}(\lambda)=\frac{\mu_{1}\left(\mu_{1}+\mu_{2}\right)}{\mu^{3}} \mu=\frac{\mu_{1}\left(\mu_{1}+\mu_{2}\right)}{\mu^{2}}
$$

b.2) Cálculo de $\lim _{\lambda \rightarrow 0} Q_{4}(\lambda)$.

Vamos inicialmente calcular $\lim _{\lambda \rightarrow 0} \rho^{2} c_{1}$.

$$
\begin{aligned}
c_{1} & =\frac{\left(\mu_{1}+\mu_{2}\right) c_{21}+\left(\mu_{2}+\mu_{3}\right) c_{22}+\left(\mu_{1}+\mu_{3}\right) c_{23}}{\lambda} \\
& =\frac{1}{\lambda}\left[\left(\mu_{1}+\mu_{2}\right)\left(c_{2}-c_{22}-c_{23}\right)+\left(\mu_{2}+\mu_{3}\right) c_{22}+\left(\mu_{1}+\mu_{3}\right) c_{23}\right] \\
& =\frac{1}{\lambda}\left[\left(\mu_{1}+\mu_{2}\right) c_{2}+\left(\mu_{3}-\mu_{1}\right) c_{22}+\left(\mu_{3}-\mu_{2}\right) c_{23}\right] \\
\rho^{2} c_{1} & =\frac{\mu_{1}+\mu_{2}}{\mu}+\lambda^{2} \frac{\left(\mu_{3}-\mu_{1}\right)}{\mu^{2}} c_{22}+\lambda^{2} \frac{\left(\mu_{3}-\mu_{2}\right)}{\mu^{2}} c_{23}
\end{aligned}
$$

Como $\exists \lim _{\lambda \rightarrow 0} c_{22}$ e $\exists \lim _{\lambda \rightarrow 0} c_{23}$, temos que $\lim _{\lambda \rightarrow 0} \rho^{2} c_{1}=\frac{\mu_{1}+\mu_{2}}{\mu}$.

Portanto, por [22], $\lim _{\lambda \rightarrow 0} Q_{4}(\lambda)=\lim _{\lambda \rightarrow 0} \rho * \lim _{\lambda \rightarrow 0} \rho^{2} c_{1}=0$

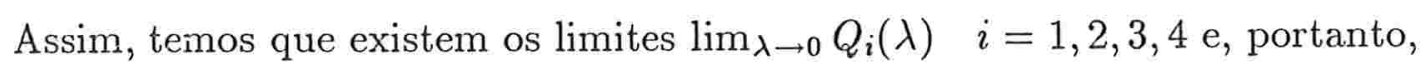

$$
\begin{aligned}
\lim _{\lambda \rightarrow 0} Q(\lambda) & =\left(\lim _{\lambda \rightarrow 0} Q_{1}(\lambda)+\lim _{\lambda \rightarrow 0} Q_{2}(\lambda)+\lim _{\lambda \rightarrow 0} Q_{3}(\lambda)+\lim _{\lambda \rightarrow 0} Q_{4}(\lambda)\right)^{-1} \\
& =\frac{\mu^{2}}{\mu_{1}\left(\mu_{1}+\mu_{2}\right)}
\end{aligned}
$$

Logo, por (A) e (B) temos que

$$
\lim _{\lambda \rightarrow 0} T_{s}^{3}(\lambda)=\frac{1}{\mu} \lim _{\lambda \rightarrow 0} P(\lambda) \lim _{\lambda \rightarrow 0} Q(\lambda)=\frac{1}{\mu} \frac{\mu_{1}+\mu_{2}}{\mu} \frac{\mu^{2}}{\mu_{1}\left(\mu_{1}+\mu_{2}\right)}=\frac{1}{\mu_{1}}
$$

Logo $\lim _{\lambda \rightarrow 0} T_{s}^{3}=\frac{1}{\mu_{1}}$ assume o valor mínimo em $\mu_{1}^{*}=\mu$ e $\mu_{2}^{*}=\mu_{3}^{*}=0$, isto é, com apenas $u m$ servidor.

Portanto temos $T_{3}^{*}(0)=\frac{1}{\mu}$, que é o tempo médio no sistema $M / M / 1$ com carga nula, $S(0, \mu, 1,1)$.

As figuras 4.5 e 4.6 mostram resultados gráficos para a aproximação de $T_{3}^{*}(\lambda)$ para alguns valores de $\mu$.

Na figura 4.5, são traçados os seguintes gráficos para tempo médio no sistema, considerando $\mu=8.0$ : 
- Gráfico para o sistema homogêneo $S(\lambda, 8.0,1,3)$. O tempo médio no sistema neste caso é dado por

$$
\frac{3}{\mu} \frac{2+2 \rho-\rho^{2}}{(1-\rho)\left(3 \rho^{2}+4 \rho+2\right)} \quad \operatorname{com} \quad \rho=\frac{\lambda}{\mu}
$$

- Gráfico para o sistema $S(\lambda, 8.0,1,3)$ com distribuição aproximadamente ótima de capacidades nos servidores. O tempo médio é dado por uma aproximação de $T_{3}^{*}(\lambda)$, uma vez que não foi determinada a distribuição ótima de capacidades dos servidores. $\mathrm{O}$ valor aproximado de $T_{3}^{*}(\lambda)$ foi obtido considerando-se várias possíveis distribuições de capacidades e tomando como distribuição aproximadamente ótima, a que fornece o menor tempo médio no sistema.

- Gráfico para o sistema homogêneo $S(\lambda, 8.0,1,2)$

- Gráfico para o sistema $S(\lambda, 8.0,1,2)$ com distribuição ótima de capacidades.

Podemos observar nesta figura que:

- O sistema de três servidores com distribuição aproximadamente ótima de capacidades tem desempenho pior que o sistema de dois servidores correspondente (mesma capacidade total de atendimento e mesma carga total) com distribuição ótima de capacidades.

- Para cargas baixas no sistema, o sistema de três servidores com distribuição ótima de capacidades tem desempenho melhor que o correspondente sistema homogêneo de dois servidores.

Na figura 4.6, foram traçados os seguintes gráficos:

- Para $\mu=4.0$, os gráficos de tempo médio no sistema $S(\lambda, 4.0,1,2)$ com distribuição ótima de capacidades e de tempo médio no sistema $S(\lambda, 4.0,1,3)$ com distribuição aproximadamente ótima de capacidades.

- Os mesmos gráficos para $\mu=10.0$

Podemos concluir, a partir dos gráficos das figuras 4.5 e 4.6 que:

- A curva que aproxima $T_{3}^{*}(\lambda)$ tem um ponto de inflexão para um valor $\lambda=\bar{\lambda}$, dependente de $\mu$, sendo a função côncava até $\bar{\lambda}$ e convexa a partir de $\bar{\lambda}$. 
- O sistema de três servidores com distribuição aproximadamente ótima tem desempenho pior que o sistema de dois servidores com distribuição ótima de capacidades. 
II

FIG. $\quad 4.5$ 
$\int_{3}^{2}$ FIG. 4.6

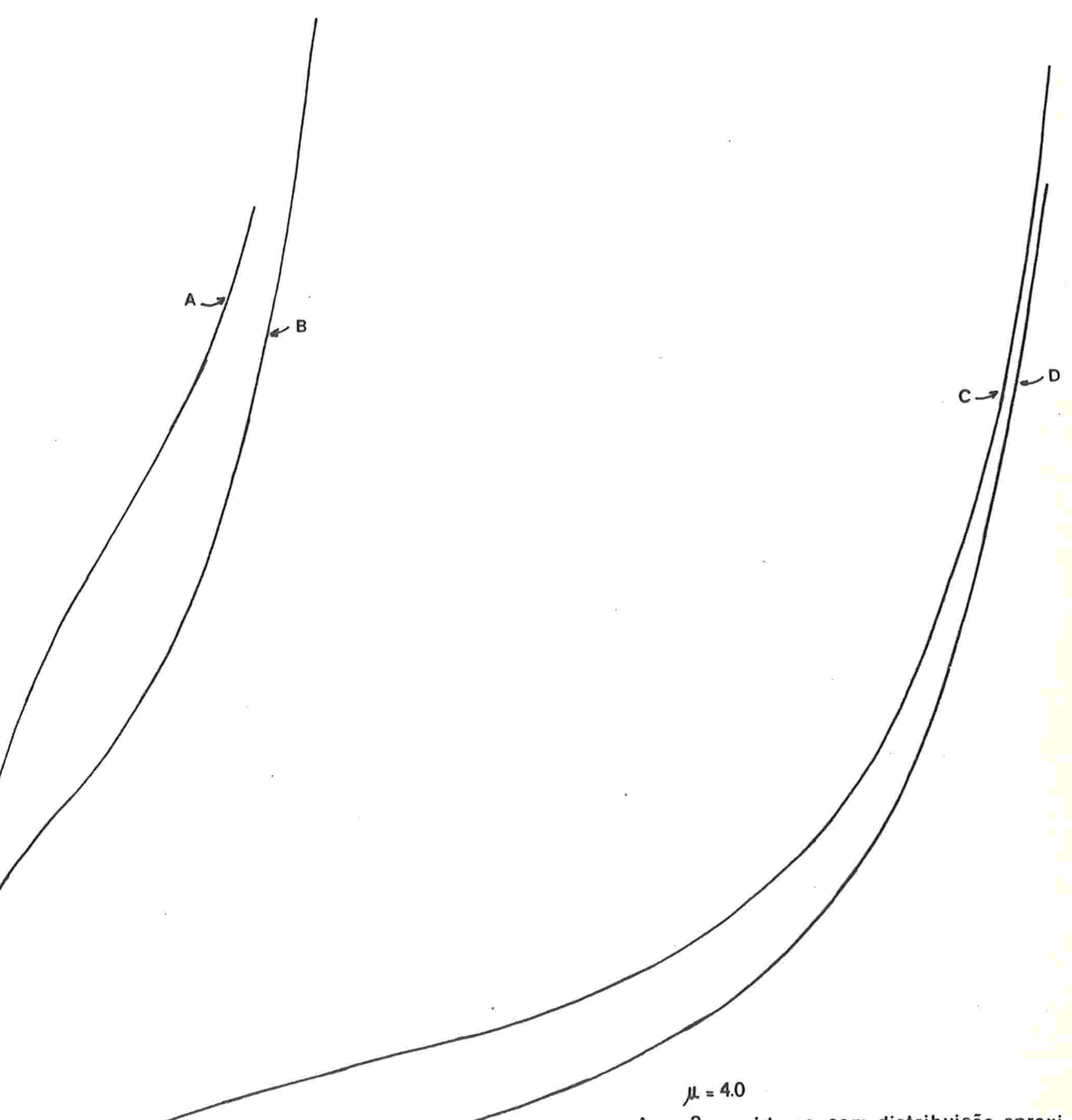

9

A - 3 servidores com distribuição aproxi madamente ótima de capacidades

B - 2 servidores com distribuiçäo ótima de capacidades

$$
\mu=10.0
$$

c - 3 servidores com distribuição aproximadamente ótima de capacidades D - 2 servidores com distribuição ótima de capacidades 


\subsubsection{Análise para tempo de fila}

Seja $N_{f}^{3}$ - número médio de jobs na fila do sistema de três servidores

$$
\begin{aligned}
N_{f}^{3} & =1 * p_{4}+2 * p_{5}+\cdots \\
& =\sum_{j=4}^{\infty}(j-3) * p_{j} \quad \text { com } p_{j}=c_{j} * p_{3}=\rho^{j-3} p_{3} \\
& =\left(\sum_{i=1}^{\infty} i \rho^{i}\right) * p_{3}=\frac{\rho}{(1-\rho)^{2}} * p_{3}
\end{aligned}
$$

E, por Little, obtemos o tempo médio na fila do sistema com três servidores

$$
T_{f}^{3}=\frac{1}{\lambda} \frac{\rho}{(1-\rho)^{2}} p_{3}
$$

É fácil verificar que para $\mu_{1}=\mu_{2}=\mu_{3}$ a fórmula acima fornece a conhecida expressão para tempo médio na fila do sistema $M / M / 3$ com servidores iguais.

A expressão para tempo médio na fila do sistema $M / M / 3$ com servidores iguais é dada por (vide [16], [30])

$$
T_{H}^{3}=\frac{(3 \rho)^{3} P_{0}}{3 ! \mu(1-\rho)^{2}} \quad \text { onde } \quad P_{0}=\left[\frac{(3 \rho)^{3}}{3 !(1-\rho)}+1+3 \rho+\frac{(3 \rho)^{2}}{2 !}\right]^{-1}=\frac{2(1-\rho)}{2+4 \rho+3 \rho^{2}}
$$

Portanto,

$$
T_{H}^{3}=\frac{9 \rho^{3}}{2 \mu(1-\rho)^{2}} \frac{2(1-\rho)}{\left(3 \rho^{2}+4 \rho+2\right)}=\frac{9 \rho^{3}}{\mu(1-\rho)\left(3 \rho^{2}+4 \rho+2\right)}
$$

Mas para $\mu_{1}=\mu_{2}=\mu_{3}=\frac{\mu}{3}$, conforme mostrado para tempo no sistema, vale que $p_{3}=\frac{9 \rho^{3}(1-\rho)}{3 \rho^{2}+4 \rho+2}$

Logo

$$
T_{f}^{3}=\frac{1}{\lambda} \frac{\rho}{(1-\rho)^{2}} \frac{9 \rho^{3}(1-\rho)}{\left(3 \rho^{2}+4 \rho+2\right)}=\frac{9 \rho^{3}}{\mu(1-\rho)\left(3 \rho^{2}+4 \rho+2\right)}
$$




\section{Análise do comportamento de $T_{f}^{*}$ como função de $\lambda$}

O objetivo dessa análise é verificar como a função que determina o menor tempo médio na fila do sistema $S(\lambda, \mu, 1,3)$ se comporta com a variação da carga no sistema. Vamos denotar por $T_{f}^{*}(\lambda)$ ao valor ótimo para tempo médio na fila do sistema $S(\lambda, \mu, 1,3)$.

As figuras 4.7 e 4.8 mostram resultados gráficos para a aproximação de $T_{f}^{*}(\lambda)$ para alguns valores de $\mu$.

Na figura 4.7 foram traçados os seguintes gráficos para tempo médio na fila do sistema, considerando $\mu=8.0$ :

- Gráfico para o sistema $S(\lambda, 8.0,1,3)$ com distribuição aproximadamente ótima de capacidades nos servidores. O tempo médio na fila do sistema é dado por uma aproximação de $T_{f}^{*}(\lambda)$, uma vez que não foi determinada a distribuição ótima de capacidades nos servidores. $\mathrm{O}$ valor aproximado de $T_{f}^{*}(\lambda)$ foi obtido considerando-se várias possíveis distribuições de capacidades e tomando como distribuição aproximadamente ótima a que fornece o menor tempo médio na fila do sistema.

- Gráfico para o sistema $S(\lambda, 8.0,1,2)$ com distribuição ótima de capacidades.

- O gráfico para o sistema $S(\lambda, 8.0,1,3)$ homogêneo foi omitido pois os valores para tempo médio na fila do sistema homogêneo são muito próximos aos do sistema heterogêneo aproximadamente ótimo, e portanto difíceis de serem representados na escala escolhida.

Na figura 4.8 foram traçados os seguintes gráficos:

- Para $\mu=4.0$, os gráficos de tempo médio na fila do sistema $S(\lambda, 4.0,1,2)$ com distribuição ótima de capacidades e tempo médio na fila do sistema $S(\lambda, 4.0,1,3) \mathrm{com}$ distribuição aproximadamente ótima de capacidades.

- Os mesmos gráficos para $\mu=10.0$.

Podemos concluir a partir dos gráficos das figuras 4.7 e 4.8 que:

- O sistema de três servidores com distribuição aproximadamente ótima de capacidades tem desempenho melhor que o correspondente sistema de dois servidores com distribuição ótima de capacidades.

- A função $T_{f}^{*}$ é convexa em $\lambda$. 
FIG. 4.7

$$
\mu=8.0
$$

A-3 servidores com distribuição aproximadamente ótima de capacidades

B-2 servidores com distribuição ótima de capacidades

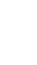

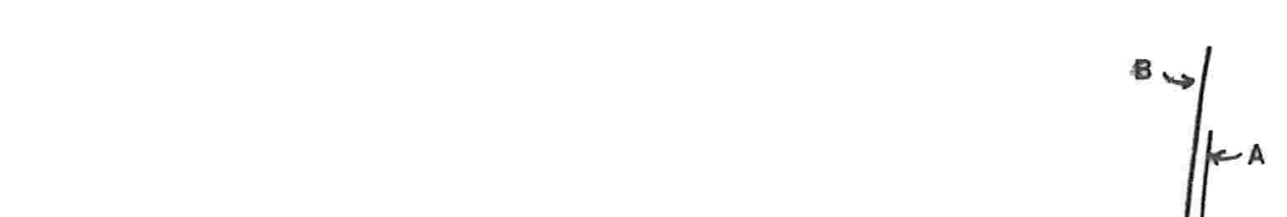


FIG. 4.8
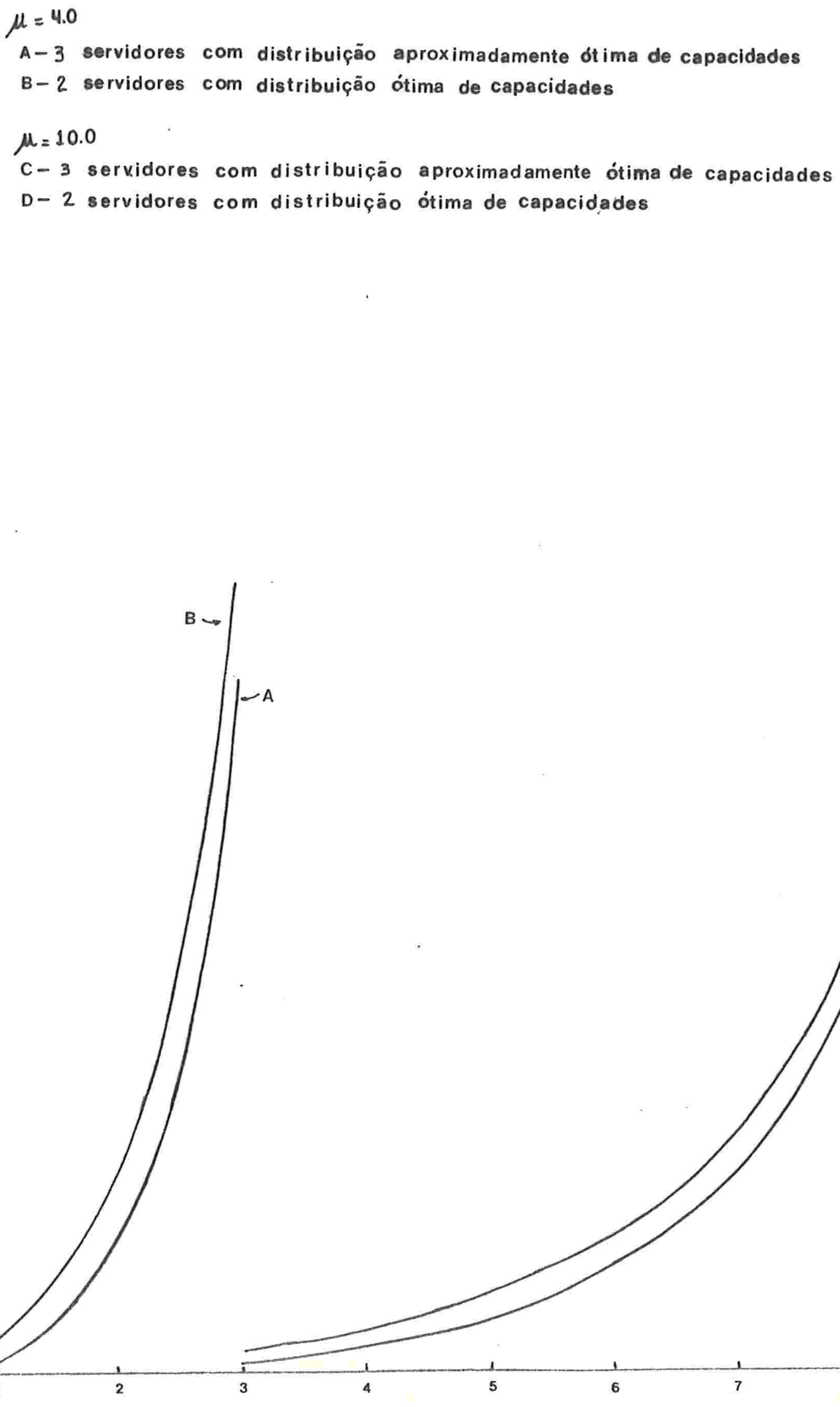


\subsubsection{Conclusão para três servidores}

No estudo do comportamento de um sistema $S(\lambda, \mu, 1,3)$, com servidores com capacidades não necessariamente homogêneas, não foram obtidas de forma exata as distribuições ótimas de capacidades entre os servidores, com relação a tempo médio no sistema e a tempo médio na fila do sistema, pois as expressões obtidas para cálculo desses tempos são difíceis de serem manipuladas. Assim, foram obtidos valores aproximdados para essas distribuições ótimas. Pudemos comprovar, graficamente, o seguinte resultado relativo ao desempenho aproximadamente ótimo do sistema:

- Considerando-se a medida tempo médio no sistema, o desempenho aproximadamente ótimo do sistema com três servidores é pior que o desempenho ótimo do correspondente sistema com dois servidores.

- Considerando-se a medida tempo médio na fila do sistema, o desempenho aproximadamente ótimo do sistema com três servidores é melhor que o desempenho ótimo do correspondente sistema com dois servidores.

\subsection{Conclusão}

Este capitulo dedicou-se principalmente ao estudo do desempenho de grupos de $\mathrm{m}$ servidores, chamados grupos não singulares, que são sistemas de filas $M / M / m$ não homogêneos.

O estudo detalhado do caso de dois servidores levou à obtenção da distribuição ótima de capacidades. Essa distribuição ótima confere com a descrita em [20], embora tenha sido obtida por caminhos diferentes. Quanto ao desempenho ótimo, foi mostrado que em relação a tempo médio no sistema, o sistema de dois servidores é pior que o sistema de um servidor correspondente e que em relação a tempo médio na fila do sistema, o sistema de dois servidores é melhor que o sistema de um servidor correspondente.

O estudo do caso de três servidores se limitou à obtenção das expressões de tempo médio no sistema e de tempo médio na fila do sistema. Estas expressões não são conhecidas na literatura a que se teve acesso, mas também não são de simples manuseio. Quanto ao desempenho, foram consideradas aproximações e gráficos que permitiram concluir que: com 
relação a tempo médio no sistema, o sistema de três servidores com distribuição aproximadamente ótima de capacidades é pior que o sistema de dois servidores correspondente com distribuição ótima de capacidades e com relação a tempo médio na fila do sistema, o sistema de três servidores com distribuição aproximadamente ótima de capacidades é melhor que o sistema de dois servidores correspondente com distribuição ótima de capacidades.

Estes resultados, obtidos para dois e três servidores, vem de acordo com a intuição e também com os resultados existentes para sistemas $M / M / m$ homogêneos ([16], [14]).

Assim, pode-se partir para uma conjetura relativa ao desempenho ótimo do sistema $M / M / m$ não homogêneo:

- Com relação ao tempo médio no sistema, o desempenho ótimo degrada à medida que $\mathrm{m}$ cresce.

- Com relação a tempo médio na fila dos sistema, o desempenho ótimo melhora à medida que $m$ cresce.

Levando-se em consideração que o tempo médio no sistema é mais efetivo como medida de desempenho, uma vez que considera o tempo total do job no sistema, pode-se dizer que o desempenho do sistema melhora com a concentração da capacidade de atendimento (utilização de servidores mais rápidos). Assim, a conjetura se resume na utilização do 'superserver' para otimização de desempenho.

Existe um resultado bastante interessante em [25], que diz que um sistema $S(\lambda, \mu, 1, m)$ com servidores homogêneos e/ou com servidores inabilitados (capacidade de atendimento igual a zero) nunca tem desempenho ótimo, com relação a tempo médio no sistema (este resultado foi demonstrado neste capítulo para o caso de $m=2$ ). Este resultado é compativel com a conjetura e ainda mostra que os sistemas homogêneos, tão utilizados na literatura, nunca são ótimos.

Portanto, um sistema cuja estratégia de load sharing é a partição de servidores, tem melhor desempenho, em relação a tempo médio no sistema, com o 'superserver global' (centralização). Isto porque este capítulo levou à utilização do 'superserver' ao invés de partições não singulares e o capítulo anterior mostrou a superioridade do 'superserver global' em relação a várias partições singulares.

Vale lembrar que a centralização de atendimento acarreta problemas que não foram discutidos neste trabalho, envolvidos por exemplo com 'gargalo' de comunicação ou com 
custos associados à capacidade de atendimento de servidores. 


\section{Capítulo 5}

\section{Conclusão}

O capítulo 2 é essencialmente didático e introduziu, fundamentado em [29], a maioria dos conceitos necessários para o desenvolvimento do trabalho. Além disso, existe uma preocupação quanto à análise crítica da medida de desempenho introduzida neste artigo básico.

Os capítulos 3 e 4 dedicaram-se à análise de otimalidade de um sistema que se utiliza da estratégia de 'load sharing' estática denominada 'partição de servidores'. Esta análise considerou proporcionalidade (linearidade) entre custos e capacidades de atendimento dos servidores.

No capítulo 3, a análise de otimalidade foi feita em relação a partições singulares sob os enfoques de projeto $((\lambda, \mu))$-problema), de avaliação e ajuste de desempenho ( $\lambda$ problema) e de possiveis evoluções ( $\mu$-problema) do sistema. Neste capítulo foram obtidos dois resultados muito interessantes. O primeiro definiu a alocação ótima de carga nas origens em função de intervalos da carga total, quando os servidores têm capacidade de atendimento decrescentes. Este resultado mostrou que existem cargas de desativação para os servidores $m,(m-1), \cdots, 2$, que originam $m$ intervalos de carga, onde no intervalo de carga mais alta os m servidores estão ativos na alocação ótima, no intervalo de carga imediatamente inferior os (m-1) servidores mais 'fortes' estão ativos na alocação ótima (o 
m-ésimo servidor é desativado), e assim por diante, até que no intervalo de carga mais baixa somente o servidor mais 'forte' está ativo na alocação ótima (o primeiro servidor). O segundo resultado mostra a otimalidade da concentração de capacidade de atendimento em um só servidor, com decorrente concentração da carga total na origem associada. Este resultado significa na verdade que, sob as hipóteses consideradas, o melhor desempenho é obtido com a partição formada por apenas um grupo singular.

Já no capítulo 4 houve a preocupação em aprofundar a análise da 'partição de servidores', considerando basicamente grupos não singulares de uma partição, o que corresponde a analisar sistemas de filas $M / M / m$ com servidores heterogêneos (servidores com capacidade de atendimento distintas). Neste capítulo obtivemos, para o caso de dois servidores, independentemente de Nishida ([20]), as expressões para tempo médio no sistema e para alocação ótima de capacidades dos servidores, considerando a escolha do servidor mais rápido sempre que possível (FSR - 'fastest service rule'). Foram também obtidas expressões para tempo médio no sistema para duas possivieis generalizações do problema: a primeira que considera que um job pode ser ou não aceito pelo sistema ('balking') e a segunda que considera uma distribuição de probabilidade associada à alocação dos jobs aos servidores (o job é enviado ao servidor i com probabilidade $p_{i}$ ). A partir dessa segunda generalização foi possível mostrar que a utilização da regra FSR é a melhor alternativa para esse sistema. Para o caso de três servidores, obtivemos somente a expressão para tempo médio no sistema, que não tem uma forma facilmente tratável. Desse estudo surgiu a conjectura de que a concentração da capacidade de atendimento, isto é, a utilização do grupo singular correspondente ao grupo não singular em questão, fornece o 'melhor' desempenho. Além disso, esse estudo trouxe o resultado da não otimalidade de um sistema de fila homogêneo, que é um sistema bastante usual na literatura sobre sistemas de filas.

Assim, a análise feita nesses dois capítulos sugeriu a 'otimalidade do superserver', que vem a ser um servidor único com concentração total da capacidade de atendimento.

Existem muitas possíveis variações no estudo de otimalidade da 'partição de servidores', que podem ser bastante interessantes. Entre elas, podemos citar:

- Introdução da não proporcionalidade entre custos e capacidades de atendimento dos servidores;

- Agrupamento de servidores em grupos com funções diferenciadas, considerando que 
os jobs são classificados por função (ver [10]).

Devido à ampla abordagem teórica apresentada em [29], em relação à modelagem de sistemas distribuídos, existe uma abertura muito grande de trabalho, onde podemos incluir:

- A introdução do efeito do 'overhead' de comunicação, sendo que este 'overhead' pode ser considerado sob dois pontos de vista, dependendo da estratégia de 'load sharing' a ser analisada:

- O primeiro, devido à comunicação inter-programas, que pressupõe uma avaliação da quantidade de informação trocada entre programas (ver [02], [03], [24] e [11]);

- E o segundo, devido à 'transferência de jobs' no sistema, que consiste do tempo necessário para job 'viajar' da sua origem (entrada) até seu servidor (destino) (ver [26]);

- O estudo das estratégias dinâmicas, que apresentam um grau de dificuldade maior que o das estratégias estáticas (ver [19]);

- O estudo do efeito nos algoritmos canônicos de algumas das variações existentes na área de controle ótimo de filas. Entre essas variações podemos citar:

- No controle do processo de chegada de um job no sistema, ele pode ser aceito ou não no sistema. Em [20], foi considerada essa variação para o estudo de sistemas $M / M / 2$ heterogêneos e otimalidade significou minimização de jobs perdidos. Os resultados obtidos para alocação ótima de capacidades foram similares aos estudados no capítulo 4;

- No controle da disciplina de atendimento na fila pode-se considerar prioridades para atendimento, sendo que cada job que entra no sistema tem uma prioridade associada (ver [16], onde são considerados alguns tipos interessantes de prioridade para aplicação em sistemas de computadores).

Desta lista parcial de referências brevemente comentadas vemos que o escopo de possibilidades de pesquisa é imenso, além das questões que surgem naturalmente de respostas parciais dadas nesta tese, como por exemplo, a obtenção de expressões para tempo médio no sistema e para alocação ótima de capacidades para sistemas $M / M / m$ heterogêneos $(m \geq 4)$.

A relevância da questão é grande e esperamos ter contribuido para que a ponte entre 
otimização e projeto de sistemas distribúdos tenha sido aumentada. Tal esperança está associada a nossa crença da validade do ferramental apresentado para a classe de problemas em questão. 


\section{BIBLIOGRAFIA}

[01] AGRAWALA, A.K. \& TRIPATHI, S.K. On the optimality of semidynamic routing schemes. Information Processing Letters, 13(1):20-22, 1981.

[02] CHOW, T.C.K. \& ABRAHAN, J.A. Load balancing in distributed systems. IEEE Transactions on Software Engineering, 8(4):401-412, 1982.

[03] CHU, W. Task allocation in distributed data processing. Computer, 13(11): 57-69, 1980.

[04] CRABILL, T.B.; GROSS, D.; MAGAZINE, M.J. A classified bibliography of research on optimal design and control of queues. Operations Research, 25(2):219-232, 1977.

[05] DANTAS, C.A.B., et al. Tópicos de filas e simulaçâo. São Paulo, IME-USP, 1978. 75p.

[06] DANTAS, C.A.B. \& RODRIGUES, F.W. Tópicos de processos estocásticos. Rio de Janeiro, IMPA, 1977. 223p.

[07] FELLER, W. Introduction to probability theory and its applications. 2.ed. New York, John Wiley, 1961/1971, 2v.

[08] FOSCHINI, G.J. On heavy traffic diffusion analysis and dynamic routing in packet switched networks. In: CHANDY, K.M. \& REISER, M. eds. Computer Performance. Amsterdam, North-Holland, 1977.

[09] GEOFFRION, A.M. Elements of large-scale mathematical programming. In: Perspectives on optimization: a collection of expository articles. Reading, Addison-Wesley, c1972. p.25-64. 
[10] GLICKMANN, T.S. Resource allocation to minimize delay in a dual-purpose service facility. Operational Research Quarterly, 26(2):305-315, 1975.

[11] GYLYS, V.B. \& EDWARDS, J.A. Optimal partitioning of workload for distributed systems. Digest of Papers COMPCON, Fall 76, sep. 1976, p.353357.

[12] HUMES JR., C. Alguns aspectos de dualidade em programação matemática. São Paulo, 1972. 165p. Dissertação (Mestrado) - EPUSP.

[13] HUMES JR., C. Problemas de grande porte em programação matemática. In: REU NIÃO DE MATEMÁTICA, 1, Rio de Janeiro, IBM, 1978. p.1-80.

[14] HUMES JR., C. Tópico de otimização e redes de computadores. São Paulo, 1988. 109p. Tese (Livre Docência) - IME-USP.

[15] KLEINROCK, L. Communication nets: stochastic message flow and delay. New York, Dover, 1972. 209p.

[16] KLEINROCK, L. Queueing systems. New York, John Wiley, 1975-76, 2v.

[17] MANGASARIAN, O.L. Nonlinear programming. New York, McGraw Hill, 1969. 220p. (McGraw-Hill series in systems science)

[18] MORRIS, R.J.T. \& WANG, Y.T. Some results for multi-queue systems with multiple cyclic servers. In: RUDIN, H. \& BUX, W., eds. Performance of computer-communication systems. Elsevier, 1984. p.245-258.

[19] NI, L.M. \& ABANI, K. Nonpreemptive load balancing in a class of local area networks. In: Computer Networking Symposium. Proceedings. Gaithersburg, 1981. New York, IEEE, 1981. p.113-118.

[20] NISHIDA, T.; TAHARA, A.; HANAI, H. Optimal design for heterogeneous two-server queue. Osaka, Osaka University, 1972. p.295-301 (TR 1053) 
[21] REIMAN, M.I. \& SIMON, B. Queues in light traffic. (a ser publicado)

[22] RUDIN, W. Principles of mathematical analysis. New York, McGraw-Hill, 1964. 270p. (International Series in Pure and Applied Mathematics)

[23] SINGH, V.P. Two-server markovian queues with balking: heterogeneous vs. homogeneous servers. Operations Research, 18(1):145-159, 1970.

[24] STONE, H.S. \& BOKHARI, S.H. Control of distributed process. Computer, 11(7):97-106, 1978.

[25] TAHARA, A. \& NISHIDA, T. Optimal allocation of service rates for multiserver markovian queue. Journal of Operations Research Society of Japan, 18(1/2):90-96, 1975.

[26] TANTAWI, A.N. \& TOWSLEY, D. Optimal static load balancing in distributed computer systems. Journal of the Association for Computing Machinery, 32(2):445-465, 1985.

[27] TRIVEDI, K.S. \& HARING, G. Load distribution in a star configured system with error-prone channels. Matemática Aplicada e Computacional, $3(1): 23-40,1984$.

[28] VARAYA, P.P. Notes on optimization. New York, Van Nostrand, c1972. 202p. (Van Nostrand Reinhold Notes on Systems Sciences)

[29] WANG, Y.T. \& MORRIS, R.J.T. Load sharing in distributed systems. IEEE Transactions on Computers, 34(3):204-217, 1985.

[30] WHITE, J.A.; SCHMIDT, J.W.; BENNETT, G.K. Analysis of queueing systems. New York, Academic Press, 1975. 532p.

[31] WHITT, W. The queueing network analyzer. The Bell System Technical Journal, 62(9):2779-2815, 1983. 
Página

Capítulo 1 - Introdução

Capítulo 2 - Análise de Desempenho de Sistemas Distribuídos $\quad$..................... 3

2.1 - Apresentação de sistemas distribuídos $\quad$....................................... 4

2.2 - Load Sharing $\quad$............................................................... 8

2.2.1 - Apresentação das estratégias de load sharing canônicas $\quad$... 10

2.2 .2 - Análise das estratégias canônicas $\quad$................................... 14

2.2.2.1 - Análise com carga homogênea $\quad$........................... 20

2.2.2.2 - Análise com carga não homogênea $\quad$.................... 24

2.2.2.3 - Comentários adicionais $\quad$.................................... 26

2.3 - Conclusão $\quad$............................................................................. 29

Capítulo 3 - Análise do Algoritmo Partição dos Servidores $\quad$.......................... 31

3.1 - Formulação do algoritmo $\quad$................................................. 32

3.2 - Análise do algoritmo para $\mathrm{N}=\mathrm{K} \quad$................................. 36

3.2 .1 - Análise do $\lambda$-problema $\quad$............................................ 37

3.2.1.1 - Análise para $\mathrm{N}=\mathrm{K}=2 \quad$.......................... 37

3.2.1.2 - Análise para $\mathrm{N}=\mathrm{K}=\mathrm{m} \quad$.......................... 48

3.2 .2 - Análise do $\mu$-problema $\quad$.............................................. 63

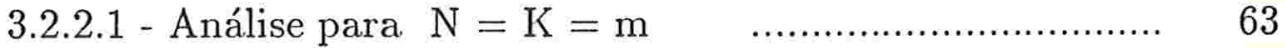

3.2.3 - Alocação de serviço X alocação de capacidades:

Comparação entre o $\lambda$-problema e o $\mu$-problema ................... 67

3.2.4 - Análise do $(\lambda, \mu)$-problema $\quad$.................................... 69

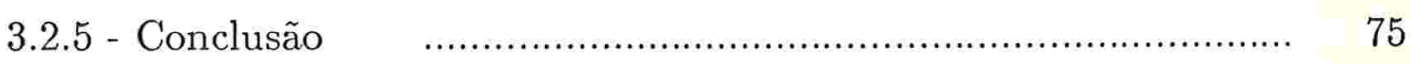


Capítulo 4 - Partição Não Singular de Servidores

4.1 - Especificação completa do grupo não singular $\quad$........................... 79

4.2 - Grupo de dois servidores $\quad$.................................................... 81

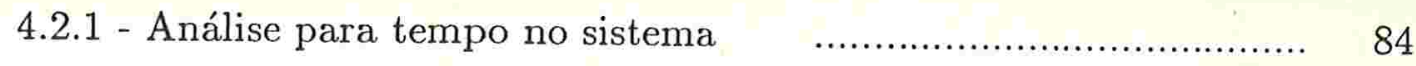

4.2 .2 - Análise para tempo de fila $\quad$.......................................... 102

4.2 .3 - Conclusão para dois servidores $\quad$..................................... 106

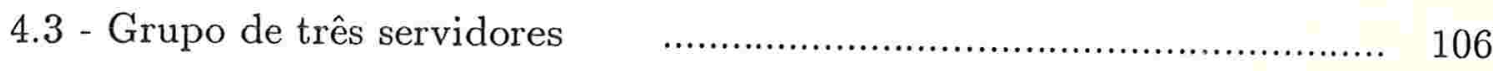

4.3.1 - Análise para tempo no sistema $\quad$.................................... 111

4.3.2 - Análise para tempo de fila $\quad$......................................... 118

4.3 .3 - Conclusão para três servidores $\quad$ …................................. 120

4.4 - Conclusão $\quad$................................................................... 120

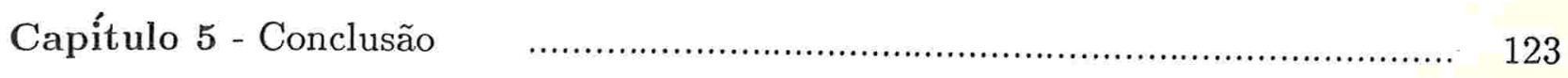

\section{Bibliografia}

